Draft Version MARCh 19, 2019

Typeset using $\mathrm{IAT}_{\mathrm{E}} \mathrm{X}$ modern style in AASTeX61

\title{
A COMPREHENSIVE ANALYSIS OF SPITZER SUPERNOVAE
}

\author{
Tamás Szalai, ${ }^{1,2}$ Szanna Zsíros, ${ }^{1}$ Ori D. Fox, ${ }^{3}$ Ondřej Pejcha, ${ }^{4,5}$ And \\ TOMÁs MÜLLER ${ }^{6,7,8,9}$
}

${ }^{1}$ Department of Optics and Quantum Electronics, University of Szeged, H-6720 Szeged, Dóm tér 9., Hungary

${ }^{2}$ Konkoly Observatory, MTA CSFK, Konkoly-Thege M. út 15-17, Budapest, 1121, Hungary

${ }^{3}$ Space Telescope Science Institute, 3700 San Martin Drive, Baltimore, MD 21218, USA

${ }^{4}$ Institute of Theoretical Physics, Faculty of Mathematics and Physics, Charles University in Prague, Czech Republic

${ }^{5}$ Lyman Spitzer Jr. Fellow, Department of Astrophysical Sciences, Princeton University, 4 Ivy Lane, Princeton, NJ 08540, USA

${ }^{6}$ Millennium Institute of Astrophysics, Santiago, Chile

${ }^{7}$ Instituto de Astrofísica, Pontificia Universidad Católica de Chile, Av. Vicuña Mackenna 4860, 782-0436 Macul, Santiago, Chile

${ }^{8}$ Department of Physics and Astronomy, University of Southampton, Southampton, Hampshire, SO17 $1 B J, U K$

${ }^{9}$ LSSTC Data Science Fellow

\author{
(Received ...; Revised ...; Accepted ......) \\ Submitted to ApJ
}

\begin{abstract}
The mid-infrared (mid-IR) wavelength regime offers several advantages for following the late-time evolution of supernovae (SNe). First, the peaks of the SN spectral energy distributions shift toward longer wavelengths following the photospheric phase. Second, mid-IR observations suffer less from effects of interstellar extinction. Third, and perhaps most important, the mid-IR traces dust formation and circumstellar interaction at late-times ( $>100$ days) after the radioactive ejecta component fades. The Spitzer Space Telescope has provided substantial mid-IR observations of SNe since its launch in 2003. More than 200 SNe have been targeted, but there are even more SNe that have been observed serendipitously. Here we present the results of a comprehensive study based on archival Spitzer/IRAC images of more than 1100 SN positions; from this sample, 119 SNe of various subclasses have been detected,
\end{abstract}

Corresponding author: Tamás Szalai szaszi@titan.physx.u-szeged.hu 
including $45 \mathrm{SNe}$ with previously unpublished mid-IR photometry. The photometry reveal significant amounts of warm dust in some cases. We perform an in-depth analysis to constrain the origin and heating mechanism of the dust, and present the resulting statistics.

Keywords: supernovae: general - infrared: stars - circumstellar matter 


\section{INTRODUCTION}

Tracing the multi-wavelength evolution of supernovae (SNe) over many years, and even decades, can provide important clues about the shock physics, circumstellar environment, and dust production. The current ground-based transient surveys ensure the optical follow-up of hundreds of SNe per year, but these observations are typically at early times during the photospheric phase. Late-time optical spectra and/or non-optical observations are more rare because they require large apertures or space telescopes.

The Spitzer Space Telescope (hereafter Spitzer) has been the primary source of midinfrared (mid-IR) observations of many SNe. Between 2003 and 2009, in the cryogenic (or Cold Mission) phase, only a moderate number $(<50)$ of nearby SNe were targeted by Spitzer. Since 2009, even with post-cryo (Warm Mission) Spitzer, more than 150 more SNe have been targeted. Two surveys, in particular, contributed to this surge: a program aimed to observe a large sample of Type IIn SNe (73 observed SN sites, 13 detected targets, see Fox et al. 2011, 2013), and SPitzer InfraRed Intensive Transients Survey (SPIRITS), a systematic mid-IR study of nearby galaxies (Kasliwal et al. 2017). SPIRITS has resulted in the detection of 44 objects of various types of SNe (observing 141 sites, Tinyanont et al. 2016), three obscured SNe missed by previous optical surveys (Jencson et al. 2017, 2018), and a large number of other variables and transients including ones with unusual infrared behavior (Kasliwal et al. 2017).

These mid-IR observations have several advantages over optical observations, including increased sensitivity to the ejecta as it expands and cools, less impact by interstellar extinction, and coverage of atomic and molecular emission lines generated by shocked gas as it cools (see e.g. Reach et al. 2006). Most of the mid-IR observations are sensitive to warm dust in the SN environment. The origin and heating mechanism of the dust, however, is not always obvious as the dust may be newly formed or pre-existing in the circumstellar medium (CSM). Newly-condensed dust may form in either the ejecta or in a cool dense shell (CDS) produced by the interaction of the ejecta forward shock with a dense shell of CSM (see e.g Pozzo et al. 2004; Mattila et al. 2008; Smith et al. 2009). Pre-existing dust may be radiatively heated by the peak SN luminosity or by X-rays generated by late-time CSM interaction, thereby forming an IR echo (see e.g. Bode \& Evans 1980; Dwek 1983; Graham \& Meikle 1986; Sugerman 2003; Kotak et al. 2009). In this case, the dust is a useful probe of the CSM characteristics and the pre-SN mass loss from either the progenitor or companion star (see e.g. Gall et al. 2011, for a review).

Based on theoretical expectations (see e.g. Kozasa et al. 2009; Gall et al. 2011), Type II-P explosions are likely the best candidates for dust formation among SNe. Some of these objects were targets of Spitzer observations in the early years of the mission. These data typically trace dust formation $\sim 1-3$ yr after explosion and estimate the physical parameters of newly-formed dust. In addition to several detailed studies of single objects (e.g. Meikle et al. 2006, 2007, 2011; Sugerman et al. 
2006; Kotak et al. 2009; Andrews et al. 2010; Fabbri et al. 2011; Szalai et al. 2011), Szalai \& Vinkó (2013) presented an analysis of twelve Type II-P SNe, yielding nine detections and three upper limits. The results do not support the theoretical prediction of significant $\left(>>0.001 M_{\odot}\right)$ dust production in SNe or the large dust masses observed in some old SN remnants and/or high-redshift galaxies. Several ways to reconcile this inconsistency include imperfections of grain condensation models, the probability of clumping dust formation, or significant grain growth in the interstellar matter (ISM) (see Gall et al. (2011) for a review, as well as Szalai \& Vinkó (2013)). Another possibility is that a significant amount of dust may be present in the form of very cold $(<50 \mathrm{~K})$ grains in the ejecta, but to date, far-IR and sub-mm observations have only been able to detect such dust in the very nearby case of SN 1987A (Matsuura et al. 2011, 2015; Indebetouw et al. 2014; Wesson et al. 2015).

Type IIn SNe exhibit signatures of interaction between the ejecta and dense CSM. This shock interaction may lead to either heating of pre-existing circumstellar grains or dust condensation in the CDS that can form between the forward and reverse shock. Papers on individual objects (e.g. Gerardy et al. 2002; Fox et al. 2010; Andrews et al. 2011a; Gall et al. 2014), together with the comprehensive Spitzer study of SNe IIn mentioned above (Fox et al. 2011, 2013) show how the mid-IR evolution can be used to the trace the mass-loss history of the progenitor in the years leading up to the SN.

In contrast with the relatively large number of Type II-P and IIn SNe with published Spitzer data, there are fewer published mid-IR observations of thermonuclear explosions of C/O white dwarfs (Type Ia SNe) or stripped-envelope core-collapse SNe (SE CCSNe, including Type Ib/c, Ibn, and IIb ones). Historically, these SN subclasses are less likely to form new dust due to their high ejecta velocities and less likely to have pre-existing, dense CSMs. For example, Chomiuk et al. (2016) and Maeda et al. (2015a) use radio and near-IR observations, respectively, to place strict upper limits on the amount of material surrounding SNe Ia.

In recent years, however, many SNe within the SNe Ia and stripped-envelope subclasses have shown signs of a dense CSM and/or warm dust. For example, SNe IaCSM, which are thought to be thermonuclear explosions exploding in dense, H-rich shells of ambient CSM (producing IIn-like emission features in their late-time spectra, see e.g. Silverman et al. 2013; Fox et al. 2015; Inserra et al. 2016) and are very bright in mid-IR, even 3-4 years after explosion (Fox \& Filippenko 2013; Graham et al. 2017). The subluminous thermonuclear Type Iax SN 2014dt showed an excess of mid-IR emission (over the expected fluxes of more normal SNe Ia) at $\sim 1$ yr after explosion (Fox et al. 2016, see also in Section 3.2), and an excess of near-IR emission was observed by circumstellar dust around the super-Chandrasekhar candidate SN 2012dn (Yamanaka et al. 2016; Nagao et al. 2017).

Some stripped-enveloped SNe show mid-IR emission at late-times, too. For example, the Type Ic SN 2014C showed a excess of mid-IR emission develop $\sim 1$ year post-explosion (Tinyanont et al. 2016), as did several SNe IIb, including SN 2013df 
(Szalai et al. 2016; Tinyanont et al. 2016) and SN 2011dh (Helou et al. 2013). The Type Ibn SN subclass, which shows narrow helium lines, do not typically show a late-time mid-IR excess (respect to the expected flux level originates from the cooling ejecta). However, the Type Ibn SN 2006jc was bright in early-time Spitzer images (Mattila et al. 2008).

Despite the relatively high number of SNe with reported Spitzer observations, most of the analysis consists of single object papers. There have been some broader studies on SNe IIn (Fox et al. 2011, 2013), SNe IIP (Szalai \& Vinkó 2013), and SNe Ia (Johansson et al. 2017), and a SPIRITS summary by Tinyanont et al. (2016), which includes observations of $\sim 140$ core-collapse SNe within $20 \mathrm{Mpc}$.

The motivation of the current work, however, is to provide a complete review of all SNe currently in the Spitzer archive to compare the mid-IR properties of different SN subclasses. This paper includes mid-IR observations of more than 1100 SN positions, from which 119 objects have been detected. Within this detected sample, many observations were previously unpublished and 45 targets were observed serendipitously during other science programs.

In Section 2, we describe the steps of data collection and photometry of Spitzer/IRAC (Infrared Array Camera) data. We present our results in Section 3, including a statistical analysis of the mid-IR evolution of the different SN subclasses and simple models fits to the spectral energy distributions (SEDs). Finally, the conclusions of our study are presented in Section 4.

\section{OBSERVATIONS AND DATA ANALYSIS}

\subsection{Collection of supernova data from the Spitzer Heritage Archive}

Using the list of SNe on the website of Central Bureau for Astronomical Telegrams $(\mathrm{CBAT})^{1}$, and the website of All-Sky Automated Survey for Supernovae (ASAS-SN Shappee et al. 2014; Holoien et al. 2017a,b,c) $)^{2}$, we selected all SNe that were discovered before 2015 and have been spectroscopically classified. We also selected additional nearby $(z \lesssim 0.05)$ SNe listed in the Open Supernova Catalog ${ }^{3}$ (Guillochon et al. 2017). This search returned $\sim 4500$ objects, all of which have had their positions searched in the Spitzer Heritage Archive (SHA) ${ }^{4}$ (using a 100" environment for the queries). We found $1142 \mathrm{SN}$ sites that have been observed post-explosion with Spitzer. For these SNe, we downloaded the available IRAC data for further analysis whether or not the data had been previously published. We note that although MIPS (Multiband Imaging Photometer) and IRS (Infrared Spectrograph) data can also contribute to the understanding of the mid-IR behavior of SNe (see e.g. Kotak et al. 2006, 2009; Gerardy et al. 2007; Fabbri et al. 2011; Szalai et al. 2011; Szalai \& Vinkó 2013), only

\footnotetext{
${ }^{1}$ http://www.cbat.eps.harvard.edu/lists/Supernovae.html

${ }^{2}$ http://www.astronomy.ohio-state.edu/ assassin

${ }^{3}$ https://sne.space

${ }^{4}$ http://sha.ipac.caltech.edu
} 
a few objects observed with these instruments exist so we focus only on IRAC data in this article.

\subsection{Object identification and photometry on Spitzer/IRAC images}

We collected and analyzed all available IRAC post-basic calibrated data (PBCD). The scale of these images is $0.6^{\prime \prime} /$ pixel. Identifying a point source at the position of an SN explosion can be difficult at the large distances to some of these galaxies, where compact H II regions or the host clusters of SNe may also appear as point-like sources on Spitzer/IRAC images. Furthermore, the target can be faint or on top of a complex background. We therefore performed image subtraction with HOTPANTS ${ }^{5}$ whenever a template exists (Fig. 1). This procedure achieved a good match between the background levels of target and template frames, resulting in net background levels close to zero in the subtracted images. However, not all targets have templates. In these cases, local background was estimated by measuring actual flux fluctuations via placing apertures covering the region of the SN site. In all cases (including either image-subtracted or non-subtracted images), we defined the source as a positive detection if i) the source showed epoch-to-epoch flux changes, and ii) its flux was above the local background by at least $5 \mu \mathrm{Jy}$ and $15 \mu \mathrm{Jy}$ at 3.6 and $4.5 \mu \mathrm{m}$, respectively (according to point-source sensitivities in Table 2.10 of the IRAC Instrument Handbook version 2).

Moreover, in some cases, only a single-epoch set of Spitzer observations is available, thus, epoch-to-epoch flux changes cannot be used as indicators of the presence of SNe. In these cases, as a first step, we used archival pre-explosion 2MASS JHK ages in order to exclude the potential false-positive detections (compact H II regions etc.). For the precise astrometric comparison, we collected the absolute coordinates of the concerned SNe from the Open Supernova Catalog and derived their (x,y) coordinates in the Spitzer/IRAC images (note that the uncertainties of the absolute SN coordinates have not been reported in the most cases). Spitzer/IRAC post-BCD images have a pointing to $2 \mathrm{MASS}$ with an accuracy of $0.15^{\prime \prime}$ (see IRAC Instrument $\mathrm{Handbook}^{6}$ ); an additional limit is the $0.6^{\prime \prime} /$ pixel resolution of Spitzer/IRAC PBCD images. The basic astrometric criterion of a potential positive detection was an agreement between the absolute SN coordinates and the position of the photometric center of the mid-IR point source within two IRAC pixels $\left(1.2^{\prime \prime}\right)$. In the second step, we carried out aperture photometry on the pre-explosion 2MASS JHKs images (using the same aperture and annulus/dannulus parameters as during the Spitzer photometry). Since, in most cases, there are no detectable point sources on the 2MASS images at the positions of the $\mathrm{SNe}$, it was not possible to estimate reliable photometric errors based on photon statistics; instead, we have used a \pm 0.4 mag value as a general photometric error, based on the upper limit of 2MASS photometric uncertainties reported

\footnotetext{
${ }^{5}$ http://www.astro.washington.edu/users/becker/hotpants.html

${ }^{6}$ https://irsa.ipac.caltech.edu/data/SPITZER/docs/irac/iracinstrumenthandbook/
} 
in Skrutskie et al. (2006). In order to reveal the presence of any possibly real mid-IR excess at post-explosion Spitzer/IRAC images, we have fitted simple blackbodies to the SEDs consist of the upper limits of pre-explosion 2MASS photometry (assuming a general uncertainty of 0.4 mag mentioned above). The photometric criterion of a positive detection was to find Spitzer/IRAC fluxes being above the fitted SED with a 3- $\sigma$ photometric error in at least one IRAC channel. Conclusively, we labeled in total 7 SNe with single-epoch Spitzer data as positive detections; we note that all of these SNe are expected to show strong mid-IR radiation at the given epoch (strongly interacting SNe IIn, or early-caught SNe of other types). We present all the pairs of images (Spitzer/IRAC + pre-explosion 2MASS $\mathrm{K}_{s}$ ) and SED fittings lead us to select the single-epoch positive detections, together with an example for negative detections, in Appendix B.

We performed a photometric analysis for all positive detections at every epoch. For isolated sources, we implemented aperture photometry on the PBCD frames using the phot task of $\mathrm{IRAF}^{7}$ as a first step. We generally used an aperture radius of $2^{\prime \prime}$ and a background annulus from $2^{\prime \prime}$ to $6^{\prime \prime}$ (2-2-6 configuration), applied aperture corrections of $1.213,1.234,1.379$, and 1.584 for the four IRAC channels $(3.6,4.5$, 5.8, and $8.0 \mu \mathrm{m}$, respectively) as given in IRAC Data Handbook, but sometimes used the 3-3-7 configuration (aperture corrections: 1.124, 1.127, 1.143, and 1.234, respectively) or the 5-12-20 configuration (aperture corrections: 1.049, 1.050, 1.058, and 1.068, respectively). For targets with templates, we compared the results before and after template subtraction to test for consistency. We generally found good agreement between the two methods $(\lesssim 10 \%$ difference in fluxes, which is within the approximated uncertainty of Spitzer/IRAC photometry). In the few cases where the difference between the two methods was more than $10 \%$, we preferred the results of image subtraction photometry.

For sources on top of complex backgrounds without a corresponding template, we implemented the photometric method described by Fox et al. (2011) (called hereafter as "Fox+11 method"). This method applies a set of single apertures with a fixed radius to estimate both the $\mathrm{SN}$ and average background flux. This technique allows us to visually identify only local background associated with the $\mathrm{SN}$, as opposed to the annuli regarding the aperture configurations mentioned above.

We compared our results to any previously published Spitzer/IRAC SN-photometry. In general, we found good agreements with the published values $(\lesssim 10 \%$ difference in fluxes). In a few cases, the flux differences are larger, but each of these cases consist of either a very faint target and/or complex sky background.

The target details and resulting mid-IR photometry of all SNe with previously unpublished Spitzer photometry are listed in Tables A1 and A2, respectively. We clearly highlight SNe identified on a single-epoch set of Spitzer images, as well as

${ }^{7}$ IRAF is distributed by the National Optical Astronomy Observatories, which are operated by the Association of Universities for Research in Astronomy, Inc., under cooperative agreement with the National Science Foundation. 

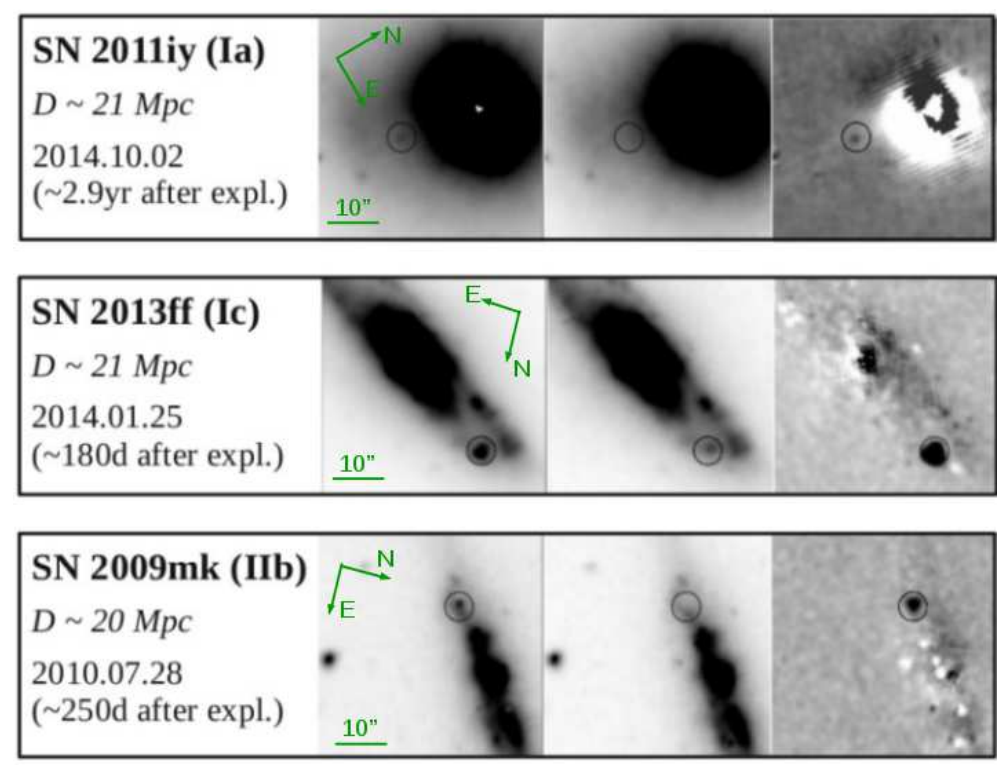

Figure 1. HOTPANTS template subtraction of our Spitzer data. For each SN, the three panels show (left) the most recent Spitzer/IRAC $4.5 \mu \mathrm{m}$ image, (center) template, and (right) differenced image.

all the other SNe where image subtraction can not be applied; in all these cases, measured fluxes are strictly handled as upper limits. Flux uncertainties in Table A2 generally based on photon statistics provided by phot, but, where photometry was carried out on subtracted images, increment of the noise level by $\sqrt{2}$ is also taken into account.

\section{RESULTS}

\subsection{Demographics}

The total number of the observed SN positions is over 1100. The majority of SNe are nearby $(\mathrm{z}<0.05)$. We detect $119 \mathrm{SNe}$, including 45 objects that have no previously published Spitzer photometry. Only $\sim 12 \%$ of the SN sites were observed pre-explosion. We also highlight three specific targets (SNe 2012aw, 2012fh, and 2013ee), which have been noted by Tinyanont et al. (2016) as positive Spitzer detections, but without any corresponding photometry. We summarize the statistics of our SN sample in Table 1 and Figs. 2 and 3.

About $40 \%$ of the objects (mostly Type Ia) are located in distant, anonymous galaxies, but these observations did not yield any SN detections. There are also $\sim 60$ SNe that are also located in complex regions of the galaxy, typically very close to the galaxy nuclei. In these cases, even template subtraction is not effective due to the asymmetric profile of the IRAC point-spread function (PSF). We do not include any of these SNe in this analysis.

Following the methods presented by Tinyanont et al. (2016), we present the detection rates separated in three time bins after discovery: less than one year, one to three 
years, and more than three years. If a SN is observed with at least one detection in a bin, it is considered detected, even though it might fade away later in the same bin. 
Table 1. Statistics of the Spitzer/IRAC data regarding the sample of studied SNe.

\begin{tabular}{|c|c|c|c|c|c|c|c|c|c|c|c|c|c|c|c|c|}
\hline \multirow{4}{*}{$\begin{array}{l}\text { Total number of } \\
\text { observed SN sites }\end{array}$} & \multicolumn{16}{|c|}{ Total number of observed SN sites: $1142 / 693^{\dagger}$} \\
\hline & \multicolumn{4}{|c|}{ Thermonuclear SNe } & \multicolumn{7}{|c|}{ Stripped-envelope CC SNe } & \multicolumn{5}{|c|}{ Type II SNe } \\
\hline & Ia & Ia-pec & $\operatorname{Iax}$ & Ia-CSM & $\mathrm{Ib}$ & Ib-pec & Ibn & $\mathrm{Ib} / \mathrm{c}$ & Ic & Ic-pec & IIb & II-P & II-P pec. & IIn & II-L & Unclass. SN II \\
\hline & $723 / 294^{\dagger}$ & $25 / 23^{\dagger}$ & 8 & 5 & $59 / 53^{\dagger}$ & 1 & 2 & 1 & $73 / 63^{\dagger}$ & $5 / 4^{\dagger}$ & 25 & 36 & 2 & 101 & 4 & 72 \\
\hline \multirow{4}{*}{$\begin{array}{c}\text { SN sites with } \\
\text { multiple observations }\end{array}$} & \multicolumn{16}{|c|}{ SN sites with multiple observations: $553 / \mathbf{3 3 4}^{\dagger}$} \\
\hline & \multicolumn{4}{|c|}{ Thermonuclear SNe } & \multicolumn{7}{|c|}{ Stripped-envelope CC SNe } & \multicolumn{5}{|c|}{ Type II SNe } \\
\hline & Ia & Ia-pec & Iax & Ia-CSM & $\mathrm{Ib}$ & Ib-pec & Ibn & $\mathrm{Ib} / \mathrm{c}$ & Ic & Ic-pec & IIb & II-P & II-P pec. & IIn & II-L & Unclass. SN II \\
\hline & $325 / 112^{\dagger}$ & 9 & 4 & 5 & $27 / 25^{\dagger}$ & 1 & 1 & - & $35 / 33^{\dagger}$ & 3 & 14 & 32 & 2 & 38 & 4 & 53 \\
\hline \multirow{4}{*}{$\begin{array}{c}\text { SN sites with } \\
\text { pre-explosion images }\end{array}$} & \multicolumn{16}{|c|}{ SN sites with pre-explosion images: $111 / 87^{\dagger}$} \\
\hline & \multicolumn{4}{|c|}{ Thermonuclear SNe } & \multicolumn{7}{|c|}{ Stripped-envelope CC SNe } & \multicolumn{5}{|c|}{ Type II SNe } \\
\hline & Ia & Ia-pec & $\operatorname{Iax}$ & Ia-CSM & $\mathrm{Ib}$ & Ib-pec & Ibn & $\mathrm{Ib} / \mathrm{c}$ & Ic & Ic-pec & $\mathrm{IIb}$ & II-P & II-P pec. & IIn & II-L & Unclass. SN II \\
\hline & $43 / 20^{\dagger}$ & 3 & 2 & - & 10 & - & 1 & - & $9 / 8^{\dagger}$ & - & 4 & 10 & 2 & 9 & 1 & 17 \\
\hline \multirow{4}{*}{$\begin{array}{c}\text { Total number of } \\
\text { positive detections }\end{array}$} & \multicolumn{16}{|c|}{ Total number of positive detections: 119} \\
\hline & \multicolumn{4}{|c|}{ Thermonuclear SNe } & \multicolumn{7}{|c|}{ Stripped-envelope CC SNe } & \multicolumn{5}{|c|}{ Type II SNe } \\
\hline & Ia & Ia-pec & $\operatorname{Iax}$ & Ia-CSM & $\mathrm{Ib}$ & Ib-pec & Ibn & $\mathrm{Ib} / \mathrm{c}$ & Ic & Ic-pec & $\mathrm{IIb}$ & II-P & II-P pec. & IIn & II-L & Unclass. SN II \\
\hline & 24 & 1 & 2 & 5 & 5 & - & 1 & 1 & 7 & 1 & 7 & 22 & 1 & 25 & 2 & 15 \\
\hline \multirow{4}{*}{$\begin{array}{c}\text { Unpublished } \\
\text { positive detections }\end{array}$} & \multicolumn{16}{|c|}{ Unpublished positive detections: 45} \\
\hline & \multicolumn{4}{|c|}{ Thermonuclear SNe } & \multicolumn{7}{|c|}{ Stripped-envelope CC SNe } & \multicolumn{5}{|c|}{ Type II SNe } \\
\hline & Ia & Ia-pec & $\operatorname{Iax}$ & Ia-CSM & $\mathrm{Ib}$ & Ib-pec & Ibn & $\mathrm{Ib} / \mathrm{c}$ & Ic & Ic-pec & $\mathrm{IIb}$ & II-P & II-P pec. & IIn & II-L & Unclass. SN II \\
\hline & 13 & 1 & 1 & 2 & 3 & - & - & 1 & 2 & - & 4 & 4 & - & 6 & 1 & 7 \\
\hline
\end{tabular}

Note ${ }^{\dagger}$ Total number of objects / Number of objects excluding SNe in distant, anonymous galaxies 

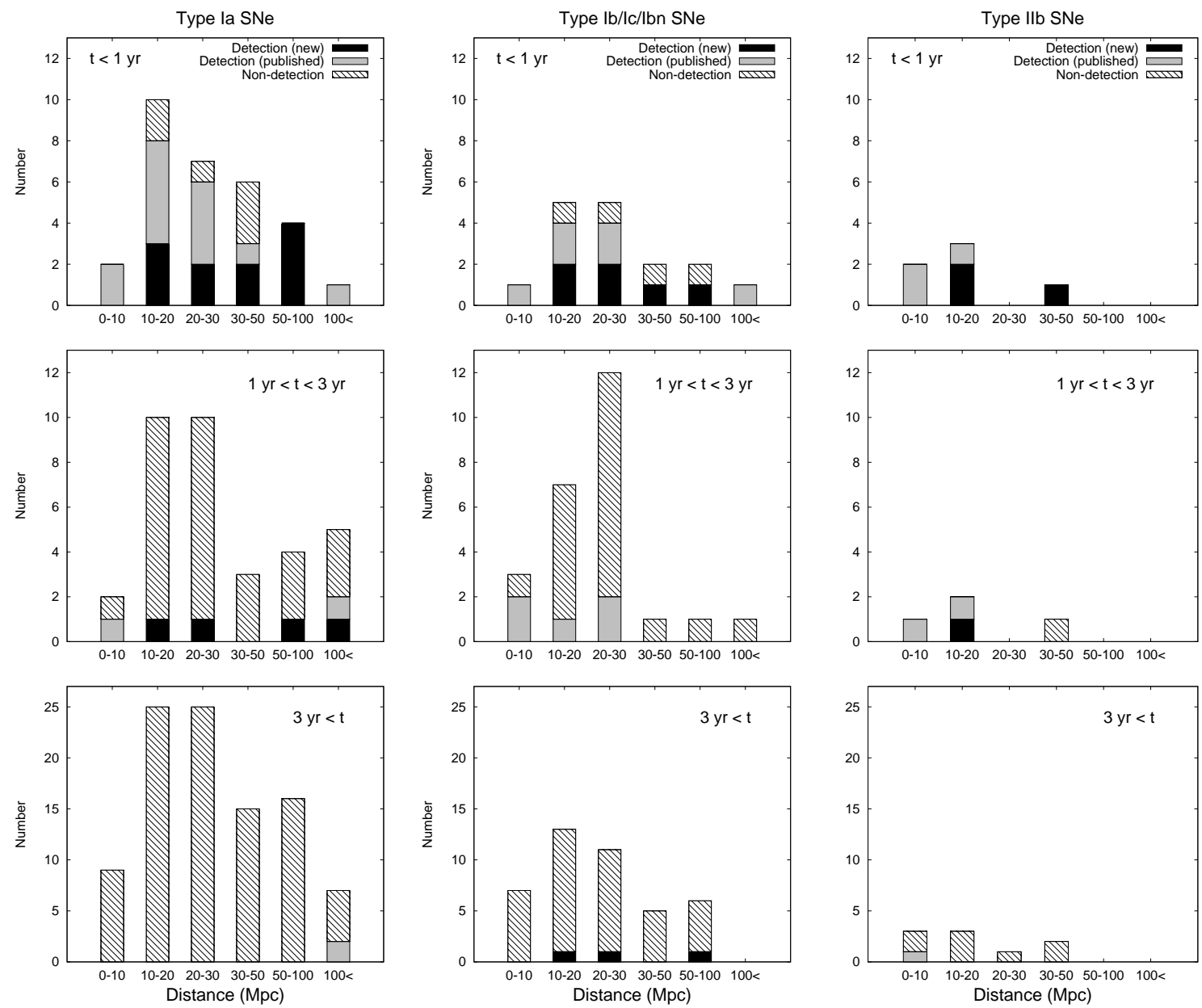

Figure 2. The statistics of detected Type Ia and stripped-envelope CC SNe in our Spitzer/IRAC sample. The statistics are divided by type and epoch. The number of detections is plotted as a function of distance in each case. We do not include SNe located in distant $(z \gtrsim 0.05)$, anonymous galaxies and/or too close to the center of their hosts. We also exclude most SNe with only a single-epoch Spitzer/IRAC observations.

\subsection{Mid-IR evolution: trends and outliers}

Fig. 4 plots the mid-IR photometry of all SNe with positive Spitzer detections. Table A3 lists all corresponding Vega magnitudes, distances, and $E(B-V)$ values. For plotting purposes, Fig. 4 excludes some objects with decade-long mid-IR datasets - e.g. Type II-pec SN 1987A (Dwek et al. 2010), or Type II-L SN 1979C (Tinyanont et al. 2016) -, but these SNe are included in our statistical analysis. Figs. 5-9 highlight the SN subclasses so that individual SNe can be identified and photometric details can be ascertained. Tables A1 and A3 contain all the sources of previously published Spitzer data we used for constructing Figs. 2-9 and for the analysis we present below.

\subsubsection{Thermonuclear $S N e$}

This work more than doubles the number of SNe Ia with positive mid-IR detection (33 vs. 15). Fig. 5 shows that most Type Ia SNe have a relatively well-defined evolution compared to the other SN subclasses, consistent with previous results 

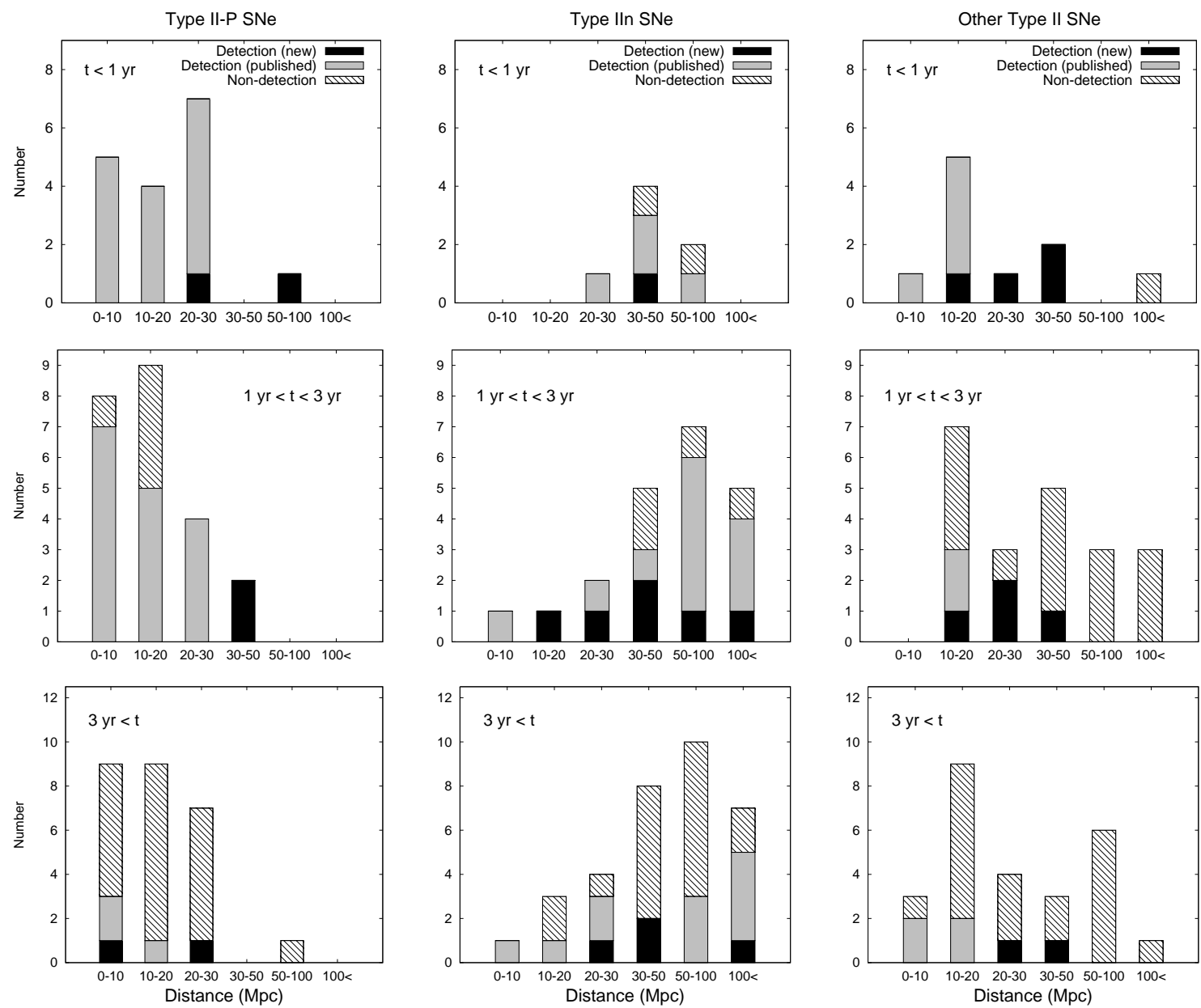

Figure 3. Same as Figure 2, except in this case for SNe II-P, Type IIn, and other (unclassified) Type II SNe.

(Tinyanont et al. 2016; Johansson et al. 2017). The handful of Type Ia-CSM SNe, however, are extremely bright at mid-IR wavelengths. This sample is small, so it is difficult to draw any definitive conclusions about the overall trend. For example, PTF11kx ( $D \sim 200 \mathrm{Mpc})$ is still detectable at $\sim 1800$ days, while SN 2002ic ( $D \sim 280$ Mpc) faded at a similar age.

We do not find any previously unpublished SNe Ia with mid-IR fluxes comparable to those of the known SNe Ia-CSM. This result suggests that a dense CSM is rare in the environments of thermonuclear SNe (which may also hint that SNe Ia-CSM arise from different progenitor systems than the majority of SNe Ia), or, that CSM shells may be far away from the explosion sites. Based on the existing (rough) estimations, SN Ia-CSM objects may contribute between $1 \%$ and $5 \%$ of all SNe Ia (see e.g. Meng \& Podsiadlowski 2018), which seems to be supported by our results; however, future systematic surveys are necessary for the thorough study of this problem.

We do find some other SNe Ia that deviate from the expected mid-IR evolution. SNe 2010B and 2010gp, observed at early times, are noticeably brighter. Note, however, that SN 2010B has a complex background that may be contributing additional mid-IR 


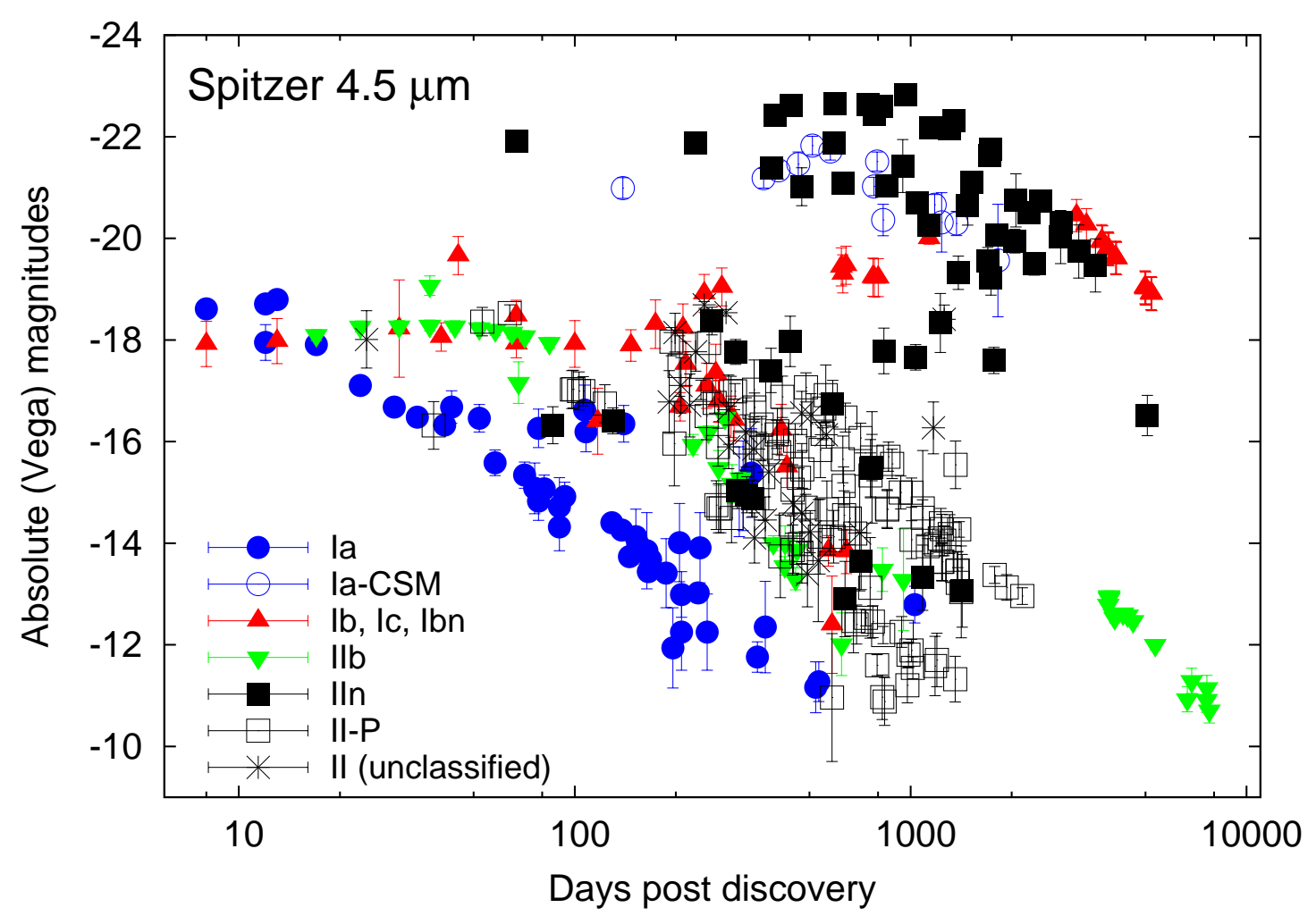

Figure 4. $4.5 \mu \mathrm{m}$ absolute Vega magnitudes of all $\mathrm{SNe}$ identified as point sources in Spitzer/IRAC images. Values and sources of data are shown in Table A3.

flux. On the other hand, SN 2010gp has a template for subtraction, so these results are quite robust. SN 2011iy, which is relatively nearby ( $d \sim 20 \mathrm{Mpc}$ ), also appears as a point source at $4.5 \mu \mathrm{m}$ after image subtraction at $\sim 1030$ days after explosion (see Fig. 1). This SN, however, is not detectable at $3.6 \mu \mathrm{m}$.

SN 2014dt, classified as a Type Iax SN, should be also highlighted here: this object shows a clear and even growing mid-IR excess $\sim 1$ yr after explosion, which has been explained with the presence of newly-formed dust, pre-existing dust, or possibly a bound remnant (Fox et al. 2016; Foley et al. 2016). The only other SN Iax we identified as a mid-IR source on Spitzer images is SN 2005P. While SN 2005P seems to have a slight $8.0 \mu$ m detection at $\sim 180$ days, there is no comparable data with SN $2014 \mathrm{dt}$. By $\sim 1$ year post-explosion, SN 2005P has faded below the detection threshold.

\subsubsection{Stripped-envelope CC SNe}

Fig. 6 plots the mid-IR absolute magnitudes of SE CCSNe. The stripped-envelope designation encompasses various subclasses, including SNe IIb, Ib, and Ic, so their mid-IR evolution exhibits a bit of heterogeneity, particularly at later times.

The mid-IR evolution of "normal" Type Ib/Ic SNe seem to be fastest amongst SE CCSNe. SN 2014C presents itself as a special case in which the explosion transforms from a "normal" Type Ib into a strongly-interacting, Type In-like SN (Milisavljevic et al. 2015; Margutti et al. 2017). SN 2014C is located within 


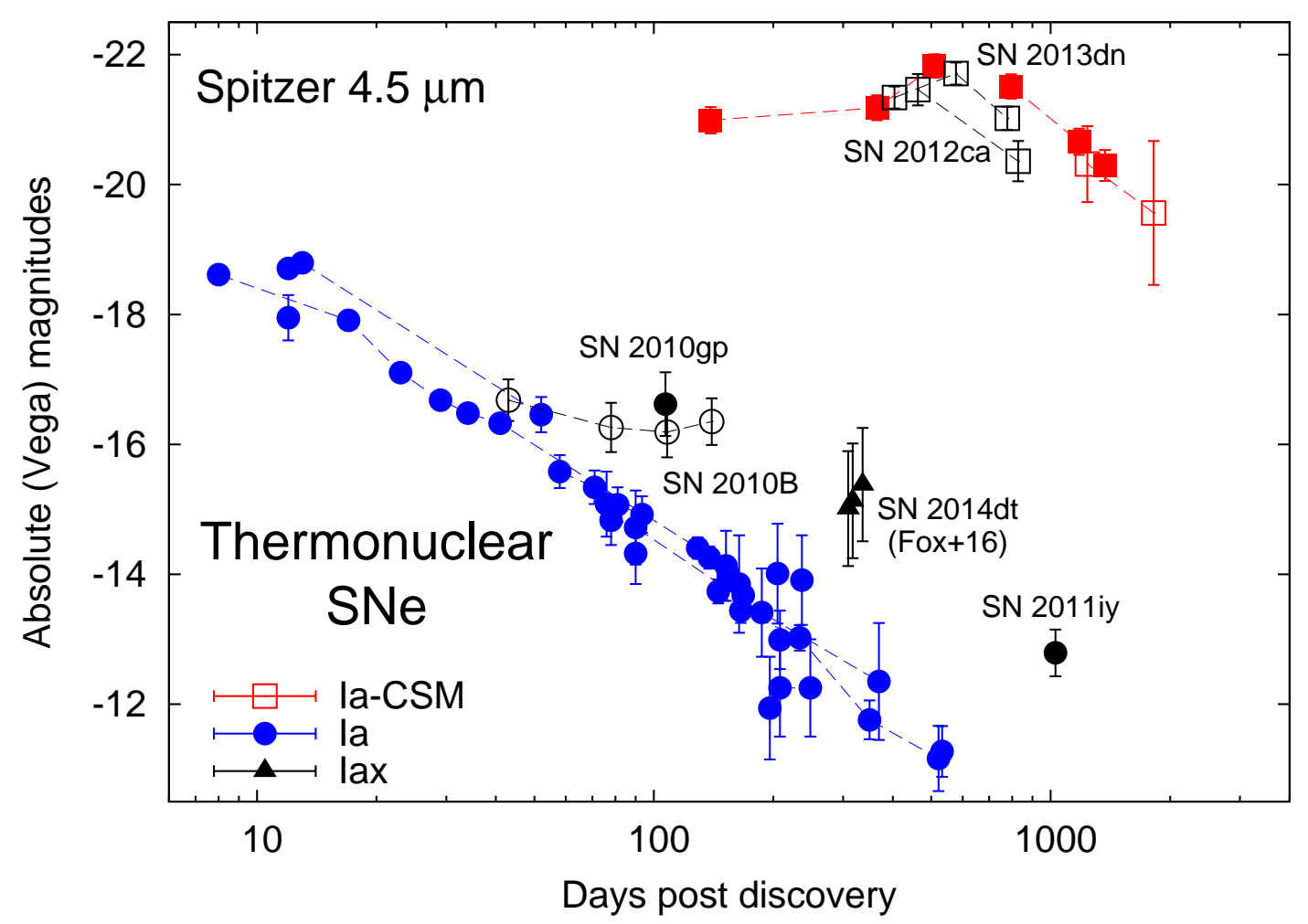

Figure 5. Mid-IR evolution of thermonuclear SNe; highlighted objects are marked with black symbols, while filled and empty symbols denote SNe whose absolute magnitudes were determined with or without template subtraction, respectively. Values and sources of data are shown in Table A3.

NGC 7331, which has been followed extensively as part of the SPIRITS program. Tinyanont et al. (2016) show a roughly constant IR-luminosity in the first $~ 800$ days and a unique re-brightening at $\sim 250$ days as the CSM interaction begins.

Another interesting object is SN 2001em, a strongly-interacting Type Ib/c object, which generated strong X-ray, radio and optical emission for $\sim 3$ years postexplosion (see Stockdale et al. 2004; Pooley \& Levin 2004; Soderberg et al. 2004; Chugai \& Chevalier 2006). Unlike SN 2014C, however, the transformational process was not observed by Spitzer, making a direct comparison impossible. SN 2001em was observed by Spitzer only once. Fig. 6 shows SN 2001em is even brighter than SN 2014C, although background contributions have not been removed. We present a more detailed analysis of SN 2001em in Section 3.3.

Finally, it is worth mentioning the one observation of SN $2011 \mathrm{ft}$, a distant $(d \sim 100$ Mpc) Type Ib SN that is as bright as SN 2014C at $~ 250$ days after explosion. With only a single $3.6 \mu \mathrm{m}$, more observations are planned.

Observations exist for only two SNe Ibn: SN 2006jc (4 epochs, but only one from the first year) and PS1-12sk (1 epoch). These two events are bright in mid-IR during the early-time CSM interaction, but the brightness declines quickly. Following the interpretation of Mattila et al. (2008), the early mid-IR radiation may arise from 


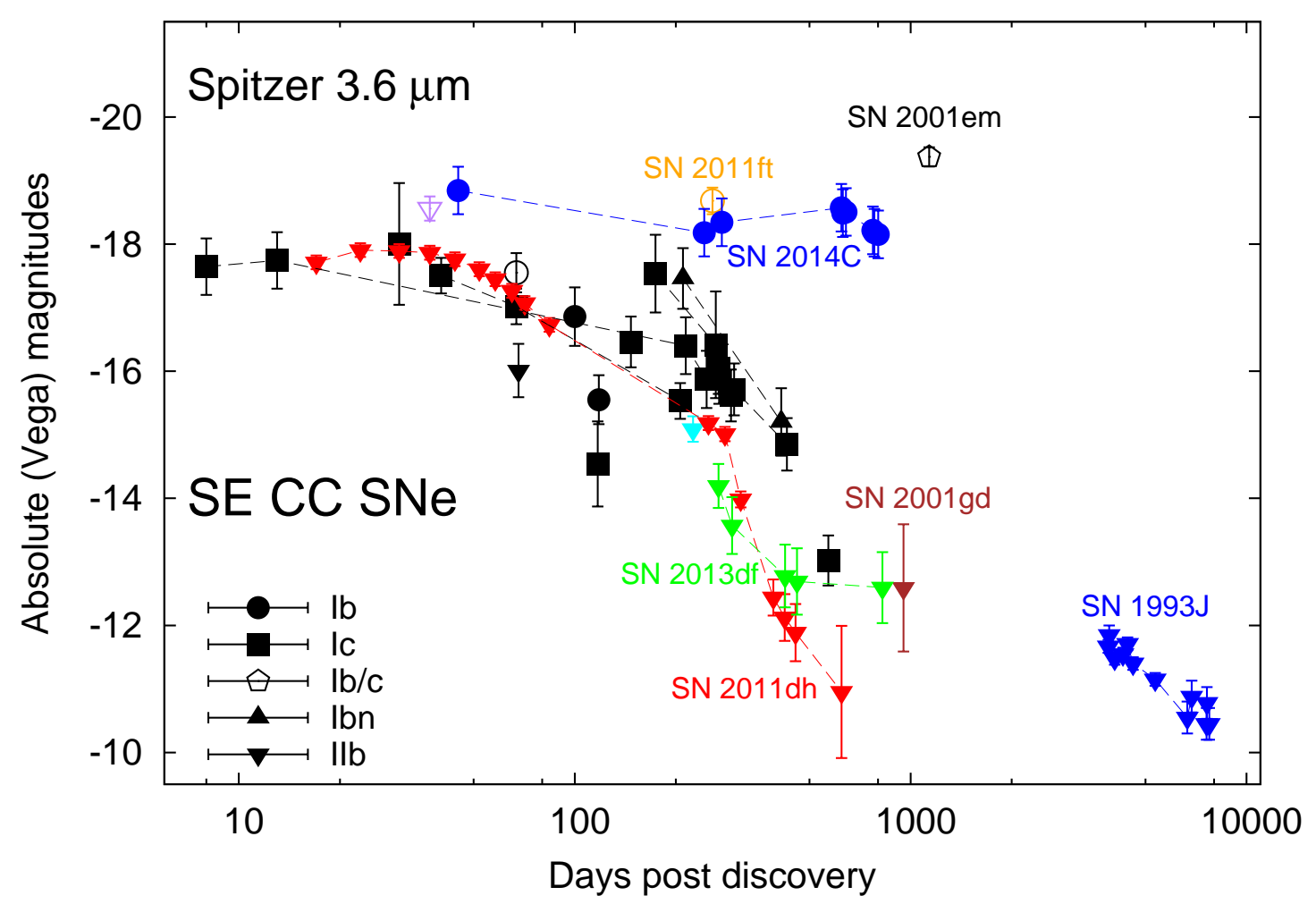

Figure 6. Mid-IR evolution of stripped-envelope core-collapse SNe; highlighted objects are marked with labels, while filled and empty symbols denote SNe whose absolute magnitudes were determined with or without image subtraction, respectively. Values and sources of data are shown in Table A3.

newly formed dust in the CDS, while the source of the later-time mid-IR flux is probably an IR echo from pre-existing dust in the CSM.

Among SNe IIb, the moderately interacting SN 2013df (Kamble et al. 2016; Maeda et al. 2015b; Szalai et al. 2016) produces a slowly-declining mid-IR light curve between $\sim 270-820$ days (Tinyanont et al. 2016; Szalai et al. 2016). SN 2001gd shows a similar brightness at $\sim 950$ days. SN 2011dh, one of the best-sampled SN in midIR, has been also detectable up to almost two years after explosion. The Type IIb SN 1993J ( $D \sim 3.7 \mathrm{Mpc}$ ) is detected at $>24$ years post-explosion in mid-IR (Tinyanont et al. 2016), while the Type IIb SN 2008ax ( $D \sim 7.8 \mathrm{Mpc}$ ) is not detected at even $\sim 4$ years after explosion.

The differences between SNe IIb seem to correlate with the assumed sizes of the progenitors of SE CCSNe. SNe 1993J, 2001gd and 2013df, which are detected by Spitzer at later epochs, have been classified as Type eIIb objects (Chevalier \& Soderberg 2010; Szalai et al. 2016), which denotes that these explosions originate from extended progenitors (yellow or red giants). SN 2008ax is known as a representative of Type cIIb objects, which are defined to have more compact progenitors, similar to those of $\mathrm{SNe} \mathrm{Ib} / \mathrm{c}$. SN 2011dh seems to be an intermediate case in both its progenitor radius ( $R \sim$ a few tens of $R_{\odot}$ ) and mid-IR evolution. 


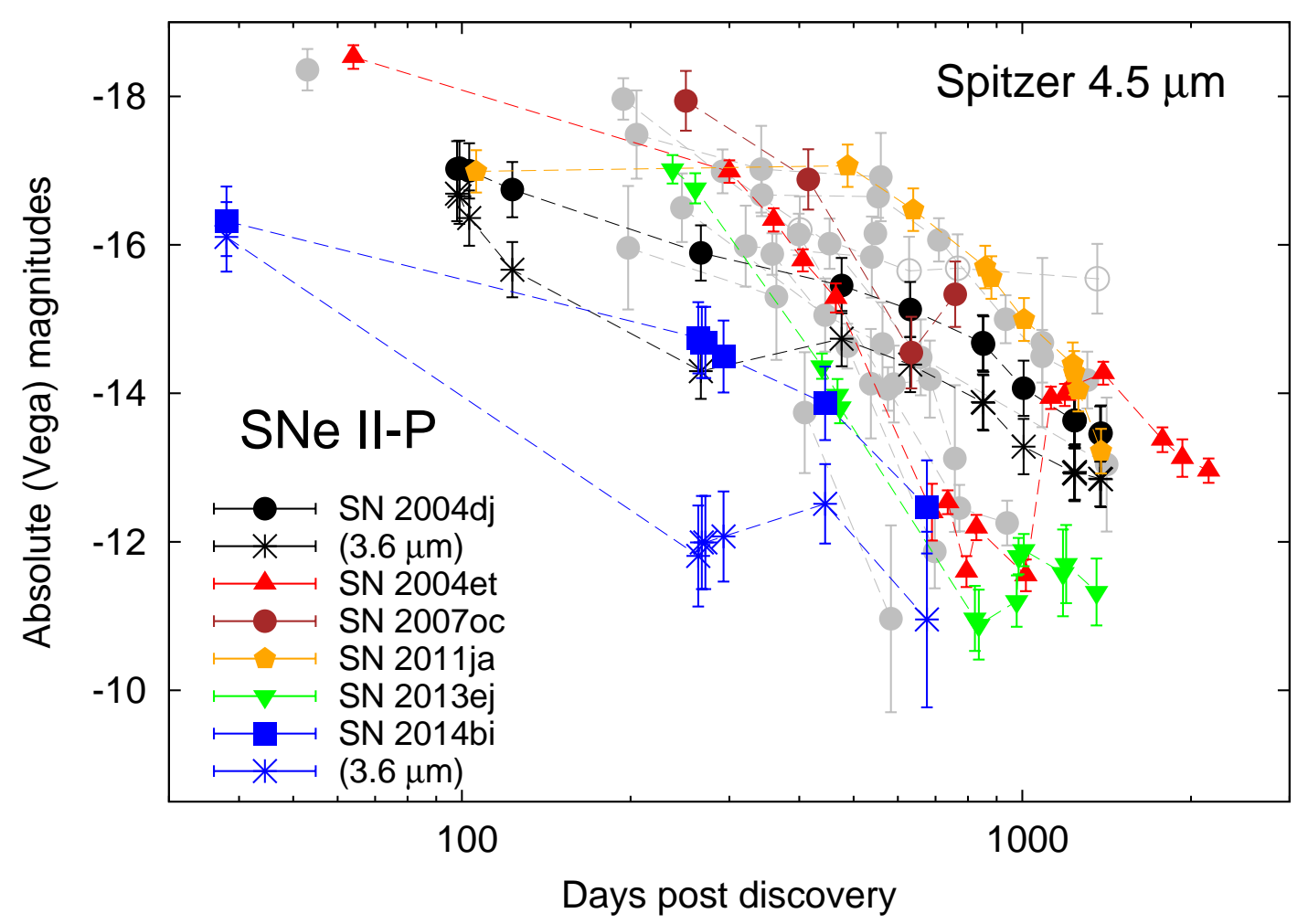

Figure 7. $4.5 \mu \mathrm{m}$ absolute magnitudes of Type II-P explosions. Colored symbols denote objects where mid-IR rebrightening occurred. Filled and empty symbols denote SNe whose absolute magnitudes were determined with or without image subtraction, respectively. In two cases (SNe 2004dj and 2014bi), re-brightening can be only observed at $3.6 \mu \mathrm{m}$ (see details in text), which curves are also shown (marked with asterisks). All data are shown in Table A3.

\subsubsection{Type II-P SNe}

Fig. 7 plots the mid-IR absolute magnitudes of Type IIP SNe, which show a relatively homogeneous mid-IR evolution. Theoretical models suggest that the ejecta of most of Type II-P SNe may form dust between 300-600 days due to the slow expansion velocities and high densities. Only a few SNe show evidence for a rebrightening in the mid-IR between 300-600 days: SNe 2004dj (Szalai et al. 2011; Meikle et al. 2011), 2011ja (Andrews et al. 2016; Tinyanont et al. 2016), and 2014bi (Tinyanont et al. 2016). This unexpectedly low rate may be influenced by the poor sampling of the other observed Type IIP SNe. Furthermore, while both SNe 2004dj and 2014bi show the rebrightening effect at $3.6 \mu \mathrm{m}$ (the first object even at 5.8 and $8.0 \mu \mathrm{m}$, see Szalai et al. 2011), it is not detectable at $4.5 \mu \mathrm{m}$ (there is a linear flux decline instead). Szalai et al. (2011) suggest that additional flux at $4.5 \mu \mathrm{m}$ arises from the 1-0 vibrational band of $\mathrm{CO}$ at $4.65 \mu \mathrm{m}$ (see Kotak et al. 2005) during the declining phase, but disappears at 500d (Szalai et al. 2011; Szalai \& Vinkó 2013), thereby making $4.5 \mu \mathrm{m}$ light-curves difficult to interpret for SNe II-P. 
Two other Type IIP SNe, 2004et (Kotak et al. 2009; Fabbri et al. 2011) and 2007oc (Szalai \& Vinkó 2013), as well as Type IIP/IIL SN 2013ej (Tinyanont et al. 2016; Mauerhan et al. 2017) also show mid-IR rebrightening, but it occurred between $\sim 700$ 1000 days. This rebrightening is detected at both 3.6 and $4.5 \mu \mathrm{m}$ (at least in the cases of SNe 2004et and 2007oc; SN 2013ej becomes undetectable at $3.6 \mu \mathrm{m}$ after $\sim 800$ days). The above papers suggest this rebrightening is due to new dust forming in the CDS behind the reverse shock and not within the ejecta.

\subsubsection{Type IIn SNe}

Fig. 8 plots the mid-IR absolute magnitudes of SNe IIn. For most SNe IIn, Fox et al. (2011, 2013) show that the mid-IR radiation arises from pre-existing dust, which is radiatively heated by optical emission generated by ongoing interaction between the forward shock and CSM. While many SNe IIn show early evidence for CSM interaction (e.g., strong emission in $\mathrm{H} \alpha$ / X-ray / radio), only a hand-full of Spitzer observations exist in the first few months post-explosion. SNe 2009ip (Fraser et al. 2015, and this work) and 2011A were faint mid-IR sources in the first months, but both of these objects are considered low-luminosity Type IIn events/impostors (see the analyses of e.g. Pastorello et al. 2013, Fraser et al. 2013, Mauerhan et al. 2013, and Margutti et al. 2014, and de Jaeger et al. 2015). By contrast, SN 2010jl was extremely bright in mid-IR during the first year (Andrews et al. 2011a; Fox et al. 2013; Fransson et al. 2014; Williams \& Fox 2015). The origin of the mid-IR excess has been debated, but is likely a combination of both newly formed and pre-existing dust (Gall et al. 2014; Fransson et al. 2014).

The mid-IR evolution of SNe IIn is heterogeneous. While many SNe IIn remain bright for year post-explosion, the decline rates are not always the same. Furthermore, many SNe IIn are not even detected (see Fig. 3, as well as Fox et al. 2011). These differences likely correspond to the extent of pre-SN mass-loss, but may also suggest different geometries, shock velocities, and progenitors.

Fig. 4 shows that Type II-P and Type IIn SNe have quite distinct late-time midIR evolution. This dichotomy serves as a useful classification method for several unclassified targets in our sample (see Table 1). Most of these sources are likely Type IIP, except SNe 2005kd, 2008fq, and 2011dq, which may SNe IIn, as it is shown in Fig. 9.

\subsection{SED fittings: limitations, methods, consequences}

Mid-IR SEDs of SNe span the peak of the thermal emission from warm dust and can place useful constraints on the dust properties (see e.g. Kotak et al. 2009; Szalai et al. 2011; Szalai \& Vinkó 2013). In most of our sample, these fits are limited to only two photometry points. Further challenges exist. During the first several months after explosion, a hot component arising from an optically thick gas in the innermost part of the ejecta may affect the continuum emission at these wavelengths. Moreover, the 


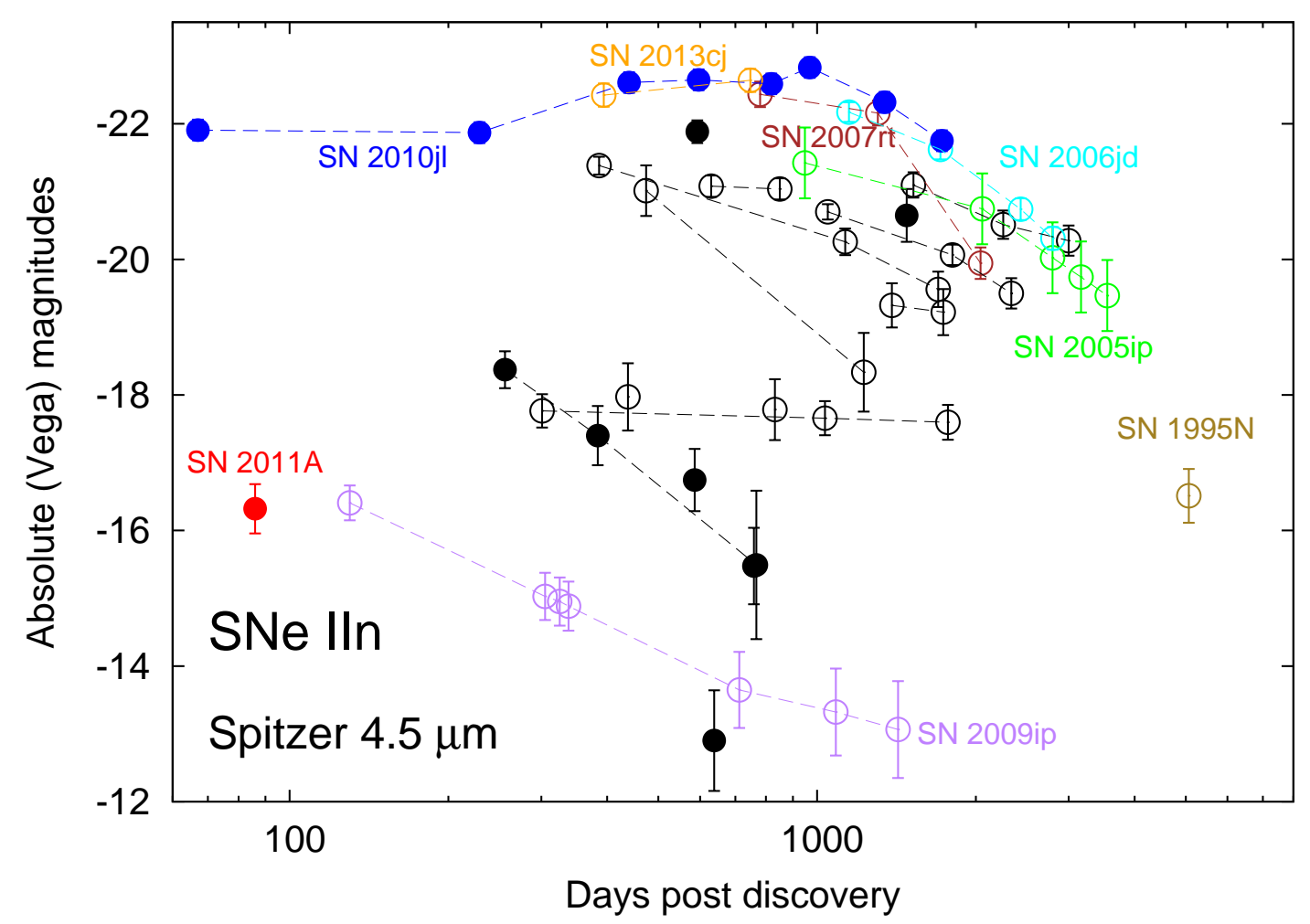

Figure 8. Mid-IR evolution of the studied Type IIn explosions. Highlighted objects are marked with colored symbols (see details in text). Filled and empty symbols denote SNe whose absolute magnitudes were determined with or without image subtraction, respectively. In the case of SN 2009ip, epochs are defined relative to the large outburst occurred in 2012. All the data are shown in Table A3.

line emission by $\mathrm{CO}$ at $4.65 \mu \mathrm{m}$ (described in Section 3.2.3) may also contribute a significant flux at $4.5 \mu \mathrm{m}$ (although this effect has only been observed in some Type II-P SNe before $\sim 500$ days after explosion). Most of our sample with previously unpublished Spitzer data lack the multi-wavelength data that can improve these fits. We also note that while the Galactic extinction (typically at a level below $E(B-$ $V)=0.1$, see Table A1) is practically negligible at mid-IR wavelengths, the host galaxy extinction can be more important for both thermonuclear (see e.g. Phillips et al. 2013) and core-collapse SNe (see e.g. Jencson et al. 2018, and references therein). Unfortunately, regarding most of the studied SNe, we have no information about the host extinction. As a simple estimation (based on the results of Xue et al. 2016), an extreme value of $E(B-V)_{\text {total }}=1.0$ mag can attenuate the measured flux by $\sim 20 \%$ at 3.6 or $4.5 \mu \mathrm{m}$.

We illustrate our fitting process using data from the SN IIn 2011A and SN II-P 2014cx. Both of these objects were observed by Spitzer within 3 months after explosion (at +86 and +53 days, respectively). In the case of SN 2011A, contemporaneous $g^{\prime} r^{\prime} i{ }^{\prime} z$ ' data can be found in de Jaeger et al. (2015), and in the case of SN 2014cx, BVRI and g'r' $i$ ' data obtained at the epoch of Spitzer observations can be found in Huang et al. (2016). The mid-IR fluxes were transformed to $F_{\lambda}$ values and dered- 


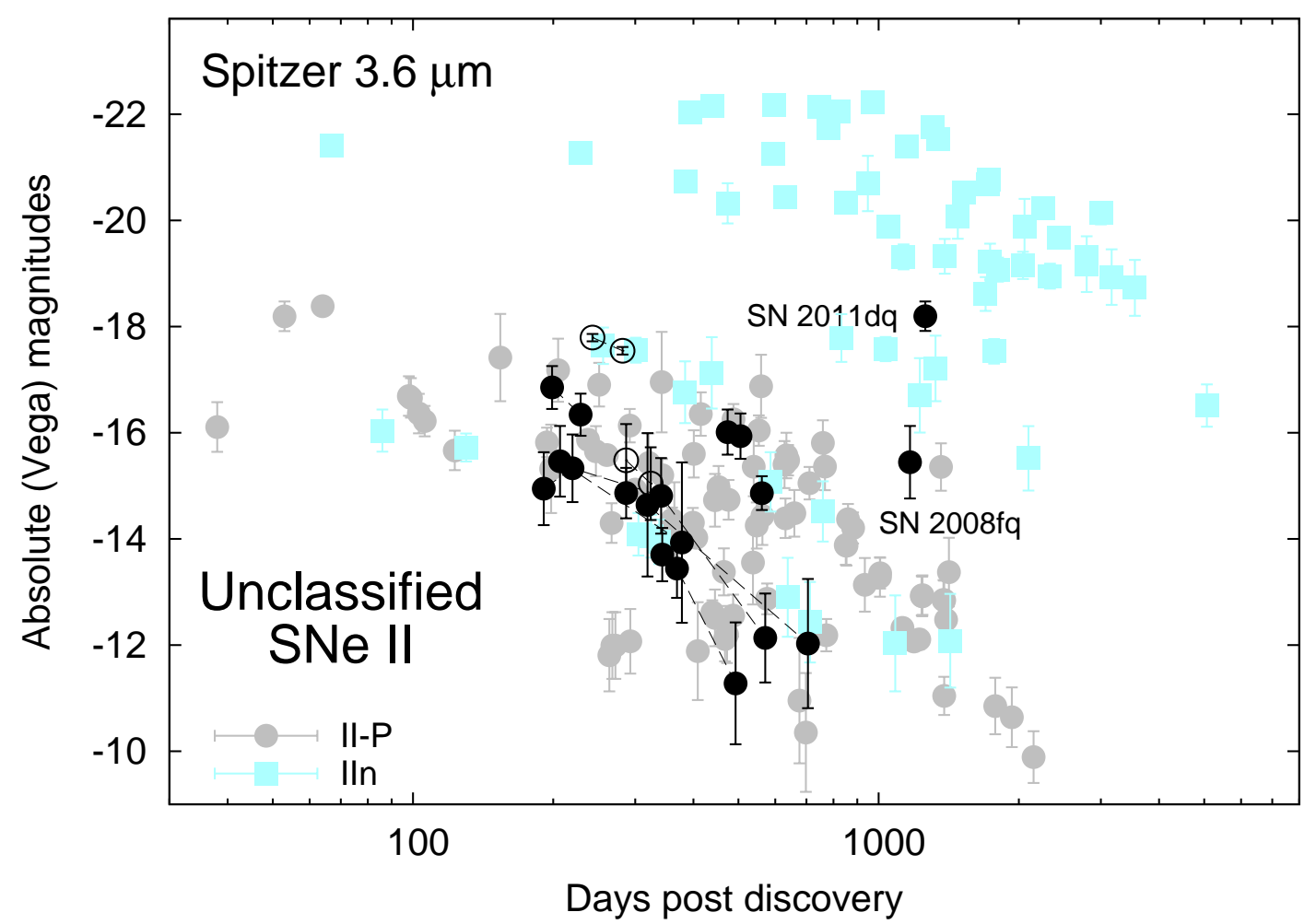

Figure 9. Mid-IR evolution of the unclassified Type II SNe in our sample (black symbols) compared to that of known SNe II-P and IIn (gray circles and rectangles, respectively). Filled and empty symbols denote SNe whose absolute magnitudes were determined with or without image subtraction, respectively (absolute magnitudes shown here are calculated from $3.6 \mu \mathrm{m}$ fluxes, because several objects have been observed only at this wavelength). Values and sources of data are shown in Table A3.

dened using the galactic reddening law parametrized by Fitzpatrick \& Massa (2007) assuming $\mathrm{R}_{V}=3.1$ and adopting $E(B-V)$ values listed in Table A1.

Fig. 10 shows that single component black bodies (BBs) provide a good fit to the combined optical-IR SEDs of both SNe. Fitting only the mid-IR data yields significantly different parameters (see Table 2, highlighting the shortcomings of fitting just two data points). Regardless, the SED in this case does not show any evidence for an excess of mid-IR emission above the optically peaked SED.

The Type Ib SN 2009jf also has sufficient data to construct a combined opticalIR SED adopting BVRI measurements from Sahu et al. (2011b). Spitzer data were obtained at 100 days after explosion, while optical data are from +94 and +105 days. Unlike SNe 2011A and 2014cx, Fig. 11 shows that SN 2009jf exhibits an excess at $4.5 \mu \mathrm{m}$, but not at $3.6 \mu \mathrm{m}$. Fitting the mid-IR data with a single BB is difficult.

Fig. 12 shows data for the Type II-P SN 2012aw. The earliest Spitzer observations occur on day 358, and we extrapolate V, R, and I-band data from day 330 (Dall'Ora et al. 2014). The hot component cannot be adequately modeled by a simple $\mathrm{BB}$ curve since the optical depth of the continuously expanding ejecta is quite low at this time. Therefore, we applied the global light-curve model of Type II-P SNe 

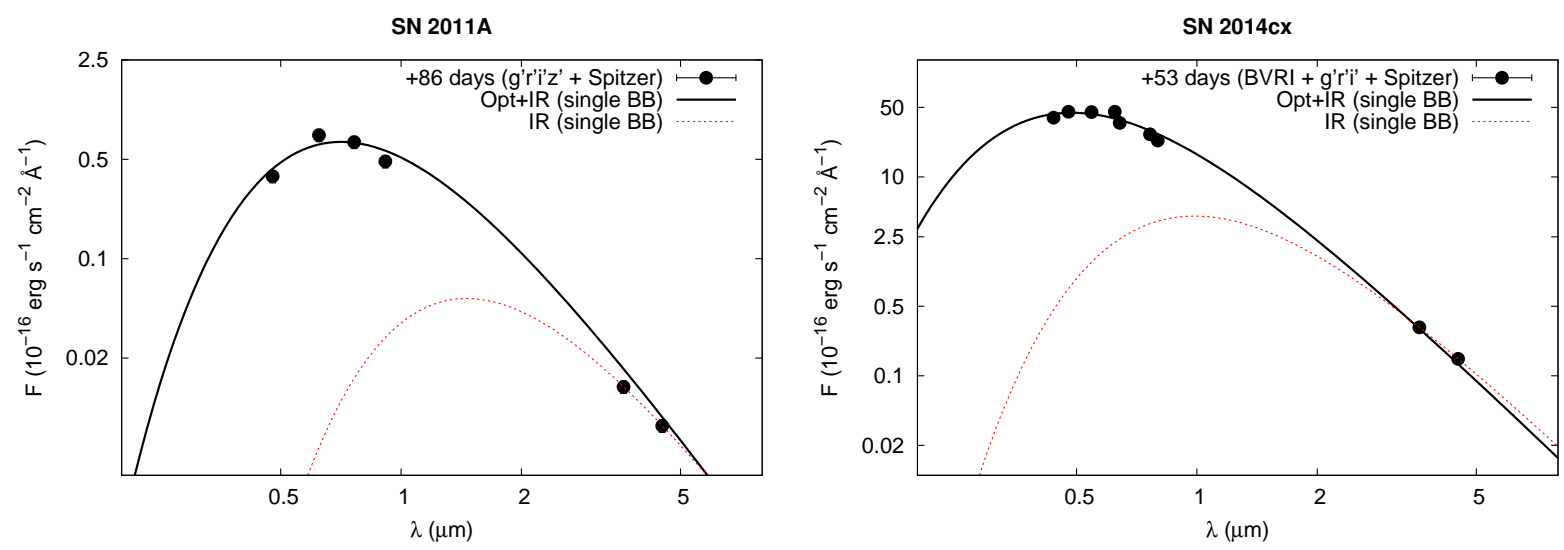

Figure 10. Comparison of single blackbody fits to the (left) Type IIn SN 2011A at +86 days, and (right) Type II-P SN 2014cx at +53 days. Fits are applied to both the combined optical-IR SEDs and only the mid-IR fluxes.

(Pejcha \& Prieto 2015, called hereafter PP15 model) to estimate the contribution of the hot component to the mid-IR fluxes. In order to construct the PP15 model SED, we calculated its values at the wavelengths of BVRIJHK filters, while, at longer wavelengths, we used the the Rayleigh-Jeans approximation $\left(\mathrm{F}_{\lambda} \propto \lambda^{-4}\right)$. Like SN 2009jf, there is an excess at $4.5 \mu \mathrm{m}$, indicating a warm dust component is present. Fitting this component is difficult with just two data points and complicated even further by the potential $4.5 \mu \mathrm{m}$ line emission in SNe II-P described above.

Finally, Fig. 12 shows a similar analysis for the CSM-interacting Type II-P/II-L SN 2013ej (Leonard et al. 2013; Bose et al. 2015; Kumar et al. 2016; Dhungana et al. 2016; Chakraborti et al. 2016; Mauerhan et al. 2017). Despite the amount of published data, modeling of the combined (UV)-optical-IR SEDs has not been presented in the literature. Only one epoch $(+236 \mathrm{~d})$, however, has nearly contemporaneous mid-IR and optical data (Tinyanont et al. 2016; Bose et al. 2015, respectively). Like SN 2012aw, we fit the optical data with the PP15 model with parameters given by Müller et al. (2017). We ignore the R-band data in this case, however, given the strong $\mathrm{H} \alpha$-emission arising from CSM interaction (Bose et al. 2015; Huang et al. 2015; Dhungana et al. 2016; Mauerhan et al. 2017). The results for each fit are given in Table 3.

We performed a similar analysis on the rest of the targets in our sample. Since we are most interested in late-time emission and want to minimize contributions from the early-time photospheric light-curve, we excluded targets that did not meet certain criteria. For example, we did not include SNe without late-time observations or only single filter IRAC photometry. We also exclude "normal" Type Ia SNe since their mid-IR photometry do not probe warm dust (but see Nozawa et al. 2011).

For the SNe we analyze, we follow the method published in a number of papers (see e.g. Meikle et al. 2007; Fox et al. 2010, 2011, 2016; Fox \& Filippenko 2013; Szalai \& Vinkó 2013; Graham et al. 2017) by assuming a spherically symmetric, optically thin dust shell. We calculate the minimum shell radius by fitting $\operatorname{BBs}\left(R_{B B}\right)$ 


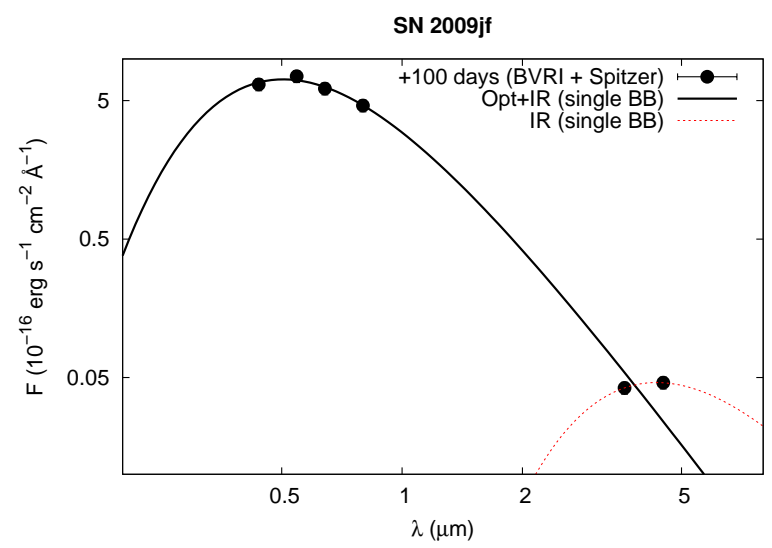

Figure 11. Comparison of single blackbody fits to the Type Ib SN 2009jf at +100 days. A fit is applied to both the combined optical-IR SEDs and only the mid-IR fluxes.
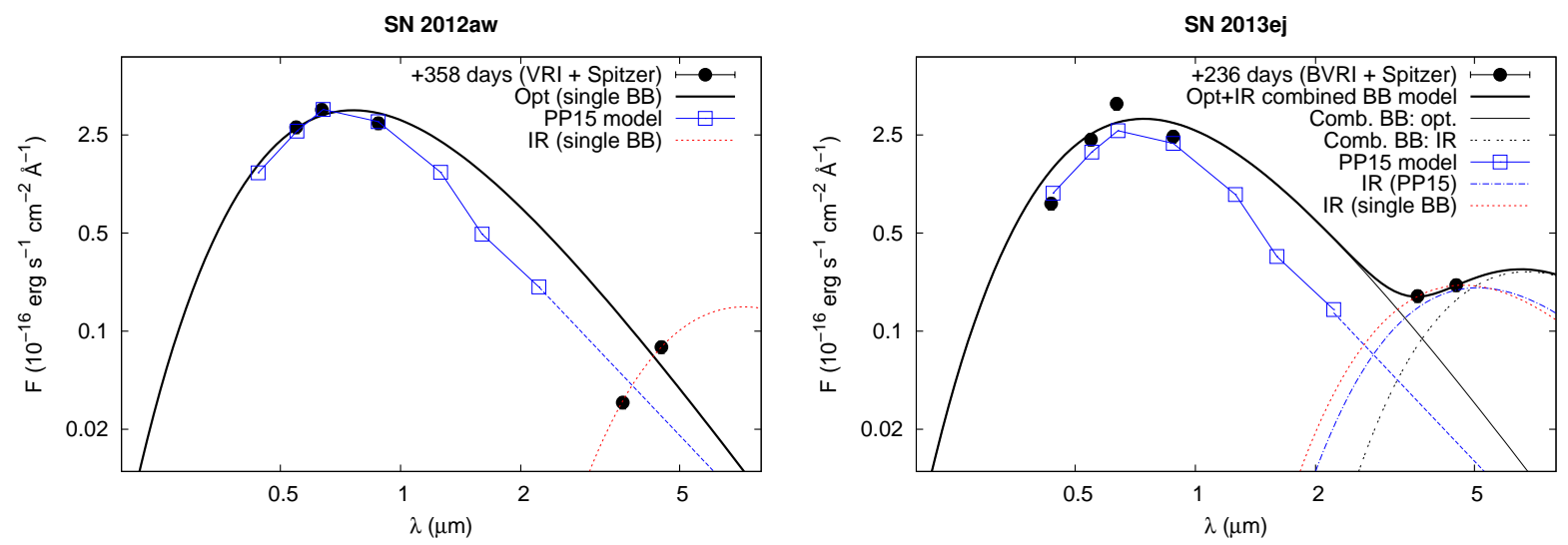

Figure 12. Comparison of single blackbody fits to the (left) SN 2012aw at +358 days and (right) SN 2013ej at +236 days. Fits are applied to both the combined optical-IR SEDs and only the mid-IR fluxes. SEDs calculated using the PP15 model are marked with open rectangles.

Table 2. Parameters of single BBs fitted to the optical-IR SEDs of SNe 2011A (IIn), 2014cx (II-P), 2009jf (Ib), and 2012aw (II-P).

\begin{tabular}{c|cc|cc|cc|cr}
\hline \hline & \multicolumn{2}{|c}{ SN 2011A } & \multicolumn{2}{c}{ SN 2014cx } & \multicolumn{2}{c}{ SN 2009jf } & \multicolumn{2}{c}{ SN 2012aw } \\
& \multicolumn{2}{c|}{$(\mathrm{IIn},+86 \mathrm{~d})$} & $(\mathrm{II}-\mathrm{P},+53 \mathrm{~d})$ & $(\mathrm{Ib},+100 \mathrm{~d})$ & \multicolumn{2}{c}{$(\mathrm{II}-\mathrm{P},+358 \mathrm{~d})$} \\
& $R$ & $T$ & $R$ & $T$ & $R$ & $T$ & $R$ & $T$ \\
& $\left(10^{16} \mathrm{~cm}\right)$ & $(\mathrm{K})$ & $\left(10^{16} \mathrm{~cm}\right)$ & $(\mathrm{K})$ & $\left(10^{16} \mathrm{~cm}\right)$ & $(\mathrm{K})$ & $\left(10^{16} \mathrm{~cm}\right)$ & $(\mathrm{K})$ \\
\hline Opt. + IR (single BB) & 0.07 & 4100 & 0.13 & 5960 & 0.09 & 5760 & 0.05 & 3810 \\
IR (single BB) & 0.12 & 1990 & 0.23 & 2940 & 1.48 & 670 & 2.79 & 400 \\
\hline
\end{tabular}

to the observed SEDs and, from the radii and the estimated ages, we also constrain the corresponding expansion velocities $\left(v_{B B}\right)$ by assuming a constant expansion over time (see Table A4). 
Table 3. Parameters of two- and one-component blackbodies fitted to the combined opticalIR SED of the known interacting Type II-P/II-L SN 2013ej, together with dust parameters determined from fitting a simply analytic dust model comparing with previously published results of Tinyanont et al. (2016).

\begin{tabular}{c|cccc|ccc}
\hline & \multicolumn{7}{|c}{ SN 2013ej (II-P/II-L, +236d) } \\
& $\begin{array}{c}R_{\text {opt }} \\
\left(10^{16} \mathrm{~cm}\right)\end{array}$ & $\begin{array}{c}T_{\text {opt }} \\
(\mathrm{K})\end{array}$ & $\begin{array}{c}R_{I R} \\
\left(10^{16} \mathrm{~cm}\right)\end{array}$ & $\begin{array}{c}T_{I R} \\
(\mathrm{~K})\end{array}$ & $\begin{array}{c}T_{\text {dust }} \\
(\mathrm{K})\end{array}$ & $\begin{array}{c}M_{\text {dust }} \\
\left(10^{-5} \mathrm{M}_{\odot}\right)\end{array}$ & $\begin{array}{c}L_{\text {dust }} \\
\left(10^{6} L_{\odot}\right)\end{array}$ \\
\hline Two-comp. BBs & 0.05 & 3910 & 3.48 & 430 & 360 & 580 & 5.3 \\
Opt. (PP15) + IR BB & - & - & 1.51 & 570 & 460 & 98.8 & 3.3 \\
IR (single BB) & - & - & 1.25 & 620 & 490 & 69.9 & 2.9 \\
\hline Tinyanont et al. (2016) - IR & - & - & - & - & 477 & 75.0 & 2.7 \\
\hline
\end{tabular}

For comparison, we also fit analytic dust model adopted from Fox et al. (2010, 2011), assuming only thermal emission of optically thin dust with mass $M_{d}$, with a particle radius $a$, at a distance $d$ from the observer, thermally emitting at a single equilibrium temperature $T_{d}$; hence, the flux can be written as

$$
F_{\nu}=\frac{M_{d} B_{\nu}\left(T_{d}\right) \kappa_{\nu}(a)}{d^{2}}
$$

where $B_{\nu}\left(T_{d}\right)$ is the Planck-function, $\kappa_{\nu}$ is the dust mass absorption coefficient as a function of the grain size. We chose pure graphite composition assuming single-sized grains of $a=0.1 \mu \mathrm{m}$ (following Fox et al. 2010, 2011). During the fit, only $T_{d}$ and $M_{d}$ are free parameters; $\kappa_{\nu}$ has been determined from Fig. 4 of Fox et al. (2010). In cases of two-point SEDs, we are limited to using one temperature component.

Figure 13 compares the analytical and blackbody fits in two SNe that have data from all four IRAC channels: the Type IIn SN 2002bu and and the Type Ib/c 2001em. SN 2002bu was observed $\sim 2$ years post-explosion and can be fit with just a singlecomponent graphite or blackbody dust model. SN 2001em, however, requires a twocomponent model. If we fit use blackbodies, we get the parameters shown in Figure 13. We can compare our results with those of Chugai \& Chevalier (2006) who constructed a model for the strong late-time X-ray, radio, and $\mathrm{H} \alpha$ emission from SN 2001em and developed a picture in which the SN ejecta collide with a dense massive CS shell. Our two-component model gives $\sim 10^{16} \mathrm{~cm}$ and $\sim 15 \times 10^{16} \mathrm{~cm}$ for the two radii, which is compatible with the estimated size of the single CS shell $\left(\mathrm{r} \sim 7 \times 10^{16} \mathrm{~cm}\right)$ calculated by Chugai \& Chevalier (2006) from X-ray, radio, and $\mathrm{H} \alpha$ data contemporaneous with mid-IR observations. If we change the longer-wavelength blackbody to a graphite dust model, we get $T_{d u s t}=280 \mathrm{~K}$ and an upper limit of $M_{d u s t} \approx 0.2 M_{\odot}$, which are in a good agreement with the calculations of Chugai \& Chevalier (2006) who derived indirectly $-300 \mathrm{~K}$ for dust temperature and 2-3 $M_{\odot}$ for the mass of the CS shell (which gives $0.02-0.03 M_{\odot}$ dust mass assuming a 0.01 dust-to-gas mass ratio). These results strengthen previous conclusions of CSM interaction with SN 2001em, but further suggest the presence of multiple pre-explosion dust shells. 

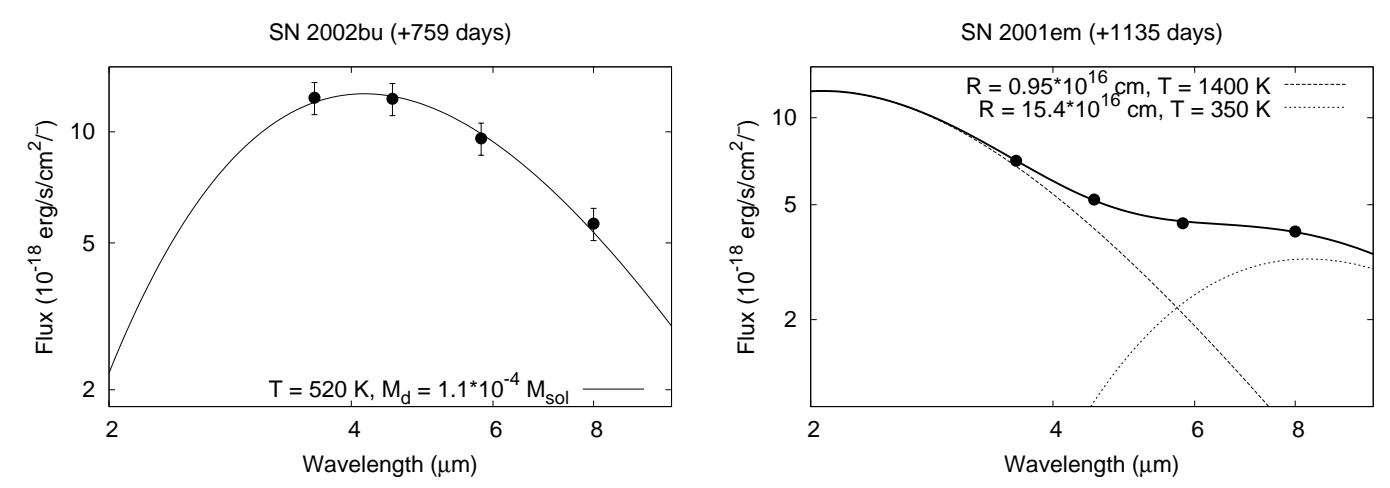

Figure 13. Left: One-component carbonaceous dust model fit to the four-point mid-IR SED of the Type IIn SN 2002bu. Right: Two-component blackbody model fit to the fourpoint mid-IR SED of the known interacting Type Ib/c SN 2001em.

Table A4 lists and Figures 14-17 plot the best fit dust parameters (masses, temperatures, mid-IR luminosities). We fitted SEDs of all listed SNe using the dust model described above in order to generate a comparable set of dust parameters. Nevertheless, in general, one cannot distinguish between dust compositions with only two IRAC filters. We include the results of silicate fits only in cases with spectroscopic evidence, such as SN 2004et (Kotak et al. 2009; Fabbri et al. 2011) or SN 2005af (Szalai \& Vinkó 2013). In some other cases, the temperature may provide guidance on the dust composition. For example, if $T_{\text {dust }} \gtrsim 1400 \mathrm{~K}$, then the carbonaceous dust model makes the most sense since Si grains require lower temperatures for effective condensation (see e.g. Nozawa et al. 2003).

Different dust models (composition, grain size) used in the literature result systematic uncertainties in dust parameters. After comparing our results with previously published ones, we draw the following conclusions: the uncertainties can be as large as $\sim 100-150 \mathrm{~K}$ in dust temperature (which is also significantly influenced by number of SED points including additional optical and/or near-IR data), while dust masses and dust luminosities can vary within one order of magnitude and within a factor of $\sim 1-2$, respectively. We also note that choosing non-spherical geometry for the dust-forming region, or, assuming clumpy dust formation (see e.g. Meikle et al. 2007; Ercolano et al. 2007; Andrews et al. 2016) may also lead to significantly (an order of magnitude lower/higher) different calculated dust masses.

While the SED fits in Table A4 have a number of uncertainties, we can still draw some useful conclusions. The blackbody expansion velocities $\left(v_{B B}\right)$, shown on the bottom right panels of Figs. 14-17, can distinguish between newly-formed and preexisting dust. In cases where $v_{B B}$ is quite low (several hundreds or a few thousands $\mathrm{km}$ $\mathrm{s}^{-1}$ ), the dust likely formed in the ejecta. In these cases, cover many Type II-P and SE CC SNe, we find the estimated temperatures and dust masses $\left(\sim 10^{-6}-10^{-2} M_{\odot}\right)$ are in agreement with this scenario (see e.g. Fox et al. 2011, 2013; Szalai \& Vinkó 2013; Tinyanont et al. 2016). 
Dust/BB parameters - thermonuclear SNe
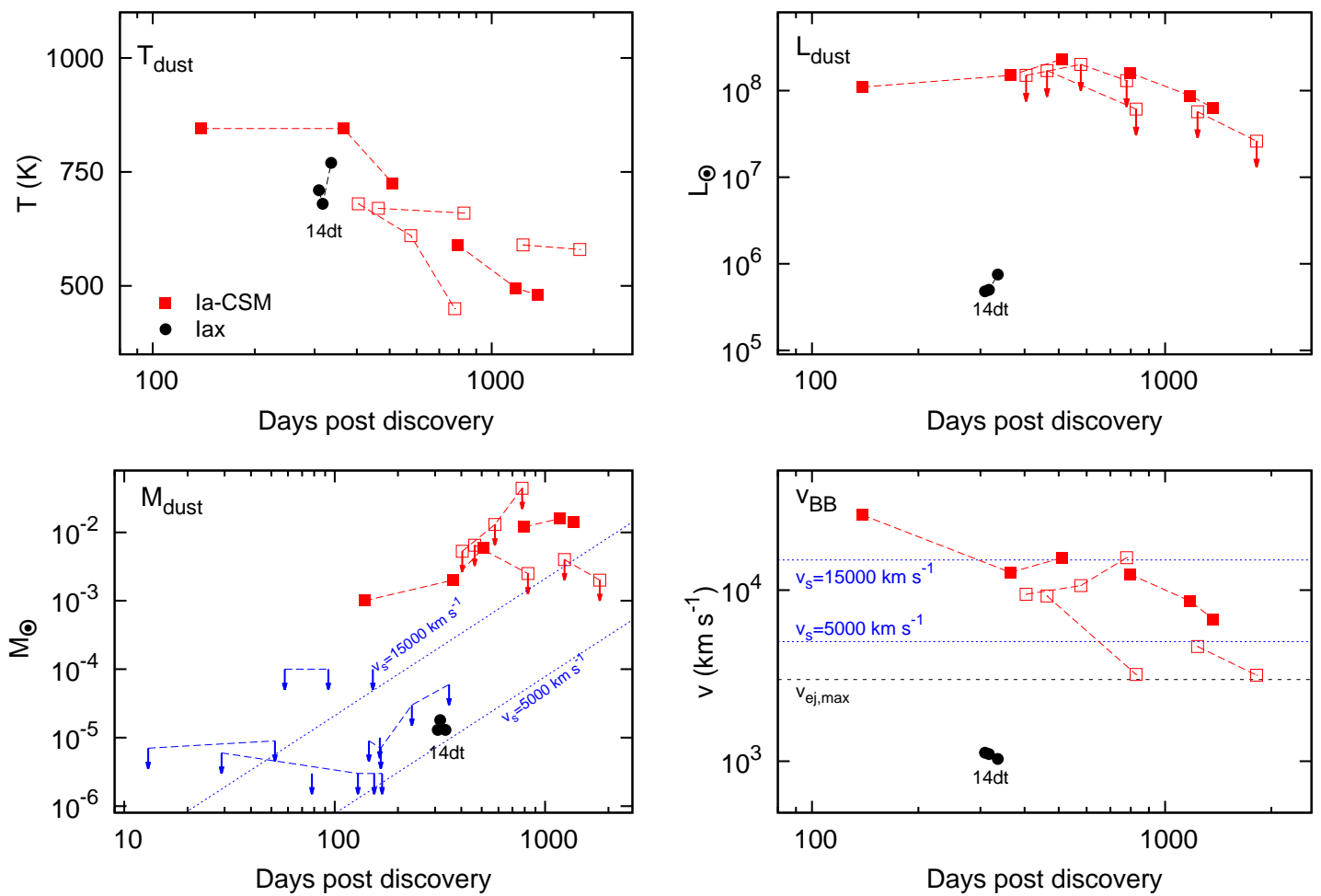

Figure 14. Dust parameters (temperatures - top left, luminosities - top right, and dust masses - bottom left) and blackbody velocities (belong to minimum dust radii) of thermonuclear SNe derived from the SED fits. Filled and empty symbols denote SNe whose absolute magnitudes were determined with or without image subtraction, respectively in the latter cases, only upper limits can be determined for dust masses and luminosities (marked with arrows on both bottom left and top right panels). Blue arrows denote upper dust mass limits for a group of SNe Ia calculated by Johansson et al. (2017). Dotted lines on the bottom left panel denote theoretical dust masses at shock velocities $v_{s}=5000$ and $15000 \mathrm{~km} \mathrm{~s}^{-1}$ assuming a shock heating scenario (see text for details); at the bottom right panel, the mentioned shock velocities are shown together with an upper limit of late-time ejecta velocities (black dashed line) expected in thermonuclear $\mathrm{SNe}\left(v_{e j, \max }=3000 \mathrm{~km} \mathrm{~s}^{-1}\right.$, based on Silverman et al. 2013).

In cases where $v_{B B}$ is a bit higher $\left(\sim 5000-15000 \mathrm{~km} \mathrm{~s}^{-1}\right)$, the velocities are consistent with the forward shock, suggesting new dust may be forming in the CDS behind the forward shock. Nevertheless, especially in the cases of SNe IIn or Ia-CSM, or other known interacting objects (e.g. SN Ib 2014C) with large $\left(>10^{-3} M_{\odot}\right)$ observed dust masses, the presence of pre-existing dust should be invoked for explaining the amount of the observed mid-IR luminosities. For the distinction between the collisional and radiative heating scenarios, we adopt the method presented in Fox et al. (2011) (and also used by e.g. Tinyanont et al. 2016). Eq. 2, assuming a dust to gas ratio of 0.01 , gives the mass of dust processed by the forward shock of the $\mathrm{SN}$ :

$$
M_{\mathrm{d}}\left(M_{\odot}\right) \approx 0.0028\left(\frac{v_{\mathrm{s}}}{15000 \mathrm{~km} \mathrm{~s}^{-1}}\right)^{3}\left(\frac{t}{\text { year }}\right)^{2}\left(\frac{a}{\mu \mathrm{m}}\right)
$$


Dust/BB parameters - SE CC SNe
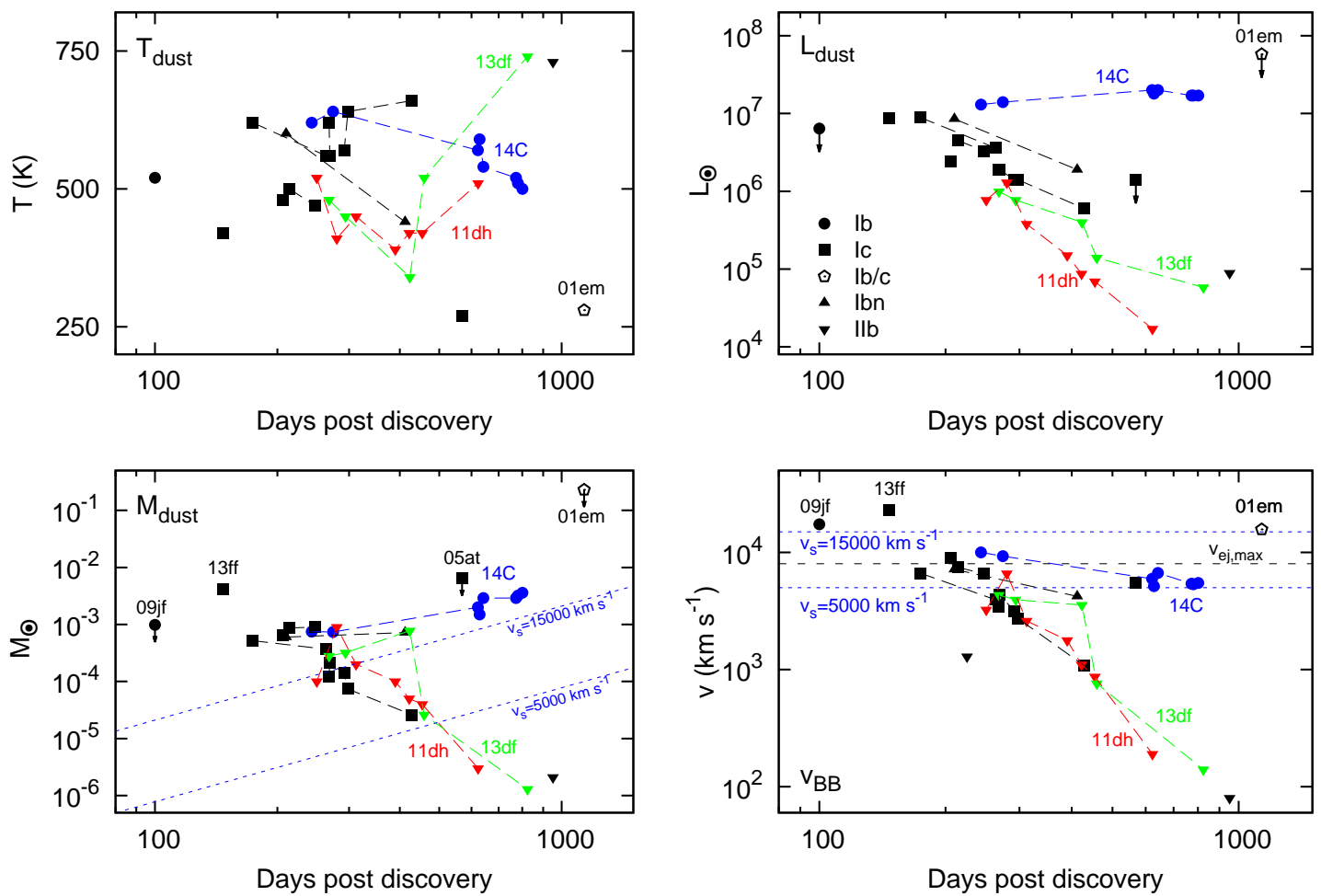

Figure 15. Same as Figure 14, except in this case for stripped-envelope CC SNe. Filled and empty symbols denote SNe whose absolute magnitudes were determined with or without image subtraction, respectively - in the latter cases (and in some other ones), only upper limits can be determined for dust masses and luminosities (marked with arrows on both bottom left and top right panels). At the bottom right panel, black dashed line denotes an upper limit of late-time ejecta velocities expected in SE CC SNe $\left(v_{e j, \max }=8000 \mathrm{~km} \mathrm{~s}^{-1}\right.$, based on Taubenberger et al. 2006).

where $v_{\mathrm{s}}$ is the shock velocity, $t$ is the time post explosion, and $a$ is the grain size (assumed to be $0.1 \mu \mathrm{m}$ ). The calculated dust masses - using $v_{\mathrm{s}}=5000 \mathrm{~km} \mathrm{~s}^{-1}$ and $15000 \mathrm{~km} \mathrm{~s}^{-1}$ for the shock velocities assumed to be constant - appear as straight lines on the bottom left panels of Figs. 14-17. A large fraction of Type IIn and other strongly interacting SNe show much larger dust masses than what can be expected even at $v_{\mathrm{s}}=15000 \mathrm{~km} \mathrm{~s}^{-1}$; in these cases, radiative heating by the photons may emerge from the ongoing CSM interaction.

Finally, in cases where $v_{B B}$ is very high (over $\sim 15000 \mathrm{~km} \mathrm{~s}^{-1}$ ), the dust is likely located beyond the forward shock, suggesting the dust is pre-existing at the time of the explosion and radiatively heated. Such high velocities can be seen mainly in earlytime (<1yr) observations, found e.g. in the cases of Type IIn SN 2010jl (Gall et al. 2014; Fransson et al. 2014) or Type II-P SN 2014bi (Tinyanont et al. 2016). In these cases, another possible source of mid-IR emission may be an IR echo in which the dust shell is heated by the peak luminosity of the SN (e.g. Bode \& Evans 1980; Dwek 

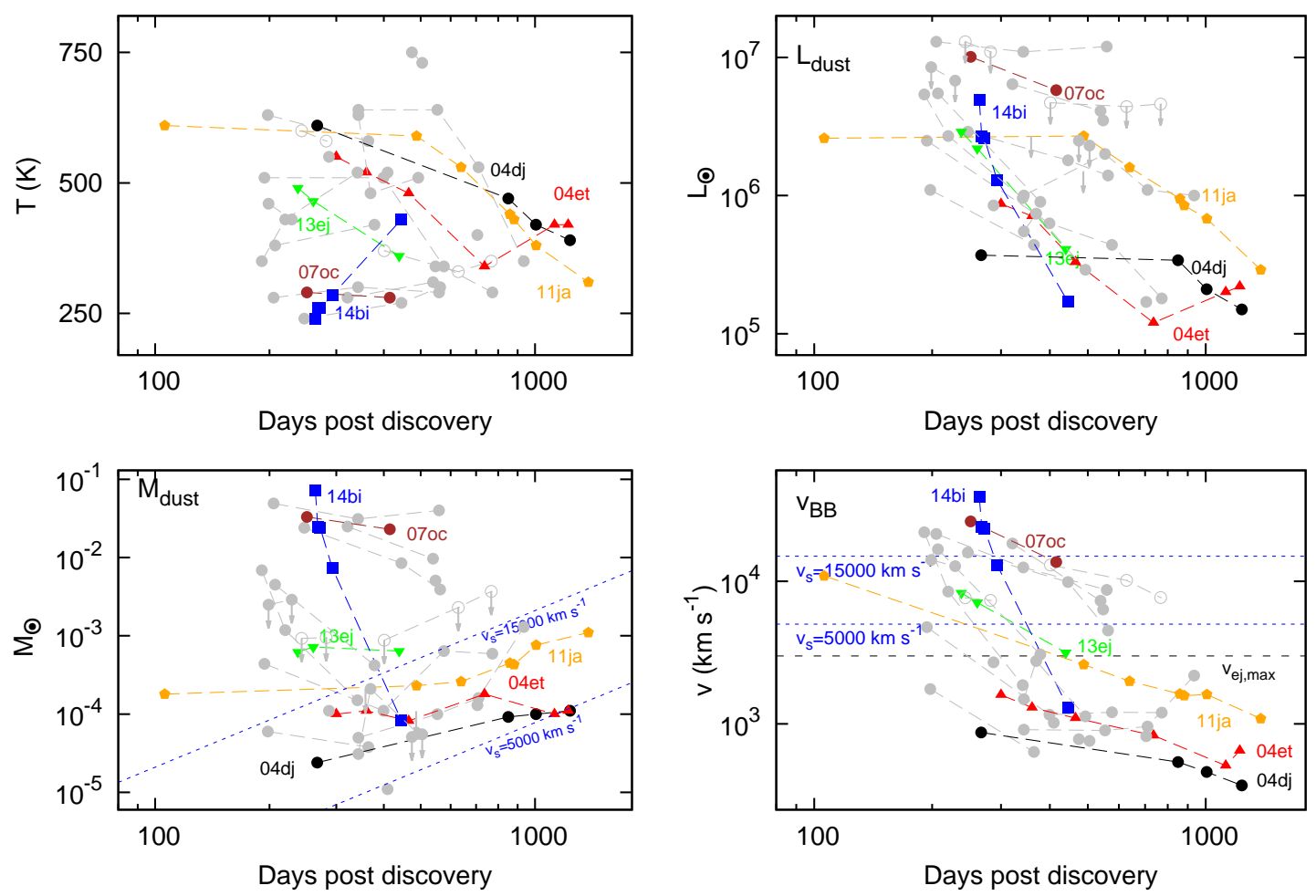

Figure 16. Same as Figure 14, except in this case for SNe II-P. Filled and empty symbols denote SNe whose absolute magnitudes were determined with or without image subtraction, respectively - in the latter cases (and in some other ones), only upper limits can be determined for dust masses and luminosities (marked with arrows on both bottom left and top right panels). At the bottom right panel, black dashed line denotes an upper limit of late-time ejecta velocities expected in SNe II-P $\left(v_{e j, \max }=3000 \mathrm{~km} \mathrm{~s}^{-1}\right.$, based on Szalai et al. 2011).

1983; Sugerman 2003; Meikle et al. 2006). At later epochs, however, this possibility probably can be ruled out (see details in Fox et al. 2011).

\section{CONCLUSION}

Here we presented a comprehensive study of all SNe (discovered before 2015) observed with Spitzer/IRAC, both targeted and untargeted. In total, we increased the published sample of Spitzer SNe by a factor of $\sim 5 \times$ (from $\sim 200$ to $\sim 1100$ ), including nearly $\sim 2 \times$ more detections $(\sim 70$ to $\sim 120)$.

We carry out a thorough photometric analysis of the entire SN sample, including all previously published data. In general, we find good agreements with the published values ( $\lesssim 10 \%$ difference in fluxes), except in some cases that were captured in a very faint phase and/or with a complex sky background (however, for this reason, the uncertainties of their original fluxes are also implicitly large).

The results include both a detailed analysis of specific targets with unique behavior and a statistical analysis of the mid-IR evolution of the different SN subclasses. For each detection, we fit both black bodies and simple analytic dust models. Modeling 
Dust/BB parameters - SNe IIn
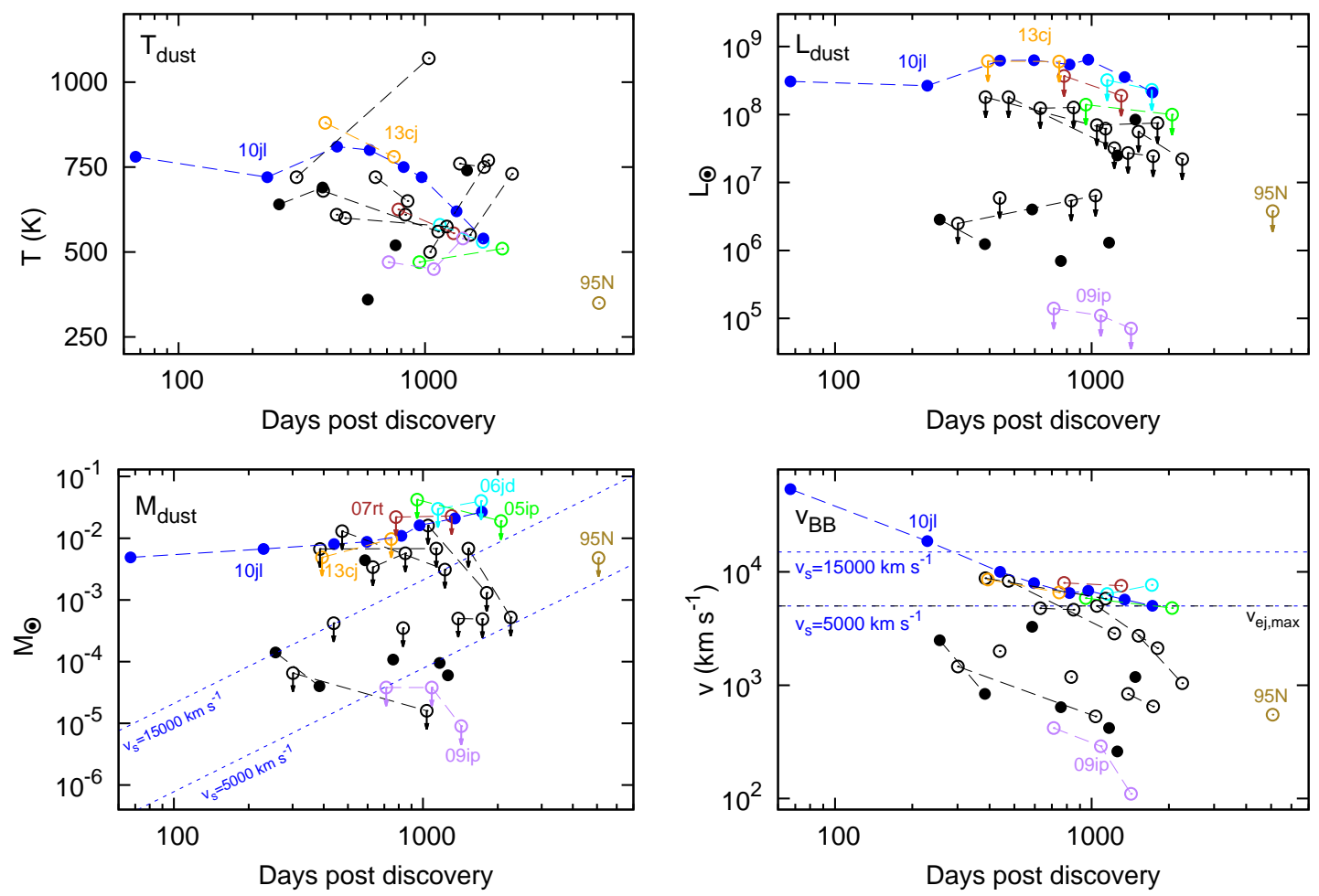

Figure 17. Same as Figure 14, except in this case for SNe IIn. Filled and empty symbols denote SNe whose absolute magnitudes were determined with or without image subtraction, respectively - in the latter cases, only upper limits can be determined for dust masses and luminosities (marked with arrows on both bottom left and top right panels). At the bottom right panel, black dashed line denotes an upper limit of late-time ejecta velocities expected in SNe IIn $\left(v_{e j, \max }=5000 \mathrm{~km} \mathrm{~s}^{-1}\right.$, based on Patat et al. 2001).

the SEDs (even in cases with just two photometry points) can disentangle the dust origin and heating mechanism, and, in some cases, to determine the main physical parameters of the assumed dust. Large dust masses $\left(\gtrsim 10^{-3} M_{\odot}\right)$ are observed primarily in Type IIn and other strongly interacting SNe. The associated $v_{B B}$ is quite high in most of these cases, again consistent with pre-existing, radiatively heated grains.

The large data set allows us to draw some broad conclusions; nevertheless, we note that these are based on studying a quite heterogeneous sample with usually 1-2 epochs of data per object. In general, each subclass tends to fill its own region of phase space. Amongst thermonuclear explosions (looking over the late-time mid-IR data of several hundred objects, finding mostly non-detections), we see that i) SNe Ia-CSM may be rare indeed, and ii) only a very limited number of "intermediate" cases with moderately strong CSM interaction may exist (suggested by a $~ 8-10$ mag gap in latetime mid-IR brightness of strongly interacting and slightly- or nondetected objects). Secondly, in the heterogeneous group of stripped-envelope CC SNe, the length of midIR light-curve seems to correlate with the assumed size of the progenitor (the larger the progenitor was, the longer the mid-IR light-curve is, from Type eIIb SNe to Type 
cIIb and Type Ib/c ones); however, this finding is based on a not-so-large sample of objects. Finally, Type IIn SNe may remain bright for several years post-explosion or may fade more quickly.

Although this study has significantly enlarged the sample sizes for all subclasses, however, the cadence is quite under-sampled both spectrally and temporally. Future observations with the James Webb Space Telescope (JWST) offer the sensitivity and spectral capabilities necessary to further constrain the dust geometry, mass, temperature, and composition.

We thank our anonymous referee for his/her valuable comments. This work is part of the project "Transient Astrophysical Objects" GINOP-2-3-2-15-2016-00033 of the National Research, Development and Innovation Office (NKFIH), Hungary, funded by the European Union, and is also supported by the New National Excellence Program (UNKP-17-2, UNKP-17-4) of the Ministry of Human Capacities of Hungary. TS has received funding from the Hungarian NKFIH/OTKA PD-112325 Grant. OP is currently supported by award PRIMUS/SCI/17 from Charles University. TM was supported in part by the Ministry of Economy, Development, and Tourisms Millennium Science Initiative through grant IC120009, awarded to the Millennium Institute of Astrophysics, MAS. TM thanks the LSSTC Data Science Fellowship Program, his time as a Fellow has benefited this work. TM was funded by the CONICYT PFCHA/DOCTORADO BECAS CHILE/2017 - 72180113.

This research has made use of the NASA/IPAC Infrared Science Archive, which is operated by the Jet Propulsion Laboratory, California Institute of Technology, under contract with the National Aeronautics and Space Administration; the NASA/IPAC Extragalactic Database (NED), which is operated by the Jet Propulsion Laboratory, California Institute of Technology, under contract with the National Aeronautics and Space Administration; and the SIMBAD database, operated at CDS, Strasbourg, France. This publication makes use of data products from the Two Micron All Sky Survey, which is a joint project of the University of Massachusetts and the Infrared Processing and Analysis Center/California Institute of Technology, funded by the National Aeronautics and Space Administration and the National Science Foundation. We acknowledge the availability of NASA ADS services.

Software: IRAF, HOTPANTS

\section{REFERENCES}

Andrews, J. E., Gallagher, J. S., Clayton, G. C., et al. 2010, ApJ, 715, 541

Andrews, J. E., Clayton, G. C., Wesson, R., et al. 2011a, AJ, 142, 45
Andrews, J. E., Sugerman, B. E. K., Clayton, G. C., et al. 2011b, ApJ, 731, 47

Andrews, J. E., Smith, N., Fong, W.-F., \& Milne, P. 2015, ATel, 7084, 1 
Andrews, J. E., Krafton, K. M., Clayton, G. C., et al. 2016, MNRAS, 457, 3241

Andrews, J. E., Smith, N., McCully, C., et al. 2017, MNRAS, 471, 4047

Arbour, R., \& Briggs, D. 2009, CBET, 1964, 1

Balanutsa, P., Parhomenko, A. V., Tlatov, A., et al. 2011, ATel, 3610, 1

Barna, B., Szalai, T., Kromer, M., et al., 2017, MNRAS, 471, 4865

Benetti, S.,Valenti, S., Magazzu, A., \& Harutyunyan, A. 2009, CBET, 1667, 1

Bietenholz, M. F., Bartel, N. 2005, ApJ, 625, L99

Blackman, J., Schmidt, B., \& Kerzendorf, W. 2006, CBET, 541, 1

Bode, M. F., \& Evans, A. 1980, MNRAS, 193, 21

Boles, T. 2009, CBET, 1648, 1

Boles, T. 2011, CBET, 2851, 1

Bose, S., Sutaria, F., Kumar, B., et al. 2015, ApJ, 806, 160

Brimacombe, J., Zaggia, S., Barbieri, M., et al. 2013, CBET, 3647, 1

Burket, J., \& Li, W. 2005, IAU Circ. 8472, 1

Casper, C., Zheng, W., Li, W., Filippenko, A. V., \& Cenko, S. B. 2013, CBET, 3588, 1

Chakraborti, S., Ray, A., Smith, R., et al. 2016, ApJ, 817, 22

Chen, J., Wang, X.-F., Wang, X.-L., et al. 2011, CBET, 2943, 2

Chevalier, R. A., \& Soderberg, A. M., 2010, ApJ, 711, L40

Chomiuk, L., Soderberg, A. M., Chevalier, R. A., et al. 2016, ApJ, 821, 119

Chornock, R., \& Berger, E. 2009, CBET, 2086, 1

Chugai, N. N., \& Chevalier, R. A. 2006, ApJ, 641, 1051

Ciroi, S., di Mille, F., Carco, M., et al. 2009, CBET, 1697, 2

Corsi, A., Ofek, E. O., Gal-Yam, A., et al. 2014, ApJ, 782, 42

Cortini, G. 2009, CBET, 1697, 1

Cortini, G., Brimacombe, J., Tomasella, L., et al. 2013, CBET, 3597, 1

Dall'Ora, M., Botticella, M. T., Pumo, M. L., et al. 2014, ApJ, 787, 139 de Jaeger, T., Anderson, J. P., Pignata, G., et al. 2015, ApJ, 807, 63

Dhungana, G., Kehoe, R., Vinkó, J., et al. 2016, ApJ, 822, 6

Drake, A. J., Djorgovski, S. G., Graham, M. J., et al. 2013, CBET, 3570, 1

Drescher, C., Parker, S., \& Brimacombe, J. 2012, CBET, 3101, 1

Dwek, E. 1983, ApJ, 274, 175

Dwek, E., Arendt, R. G., Bouchet, P., et al. 2010, ApJ, 722, 425

Elias-Rosa, N., Navasardyan, H., Harutunyan, A., et al. 2005, IAU Circ., 8479, 3

Ercolano, B., Barlow, M. J., \& Sugerman, B. E. K. 2007, MNRAS, 375, 753

Ergon, M., Jerkstrand, A., Sollerman, J., et al. 2015, A\&A, 580, 142

Fabbri, J., Otsuka, M., Barlow, M. J., et al. 2011, MNRAS, 418, 1285

Filippenko, A. V., Matheson, T., Ho, L. C. 1993, ApJL, 415, L103

Fitzpatrick, E. L., \& Massa, D. 2007, ApJ, 663, 320

Folatelli, G., Gutierrez, C., \& Pignata, G. 2010, CBET, 2390, 1

Foley, R. J. 2009, CBET, 1858, 1

Foley, R. J. 2010, CBET, 2137, 1

Foley, R. J., Challis, P. J., Chornock, R., et al., 2013, ApJ, 767, 57

Foley, R. J., Jha, S. W., Pan, Y-C., et al. 2016, MNRAS, 461, 433

Fox, O. D., \& Filippenko, A. V. 2013, ApJ, 772, L6

Fox, O. D., Chevalier, R. A., Dwek, E., et al. 2010, ApJ, 725, 1768

Fox, O. D., Chevalier, R. A., Skrutskie, M. F., et al. 2011, ApJ, 741, 7

Fox, O. D., Filippenko, A. V., Skrutskie, M. F., et al. 2013, AJ, 146, 2

Fox, O. D., Silverman, J. M., Filippenko, A. V., et al. 2015, MNRAS, 447, 772

Fox, O. D., Johansson, J., Kasliwal, M., et al. 2016, ApJ, 2016, 816, L13

Fransson, C., Ergon, M., Challis, P., et al. 2014, ApJ, 797, 118

Fraser, M., Inserra, C., Jerkstrand, A., et al. 2013, MNRAS, 433, 1332

Fraser, M., Kotak, R., Pastorello, A., et al. 2015, MNRAS, 453, 3886 
Gall, C., Andersen, A. C., \& Hjorth, J., 2011, A\&A, 528, A13

Gall, C., Hjorth, J., Watson, D., et al. 2014, Nature, 511, 326

Gallagher, J. S., Sugerman, B. E. K., Clayton, G. C., et al. 2012, ApJ, 753, 109

Gandhi, P., Yamanaka, M., Tanaka, M., et al. 2013, ApJ, 767, 166

Gerardy, C. L., Fesen, R. A., Nomoto, K., et al. 2002, ApJ, 575, 1007

Gerardy, C. L., Meikle, W. P. S., Kotak, R., et al. 2007, ApJ, 661, 995

Guillochon, J., Parrent, J., Kelley, L. Z., \& Margutti, R. 2017, ApJ, 835, 15

Graham, J., \& Li, W. 2005, IAU Circ., 8459, 3

Graham, J. R. \& Meikle, W. P. S. 1986, MNRAS, 221, 789

Graham, M. L., Harris, C. E., Fox, O. D., et al. 2017, ApJ, 843, 102

Harutyunyan, A., Bufano, F., \& Benetti, S. 2009, CBET, 1722, 1

Helou, G., Kasliwal, M. M., Ofek E. O., et al., 2013, ApJ, 778, L19

Holoien, T. W.-S., Prieto, J. L., Kochanek, C. S., et al. 2014, ATel, 6436, 1

Holoien, T. W.-S., Stanek, K. Z., Kochanek, C. S., et al. 2017a, MNRAS, 464, 2672

Holoien, T. W.-S., Brown, J. S., Stanek, K. Z., et al. 2017b, MNRAS, 467, 1098

Holoien, T. W.-S., Brown, J. S., Stanek, K. Z., et al. 2017c, MNRAS, 471, 4966

Howell, D. A., Murray, D. 2010, CBET, 3313, 2

Howerton, S., Drake, A. J., Djorgovski, S. G., et al. 2011, CBET, 2658, 1

Huang, F., Wang, X., Zhang, J., et al. 2015, ApJ, 807, 59

Huang, F., Wang, X., Zampieri, L., et al. 2016, ApJ, 832, 139

Indebetouw, R., Matsuura, M., Dwek, E., et al. 2014, ApJ, 782, L2

Inserra, C., Fraser, M., Smartt, S. J., et al. 2016, MNRAS, 459, 2721

Itagaki, K., Brimacombe, J., Noguchi, T., \& Nakano, S. 2011, CBET, 2943, 1
Jencson, J. E., Kasliwal, M. M., Johansson, J., et al. 2017, ApJ, 837, 167

Jencson, J. E., Kasliwal, M. M., Adams, S. M., et al. 2018, ApJ, 863, 20

Jha, S., Branch, D., Chornock, R., et al. 2006, AJ, 132, 189

Jin, Z., Gao, X., McCully, C., \& Jha, S. W. 2013, CBET, 3520, 1

Johansson, J., Goobar, A., Kasliwal, M. M., et al. 2017, MNRAS, 466, 3442

Kamble, A., Margutti, R., Soderberg, A. M., et al., 2016, ApJ, 818, 111

Kankare, E., Fraser, M., Ryder, S., et al. 2014, A\&A, 572, 75

Kasliwal, M. M. 2010, ATel, 2379, 1

Kasliwal, M. M., Howell, J. L., Fox, D., Quimby, R., \& Gal-Yam, A. 2009, ATel, 2218, 1

Kasliwal, M. M., Bally, J., Masci, F., et al. 2017, ApJ, 839, 88

Kochanek, C. S. 2017, MNRAS, 471, 3283

Kochanek, C. S., Szczygiel, D. M., \& Stanek, K. Z. 2011, ApJ, 737, 76

Kochanek, C. S., Szczygiel, D. M., \& Stanek, K. Z. 2012, ApJ, 758, 142

Kotak, R., Meikle, W. P. S., van Dyk, S. D., Höflich, P. A., \& Mattila, S. 2005, ApJ, 628, L123

Kotak, R., Meikle, W. P. S., Pozzo, M., et al. 2006, ApJ, 651, L117

Kotak, R., Meikle, W. P. S., Farrah, D., et al. 2009, ApJ, 704, 306

Kozasa, T., Nozawa, T., Tominaga, N., et al. 2009, in Cosmic Dust - Near and Far, ed. T. Henning, E. Grün, \& J. Steinacker (San Francisco: ASP), ASP Conf. Ser., 414, 43

Kumar, B., Pandey, S. B., Eswaraiah, C., \& Kawabata, K. S. 2016, MNRAS, 456, 3157

Lennarz, D., Altmann, D., \& Wiebusch, C. 2012, A\&A, 538, A120

Leonard, D. C., Pignata, G., Dessart, L., et al. 2013, ATel, 5275

Li, W., Foley, R. J., \& Filippenko, A. V. 2004, IAU Circ., 8345, 4

Li, W., Cenko, S. B., \& Filippenko, A. V. 2009a, CBET, 1656, 1

Li, W., Cenko, S. B., \& Filippenko, A. V. 2009b, CBET, 1952, 1 
Maeda, K., Nozawa, T., Nagao, T., Motohara, K. 2015, MNRAS, 452, 3281

Maeda, K., Hattori, T., Milisavljevic, D., et al., 2015, ApJ, 807, 35

Margutti, R., Milisavljevic, D., Soderberg, A. M., et al. 2014, ApJ, 780, 21

Margutti, R., Kamble, A., Milisavljevic, D., et al. 2017, ApJ, 835, 140

Marion, G. H., Foley, R. J., \& Calkins, M. 2012, CBET, 3106, 2

Marples, P., \& Drescher, C. 2009, CBET, 2080, 1

Marples, P., Drescher, C., Quirk, S., \& Bock, G. 2009, CBET, 1856, 1

Matheson, T., Jha, S., Challis, P.. Kirshner, R., \& Calkins, M. 2001, IAU Circ., 7761, 2

Matsuura, M., Dwek, E., Meixner, M., et al. 2011, Science, 333, 1258

Matsuura, M., Dwek, E., Barlow, M. J., et al. 2015, ApJ, 800, 50

Mattila, S., Meikle, W. P. S., Lundqvist, P., et al. 2008, MNRAS, 389, 141

Mauerhan, J. C., Smith, N., Filippenko, A. V., et al. 2013, MNRAS, 430, 1801

Mauerhan, J. C., Van Dyk, S. D., Johansson, J., et al. 2017, ApJ, 834, 118

Maza, J., Hamuy, M., Antezana, R., et al. 2009, CBET, 1937, 1

Maza, J., Hamuy, M., Antezana, R., et al. 2010a, CBET, 2125, 1

Maza, J., Hamuy, M., Antezana, R., et al. 2010b, CBET, 2388, 1

McClelland, C. M., Garnavich, P. M., Milne, P. A., et al. 2013, ApJ, 767, 119

McCully, C., Jha, S. W., Foley, R. J., et al., 2014, Nature, 512, 54

Meikle, W. P., Farrah, D., Fesen, R., et al. 2005, Spitzer Proposal, 20256

Meikle, W. P. S., Mattila, S., Gerardy, C. L., et al. 2006, ApJ, 649, 332

Meikle, W. P. S., Mattila, S., Pastorello, A., et al. 2007, ApJ, 665, 608

Meikle, W. P. S., Kotak, R., Farrah, D., et al. 2011, ApJ, 732, 109

Meng, X., Podsiadlowski, Ph. 2018, ApJ, 861,127

Milisavljevic, D., Margutti, R., Kamble, A., et al. 2015, ApJ, 815, 120
Modjaz, M., Kirshner, R., Challis, P., et al. 2005, IAU Circ., 8461, 2

Monard, L. A. G. 2009, CBET, 1798, 1

Monard, L. A. G., \& Li, W. 2002, IAU

Circ., 7940, 1

Monard, L. A. G., \& Prieto, J. L. 2011, CBET, 2759, 1

Monard, L. A. G., Prieto, J. L., \& Seth, K. 2011a, CBET, 2799, 1

Monard, L. A. G., Valenti, S., \& Benetti, S., 2011b, CBET, 2749, 1

Moore, M., Li, W., Filippenko, A. V.,

Chornock, R., \& Foley, R. J., 2004, IAU

Circ., 8286, 2

Morrell, N., \& Phillips, M. M. 2009, CBET, 1953, 1

Morrell, N., Folatelli, G., Hamuy, M., \& Phillips, M. M. 2006, CBET, 368, 1 Morrell, N., Hsiao, E. Y., Contreras, C., \& Phillips, M. M. 2013, ATel, 4707, 1 Müller, T., Prieto, J. L., Pejcha, O., \& Clocchiatti, A. 2017, ApJ, 841, 127

Nagao, T., Maeda, K., Yamanaka, M. 2017, ApJ, 835, 143

Nakano, S., Kushida, R.. Kushida, Y., \& Itagaki, K. 2004a, IAU Circ., 8272, 1

Nakano, S., Kushida, R., \& Kushida, Y. 2004b, IAU Circ., 8344, 1

Nakano, S., Hirose, Y., \& Li, W. 2005, IAU Circ., 8475, 1

Nakano, S., Kadota, K., Ikari, Y., \& Itagaki, K. 2009, CBET, 1718, 1

Nakano, S., Kadota, K., \& Itagaki, K. 2010, CBET, 2115, 1

Nakano, S., Yusa, T., Yoshimoto, K., et al. 2012, CBET, 3263, 1

Navasardyan, H., \& Benetti, S. 2009, CBET, 1806, 1

Navasardyan, H., Benetti, S., Bufano, F., \& Pastorello, A. 2009, CBET, 1738, 1

Nozawa, T., Kozasa, T., Umeda, H., Maeda, K., Nomoto, K. 2003, ApJ, 598, 785

Nozawa, T., Maeda, K., Kozasa, T., et al. 2011, ApJ, 736, 45

Ochner, P., Siviero, A., Tomasella, L., et al. 2014 ATel, 5767, 1

Ofek, E. O., Sullivan, M., Cenko, S. B., et al. 2013, Nature, 494, 65 
Papenkova, M., Li, W. D., Wray, J., Chleborad, C. W., \& Schwartz, M. 2011, IAU Circ., 7722, 1

Pastorello, A., Cappellaro, E., Inserra, C., et al. 2013, ApJ, 767, 1

Patat, F., Cappellaro, E., Danziger, J., et al. 2001, ApJ, 555, 900

Pejcha, O., \& Prieto, J. L. 2015, ApJ, 806, 225

Phillips, M. M., Simon, J. D., Morrell, N., et al. 2013, ApJ, 779, 38

Pignata, G., Cifuentes, M., Maza, J., et al. 2011, CBET, 2623, 1

Ponticello, N. J., Khandrika, H., Madison, D. R., et al. 2006, IAU Circ., 8709, 1

Pooley, D., \& Lewin, W. H. G. 2004, IAU Circ., 8323, 2

Pozzo, M., Meikle, W.P.S., Fassia, A., Geballe, T., Lundqvist, P., Chugai, N. N., Sollerman, J. 2004, MNRAS, 352, 457

Prieto, J. L. 2011a, CBET, 2759, 2

Prieto, J. L. 2011b, ATel, 3615, 1

Prieto, J. L., Seth, K. 2011, CBET, 2799, 2

Prieto, J. L., McMillan, R., Bakos, G., Grennan, D. 2011, CBET, 2903, 1

Prieto, J. L., Lee, J. C., Drake, A. J., et al. 2012, ApJ, 745, 70

Puckett, T., Reddy, V., \& Li, W. 2006 CBET, 363, 1

Quinn, J., Baade, D., Clocchiatti, A., et al. 2008, CBET, 1510, 1

Reach, W. T, Rho, J., Tappe, A., et al. 2006, AJ, 131, 1479

Sahu, D. K., Arora, S., \& Anto, P. 2011a, CBET, 2658, 2

Sahu, D. K., Gurugubelli, U. K., Anupama, G. C., \& Nomoto, K. 2011b, MNRAS, 413, 2583

Salvo, M., \& Price, P. 2002, IAU Circ., 7947, 2

Sanders, N. E., Soderberg, A. M., Foley, R. J., et al. 2013, ApJ, 769, 39

Shappee, B. J., Prieto, J. L., Grupe, D., et al. 2014, ApJ, 788, 48

Shurpakov, S., Balanutsa, P., Lipunov, V., et al. 2012, ATel, 4104, 1

Silverman, J. M., Kandrashoff, M. T., \& Filippenko, A. V. 2009, CBET, 1968, 1
Silverman, J. M., Nugent, P. E., Gal-Yam, A., et al. 2013, ApJS, 207, 3

Siviero, A., Tomasella, L., Pastorello, A., et al. 2012, CBET, 3054, 4

Skrutskie, M. F., Cutri, R. M., Stiening, R., et al. 2006, AJ, 131, 1163

Smith, N., Silverman, J. M., Chornock, R., et al. 2009, ApJ, 695, 1334

Soderberg, A. M., Gal-Yam, A., \& Kulkarni, S. R. 2004, GRB C. N., 2586, 1

Stockdale, C. J., Weiler, K. W., van Dyk, S. D., et al. 2004, IAU Circ., 8282, 2

Stritzinger, M., Prieto, J. L., Morrell, N., \& Pignata, G. 2011, CBET, 2623, 2

Stritzinger, M., Taddia, F., Fransson, C., et al. 2012, ApJ, 756, 173

Sugerman, B. E. K. 2003, AJ, 126, 1939

Sugerman, B. E. K., Meixner, M., Fabbri, J., \& Barlow, M. 2004, IAU Circ., 8442, 1

Sugerman, B. E. K., Ercolano, B., Barlow, M. J., et al. 2006, Science, 313, 196

Szalai, T., \& Vinkó, J. 2013, A\&A, 549, A79

Szalai, T., Vinkó, J., Balog, Z., et al. 2011, A\&A, 527, A61

Szalai, T., Vinkó, J., Nagy, A. P., et al. 2016, MNRAS, 460, 1500

Szczygiel, D. M., Kochanek, C. S., Dai, X. 2012, ApJ, 760, 20

Taubenberger, S., Pastorello, A., Mazzali, P. A., et al. 2006, MNRAS, 371, 1459

Thrasher, P., Li, W., \& Filippenko, A. V. 2008, CBET, 1507, 1

Tinyanont, S., Kasliwal, M. M., Fox, O. D., et al. 2016, ApJ, 833, 231

Tomasella, L.; Pastorello, A.; Valenti, S.; Benetti, S.

Xue, M., Jiang, B. W., Gao, J., Liu, J., Wang, S., Li, A. 2016, ApJS, 224, 23

Yamanaka, M., Maeda, K., Tanaka, M., et al. 2016, PASJ, 68, 68

Valenti, S., Pastorello, A., Cappellaro, E., et al. 2012, ATel, 4076, 1

Van Dyk, S. 2013, AJ, 145, 118

Vinkó, J., Pooley, D., Silverman, J. M., et al. 2017, ApJ, 837, 62

Wenger, M., Ochsenbein, F., Egret, D., et al. 2000, A\&AS, 143, 9 
Wesson, R., Barlow, M. J., Matsuura, M., \& Ercolano, B. 2015, MNRAS, 446, 2089

Williams, B. J., \& Fox, O. D. 2015, ApJ, 808, L22
Zheng, W., Li, W., Filippenko, A. V., \& Cenko, S. B. 2014, ATel, 5770, 1 


\section{APPENDIX}

\section{A. BASIC DATA AND MID-IR PHOTOMETRY OF THE STUDIED SNE}

Table A1. Basic parameters of the studied SNe with positive Spitzer detection

\begin{tabular}{|c|c|c|c|c|c|c|c|c|}
\hline Object & Type & $\begin{array}{c}\text { Discovery } \\
\text { (MJD) }\end{array}$ & Host & RA (J2000) & Dec (J2000) & $\begin{array}{c}d \\
(\mathrm{Mpc})\end{array}$ & $\mathrm{E}(\mathrm{B}-\mathrm{V})$ & $\begin{array}{l}\text { Refe- } \\
\text { rences }\end{array}$ \\
\hline SN 1993J & $\mathrm{IIb}$ & 49074 & NGC 3031 & $09: 55: 24.77$ & $+69: 01: 13.7$ & $3.6 \pm 0.2$ & 0.07 & 1 \\
\hline SN 1995N & IIn & 49842 & MCG-02-38-17 & $14: 49: 28.29$ & $-10: 10: 14.0$ & $24.0 \pm 4.0$ & 0.10 & 2 \\
\hline${ }^{*} \mathrm{SN} 2001 \mathrm{em}$ & $\mathrm{Ib} / \mathrm{c}$ & 52172 & UGC 11794 & $21: 42: 23.60$ & $+12: 29: 50.3$ & $71.6 \pm 0.7$ & 0.10 & 3,4 \\
\hline SN 2001gd & $\mathrm{IIb}$ & 52237 & NGC 5033 & 13:13:23.89 & $+36: 38: 17.7$ & $16.5 \pm 7.0$ & 0.01 & 5 \\
\hline SN 2002bu & IIn & 52361 & NGC 4242 & $12: 17: 37.18$ & $+45: 38: 47.4$ & $5.8 \pm 1.5$ & 0.01 & 6 \\
\hline SN 2002ed & II-P & 52482 & NGC 5468 & 14:06:38.22 & $-05: 27: 28.0$ & $43.8 \pm 7.0$ & 0.02 & 7,8 \\
\hline SN 2002hh & II-P & 52577 & NGC 6946 & $20: 34: 44.29$ & $+60: 07: 19.0$ & $5.9 \pm 0.4$ & 0.30 & 9 \\
\hline SN 2002ic & Ia-CSM & 52591 & $\mathrm{~A} 013002+2153$ & 01:30:02.55 & $+21: 53: 06.9$ & $280.0^{\dagger}$ & 0.05 & 10 \\
\hline SN 2003gd & II-P & 52802 & NGC 628 & $01: 36: 42.65$ & $+15: 44: 19.9$ & $8.9 \pm 3.2$ & 0.06 & 11 \\
\hline SN 2003lo & IIn & 53004 & NGC 1376 & 03:37:05.12 & $-05: 02: 17.3$ & $41.2 \pm 24.0$ & 0.04 & 12 \\
\hline SN 2004A & II-P & 53011 & NGC 6207 & $16: 43: 01.90$ & $+36: 50: 12.5$ & $20.3 \pm 2.1$ & 0.06 & 13 \\
\hline${ }^{*} \mathrm{SN} 2004 \mathrm{G}$ & II & 53023 & NGC 5668 & $14: 33: 21.40$ & $+04: 26: 49.5$ & $26.9 \pm 6.9$ & 0.12 & 14 \\
\hline SN $2004 \mathrm{~W}$ & Ia & 53032 & NGC 4649 & $12: 43: 36.52$ & $+11: 31: 50.8$ & $17.8 \pm 0.2$ & 0.02 & 15 \\
\hline SN 2004bv & Ia & 53149 & NGC 6907 & $20: 25: 06.34$ & $-24: 48: 53.7$ & $32.4 \pm 6.2$ & 0.06 & 16,17 \\
\hline SN 2004dj & II-P & 53187 & NGC 2403 & 07:37:17.02 & $+65: 35: 57.8$ & $3.5 \pm 0.4$ & 0.03 & 18 \\
\hline SN 2004et & II-P & 53270 & NGC 6946 & $20: 35: 25.33$ & $+60: 07: 17.7$ & $5.9 \pm 0.4$ & 0.30 & 19 \\
\hline SN 2005A & Ia & 53375 & NGC 958 & $02: 30: 43.25$ & $-02: 56: 19.8$ & $65.2 \pm 10.0$ & 0.03 & 20,21 \\
\hline SN 2005P & $\operatorname{Iax}$ & 53391 & NGC 5468 & 14:06:34.01 & $-05: 27: 42.6$ & $43.8 \pm 7.0$ & 0.03 & 22,23 \\
\hline SN $2005 \mathrm{~W}$ & Ia & 53402 & NGC 691 & 01:50:45.75 & $+21: 45: 35.6$ & $36.2 \pm 4.3$ & 0.07 & 24,25 \\
\hline SN 2005ad & II-P & 53407 & NGC 941 & $02: 28: 29.40$ & $-01: 08: 20.0$ & $20.8 \pm 2.2$ & 0.04 & 13 \\
\hline SN 2005af & II-P & 53379 & NGC 4945 & $13: 04: 44.10$ & $-49: 33: 59.8$ & $3.9 \pm 0.3$ & 0.18 & 13 \\
\hline SN 2005at & Ic & 53434 & NGC 6744 & 19:09:53.57 & $-63: 49: 22.8$ & $9.5 \pm 0.6$ & 0.04 & 26 \\
\hline SN 2005cp & IIn & 53542 & UGC 12886 & $23: 59: 30.88$ & $+18: 12: 09.6$ & $127.0 \pm 8.8$ & 0.03 & 27 \\
\hline SN 2005df & Ia & 53586 & NGC 1559 & $04: 17: 37.85$ & $-62: 46: 09.5$ & $14.9 \pm 3.6$ & 0.03 & 28 \\
\hline SN 2005gj & Ia-CSM & 53589 & A030111-0033 & 03:01:11.96 & $-00: 33: 13.9$ & $268.0^{\dagger}$ & 0.10 & 10 \\
\hline SN 2005gn & IIn & 53586 & ESO 488-G30 & 05:48:49.07 & $-24: 22: 45.5$ & $165.0^{\dagger}$ & 0.03 & 27 \\
\hline SN 2005ip & IIn & 53679 & NGC 2906 & 09:32:06.42 & $+08: 26: 44.4$ & $30.0 \pm 7.2$ & 0.04 & 27 \\
\hline SN 2006E & $\mathrm{Ia}$ & 53747 & NGC 5338 & $03: 53: 28.65$ & $+05: 12: 22.8$ & $12.8^{\dagger}$ & 0.03 & 29,30 \\
\hline SN 2006X & Ia & 53770 & NGC 4321 & $12: 22: 53.93$ & $+15: 48: 32.0$ & $3.6 \pm 0.2$ & 0.02 & 31 \\
\hline SN 2006bc & II-P & 53819 & NGC 2397 & $07: 21: 16.50$ & $-68: 59: 57.3$ & $14.7 \pm 2.0$ & 0.18 & 13 \\
\hline SN 2006bp & II-P & 53834 & NGC 3953 & $11: 53: 55.74$ & $+52: 21: 09.4$ & $17.5 \pm 2.0$ & 0.03 & 13 \\
\hline SN 2006ce & Ia & 53865 & NGC 908 & $02: 22: 54.63$ & $-21: 14: 29.4$ & $15.8 \pm 2.7$ & 0.02 & 32,33 \\
\hline SN 2006jc & Ibn & 54017 & UGC 4904 & 09:17:20.78 & $+41: 54: 32.7$ & $23.1 \pm 5.0$ & 0.02 & 34 \\
\hline SN 2006jd & IIn & 54020 & UGC 4179 & 08:02:07.43 & $+00: 48: 31.5$ & $77.0 \pm 5.0$ & 0.05 & 27 \\
\hline SN 2006mq & Ia & 54030 & ESO 494-G26 & 08:06:12.39 & $-27: 33: 45.4$ & $12.5 \pm 0.6$ & 0.36 & 35 \\
\hline SN 2006my & II-P & 53953 & NGC 4651 & $12: 43: 40.70$ & $+16: 23: 14.1$ & $22.3 \pm 2.2$ & 0.03 & 13 \\
\hline SN 2006ov & II-P & 53964 & NGC 4303 & 12:21:55.30 & $+04: 29: 16.7$ & $12.6 \pm 1.2$ & 0.02 & 13 \\
\hline
\end{tabular}

Table A1 continued on next page 
Spitzer SNE

Table A1 (continued)

\begin{tabular}{|c|c|c|c|c|c|c|c|c|}
\hline Object & Type & $\begin{array}{c}\text { Discovery } \\
\text { (MJD) }\end{array}$ & Host & RA (J2000) & Dec (J2000) & $\begin{array}{c}d \\
(\mathrm{Mpc})\end{array}$ & $\mathrm{E}(\mathrm{B}-\mathrm{V})$ & $\begin{array}{l}\text { Refe- } \\
\text { rences }\end{array}$ \\
\hline SN 2006qq & IIn & 54069 & ESO 553-G36 & 05:19:50.30 & $-20: 58: 06.4$ & $119.0 \pm 5.0$ & 0.02 & 27 \\
\hline SN 2007af & Ia & 54160 & NGC 5584 & $14: 22: 21.03$ & $-00: 23: 37.6$ & $22.1 \pm 5.6$ & 0.03 & 31 \\
\hline SN 2007gr & Ic & 54327 & NGC 1058 & $02: 43: 27.98$ & $+37: 20: 44.7$ & $9.3 \pm 1.2$ & 0.05 & 36 \\
\hline SN 2007it & II-P & 54356 & NGC 5530 & $14: 18: 25.63$ & $-43: 22: 53.8$ & $11.9 \pm 1.5$ & 0.10 & 37 \\
\hline SN 2007le & Ia & 54386 & NGC 7721 & $23: 38: 48.45$ & $-06: 31: 21.7$ & $22.2 \pm 3.2$ & 0.03 & 31 \\
\hline SN 2007oc & II-P & 54408 & NGC $7418 \mathrm{~A}$ & $22: 56: 41.8$ & $-36: 46: 22.3$ & $28.0 \pm 3.2$ & 0.02 & 13 \\
\hline SN 2007od & II-P & 54406 & UGC 12846 & $23: 55: 48.68$ & $+18: 24: 54.8$ & $26.7 \pm 3.2$ & 0.03 & 38 \\
\hline SN 2007rt & IIn & 54423 & UGC 6109 & 11:02:34.29 & $+50: 34: 58.5$ & $93.0 \pm 8.0$ & 0.01 & 27 \\
\hline SN 2007sq & II-P & 54442 & MCG-03-23-05 & $08: 47: 16.13$ & $-20: 01: 27.6$ & $72.7 \pm 10.7$ & 0.18 & 39 \\
\hline SN 2007sr & Ia & 54452 & NGC 4038 & $12: 01: 52.80$ & $-18: 58: 21.7$ & $21.5 \pm 4.2$ & 0.04 & 31 \\
\hline SN 2008J & IIn & 54480 & MCG -02-07-33 & $02: 34: 24.20$ & $-10: 50: 38.5$ & $66.0 \pm 5.0$ & 0.02 & 27 \\
\hline SN 2008Q & Ia & 54491 & NGC 524 & $01: 24: 57.23$ & $+09: 33: 01.5$ & $26.4 \pm 4.2$ & 0.07 & 31 \\
\hline SN 2008cg & IIn & 54591 & PGC 91487 & $15: 54: 15.15$ & $+10: 58: 25.0$ & $122.0^{\dagger}$ & 0.05 & 27 \\
\hline SN 2008en & IIn & 54681 & UGC 564 & $00: 55: 13.56$ & $+35: 26: 26.2$ & $151.0 \pm 5.0$ & 0.04 & 27 \\
\hline SN 2008fq & II & 54724 & NGC 6907 & $20: 25: 06.19$ & $-24: 48: 27.6$ & $32.4 \pm 6.2$ & 0.06 & 40,41 \\
\hline SN 2008gm & IIn & 54761 & NGC 7530 & $23: 14: 12.39$ & $-02: 46: 52.4$ & $48.0 \pm 3.5$ & 0.04 & 27 \\
\hline SN 2008ip & IIn & 54650 & NGC 4846 & $12: 57: 50.20$ & $+36: 22: 33.5$ & $63.0 \pm 5.0$ & 0.01 & 27 \\
\hline SN 2008jb & II & 54789 & ESO $302-G 13$ & 03:51:44.66 & $-38: 27: 00.1$ & $9.6 \pm 3.0$ & 0.01 & 42 \\
\hline SN 2009E & II-P & 54834 & NGC 4141 & $12: 09: 49.56$ & $+58: 50: 50.3$ & $37.5^{\dagger}$ & 0.02 & 43,44 \\
\hline SN $2009 \mathrm{H}$ & II & 54833 & NGC 1084 & $02: 45: 58.36$ & $-07: 35: 00.3$ & $17.5 \pm 1.0$ & 0.03 & 45,46 \\
\hline SN 2009af & II & 54878 & UGC 1551 & 02:03:36.37 & $+24: 04: 40.9$ & $35.6^{\dagger}$ & 0.08 & 47,48 \\
\hline SN 2009at & II & 54901 & NGC 5301 & $13: 46: 26.68$ & $+46: 06: 09.1$ & $23.0 \pm 2.3$ & 0.02 & 49,50 \\
\hline SN 2009em & Ic & 54956 & NGC 157 & $00: 34: 44.53$ & $-08: 23: 57.6$ & $16.8 \pm 5.0$ & 0.04 & 51,52 \\
\hline SN 2009gj & $\mathrm{IIb}$ & 55002 & NGC 134 & 00:30:28.56 & $-33: 12: 56.0$ & $17.1 \pm 3.2$ & 0.02 & 53,54 \\
\hline SN 2009ig & Ia & 55063 & NGC 1015 & $02: 38: 11.62$ & $-01: 18: 45.3$ & $36.6 \pm 1.5$ & 0.03 & 31 \\
\hline SN 2009ip & IIn & 56193 & NGC 7259 & $22: 23: 08.3$ & $-28: 56: 52$ & $20.5 \pm 2.0$ & 0.02 & 27 \\
\hline${ }^{*}$ SN 2009iu & $\mathrm{Ib}$ & 55075 & NGC 7329 & $22: 40: 10.35$ & $-66: 28: 06.4$ & $44.3 \pm 5.7$ & 0.03 & 55,56 \\
\hline SN 2009jf & $\mathrm{Ib}$ & 55101 & NGC 7479 & $23: 04: 52.98$ & $+12: 19: 59.5$ & $29.3 \pm 6.1$ & 0.10 & 57,58 \\
\hline SN 2009jr & Ia & 55112 & IC 1320 & $20: 26: 26.03$ & $+02: 54: 32.1$ & $70.0 \pm 5.0^{\dagger}$ & 0.12 & 59,60 \\
\hline SN 2009js & II-P p. & 55115 & NGC 918 & $02: 25: 48.28$ & $+18: 29: 25.8$ & $17.9 \pm 6.2$ & 0.31 & 61 \\
\hline SN 2009mk & $\mathrm{IIb}$ & 55180 & ESO 293-34 & 00:06:21.37 & $-41: 28: 59.8$ & $20.0 \pm 1.5$ & 0.02 & 62,63 \\
\hline SN 2010B & Ia & 55203 & NGC 5370 & $13: 54: 08.74$ & $+60: 40: 50.4$ & $43.0 \pm 3.2$ & 0.02 & 64,65 \\
\hline SN 2010F & II & 55209 & NGC 3120 & 10:05:21.05 & $-34: 13: 21.0$ & $32.2 \pm 7.6$ & 0.09 & 66,67 \\
\hline SN 2010gp & Ia & 55391 & NGC 6240 & $16: 52: 57.39$ & $+02: 23: 16.4$ & $100.0 \pm 7.5^{\dagger}$ & 0.07 & 68,69 \\
\hline SN 2010jl & IIn & 55503 & UGC 5189A & $09: 42: 53.33$ & $+09: 29: 41.8$ & $48.9 \pm 3.4$ & 0.02 & 70 \\
\hline SN 2010mc & IIn & 55428 & $\mathrm{~A} 172130+4807$ & $17: 21: 30.68$ & $+48: 07: 47.4$ & $159.0^{\dagger}$ & 0.02 & 71,72 \\
\hline SN 2011A & IIn & 55563 & NGC 4902 & 13:01:01.19 & $-14: 31: 34.8$ & $33.8 \pm 3.5$ & 0.04 & 73,74 \\
\hline SN 2011ae & Ia & 55604 & MCG-03-30-19 & $11: 54: 49.25$ & $-16: 51: 43.6$ & $27.5^{\dagger}$ & 0.05 & 75,76 \\
\hline SN 2011dh & $\mathrm{IIb}$ & 55713 & NGC 5194 & 13:30:05.11 & $+47: 10: 10.9$ & $7.7 \pm 0.5$ & 0.02 & 77 \\
\hline SN 2011dq & II & 55696 & NGC 337 & $00: 59: 47.75$ & $-07: 34: 20.5$ & $20.7 \pm 2.6$ & 0.10 & 78 \\
\hline SN 2011dx & Ia-pec. & 55739 & NGC 1376 & 03:37:05.61 & $-05: 01: 56.3$ & $60.8 \pm 3.0$ & 0.04 & 79,80 \\
\hline SN 2011fe & Ia & 55816 & NGC 5457 & 14:03:05.71 & $+54: 16: 25.2$ & $6.1 \pm 0.5$ & 0.01 & 31 \\
\hline
\end{tabular}

Table A1 continued on next page 
SzALAI ET AL.

Table A1 (continued)

\begin{tabular}{|c|c|c|c|c|c|c|c|c|}
\hline Object & Type & $\begin{array}{c}\text { Discovery } \\
\text { (MJD) }\end{array}$ & Host & RA (J2000) & $\operatorname{Dec}(\mathrm{J} 2000)$ & $\begin{array}{c}d \\
(\mathrm{Mpc})\end{array}$ & $\mathrm{E}(\mathrm{B}-\mathrm{V})$ & $\begin{array}{l}\text { Refe- } \\
\text { rences }\end{array}$ \\
\hline SN 2011fh & IIn & 55797 & NGC 4806 & $12: 56: 14.01$ & $-29: 29: 54.82$ & $33.3 \pm 2.6^{\dagger}$ & 0.04 & 81,82 \\
\hline${ }^{*} \mathrm{SN} 2011 \mathrm{ft}$ & $\mathrm{Ib}$ & 55803 & UGC 11021 & $17: 52: 42.98$ & $+29: 04: 10.6$ & $101.0 \pm 3.0$ & 0.06 & 83,84 \\
\hline SN 2011ht & IIn & 55833 & UGC 5460 & 10:08:10.56 & $+51: 50: 57.12$ & $17.8 \pm 3.0$ & 0.01 & 85,86 \\
\hline${ }^{*} \mathrm{SN} 2011 \mathrm{ir}$ & IIn & 55886 & UGC 6771 & 11:48:00.32 & $+04: 29: 47.1$ & $70.3 \pm 11.6$ & 0.02 & 87 \\
\hline SN 2011iy & Ia & 55904 & NGC 4984 & 13:08:58.39 & $-15: 31: 04.1$ & $21.3 \pm 2.1$ & 0.06 & 88,89 \\
\hline SN 2011ja & II-P & 55906 & NGC 4945 & 13:05:11.12 & $-49: 31: 27.0$ & $3.8 \pm 0.5$ & 0.15 & 90 \\
\hline $\mathrm{PTF} 11 \mathrm{kx}$ & Ia-CSM & 55579 & anon. & $08: 09: 12.87$ & $+46: 18: 48.8$ & $200.0^{\dagger}$ & 0.04 & 91 \\
\hline PTF11qcj & Ic & 55840 & anon. & $13: 13: 41.51$ & $+47: 17: 57.0$ & $125.0^{\dagger}$ & 0.01 & 92 \\
\hline SN 2012aw & II-P & 56002 & NGC 3351 & $10: 43: 53.73$ & $+11: 40: 17.6$ & $8.8 \pm 1.1$ & 0.02 & 93 \\
\hline SN 2012ca & Ia-CSM & 56042 & ESO 336-09 & $18: 41: 07.25$ & $-41: 47: 38.4$ & $80.0 \pm 6.0^{\dagger}$ & 0.07 & 94,95 \\
\hline *SN 2012cd & $\mathrm{IIb}$ & 56053 & MCG+09-22-53 & $13: 22: 35.25$ & $+54: 48: 47.7$ & $50.0 \pm 3.7^{\dagger}$ & 0.03 & 96,97 \\
\hline SN 2012cg & Ia & 56082 & NGC 4424 & $12: 27: 12.83$ & $+09: 25: 13.2$ & $7.3 \pm 0.5$ & 0.02 & 31 \\
\hline SN 2012fh & Ic & 56218 & NGC 3344 & 10:43:34.05 & $+24: 53: 29.0$ & $9.8 \pm 2.5$ & 0.03 & 98 \\
\hline${ }^{*} \mathrm{SN} 2013 \mathrm{E}$ & Ia & 56296 & IC 2532 & 10:00:05.52 & $-34: 14: 01.3$ & $27.2 \pm 6.8$ & 0.09 & 99 \\
\hline SN 2013L & IIn & 56314 & ESO 216-G39 & $11: 45: 29.55$ & $-50: 35: 53.1$ & $75.0 \pm 5.0^{\dagger}$ & 0.11 & 35 \\
\hline SN 2013ai & II & 56352 & NGC 2207 & $06: 16: 18.35$ & $-21: 22: 32.9$ & $14.3 \pm 2.0$ & 0.08 & 35 \\
\hline SN 2013am & II & 56372 & NGC 3623 & 11:18:56.95 & $+13: 03: 49.4$ & $18.5 \pm 2.2$ & 0.02 & 35 \\
\hline SN 2013bu & II & 56403 & NGC 7331 & $22: 37: 02.17$ & $+34: 24: 05.2$ & $13.1 \pm 1.5$ & 0.08 & 35 \\
\hline SN 2013cj & IIn & 56421 & UGC 10685 & 17:04:52.95 & $+12: 55: 10.4$ & $135.0 \pm 10.0^{\dagger}$ & 0.09 & 100 \\
\hline SN 2013df & IIb & 56447 & NGC 4414 & $12: 26: 29.33$ & $+31: 13: 38.3$ & $16.6 \pm 0.4$ & 0.02 & 101 \\
\hline SN 2013dk & Ic & 56465 & NGC 4038 & $12: 01: 52.72$ & $-18: 52: 18.3$ & $21.5 \pm 4.2$ & 0.04 & 35 \\
\hline SN 2013dn & Ia-CSM & 56457 & PGC 71942 & $23: 37: 45.74$ & $+14: 42: 37.1$ & $230.0^{\dagger}$ & 0.07 & 102 \\
\hline SN 2013dy & Ia & 56483 & NGC 7250 & $22: 18: 17.60$ & $+40: 34: 09.6$ & $13.7 \pm 4.4$ & 0.13 & 103 \\
\hline SN 2013ee & II & 56486 & NGC 3079 & 10:01:56.83 & $+55: 41: 44.0$ & $14.9 \pm 4.0$ & 0.01 & 104 \\
\hline SN 2013ej & II-P/L & 56497 & NGC 628 & $01: 36: 48.16$ & $+15: 45: 31.0$ & $9.5 \pm 0.6$ & 0.06 & 105 \\
\hline SN 2013ff & Ic & 56535 & NGC 2748 & 09:13:38.88 & $+76: 28: 10.8$ & $20.2 \pm 2.8$ & 0.03 & 106 \\
\hline SN 2014C & $\mathrm{Ib}$ & 56662 & NGC 7331 & $22: 37: 05.60$ & $+34: 24: 31.9$ & $13.1 \pm 1.0$ & 0.08 & 35 \\
\hline SN 2014G & II-L & 56671 & NGC 3448 & $10: 54: 34.13$ & $+54: 17: 56.9$ & $24.5^{\dagger}$ & 0.01 & 107,108 \\
\hline SN 2014J & Ia & 56677 & NGC 3034 & $09: 55: 42.12$ & $+69: 40: 25.9$ & $3.3 \pm 0.2$ & 0.14 & 31 \\
\hline SN 2014L & Ic & 56683 & NGC 4254 & $12: 18: 48.68$ & $+14: 24: 43.5$ & $16.8 \pm 1.2$ & 0.03 & 35 \\
\hline SN 2014bi & II-P & 56809 & NGC 4096 & 12:06:02.99 & $+47: 29: 33.5$ & $11.6 \pm 2.5$ & 0.02 & 35 \\
\hline SN 2014cx & II-P & 56902 & NGC 337 & $00: 59: 47.83$ & $-07: 34: 18.6$ & $19.6 \pm 2.6$ & 0.11 & 109,110 \\
\hline SN 2014df & $\mathrm{Ib}$ & 56811 & NGC 1448 & $03: 44: 23.99$ & $-44: 40: 08.1$ & $13.4 \pm 2.0$ & 0.01 & 35 \\
\hline SN 2014dt & $\operatorname{Iax}$ & 56950 & NGC 4303 & $12: 21: 57.57$ & $+04: 28: 18.5$ & $18.7 \pm 7.3$ & 0.02 & 111 \\
\hline
\end{tabular}

Table A1 continued on next page 
Spitzer SNE

Table A1 (continued)

\begin{tabular}{ccccccccc}
\hline \hline Object & Type & Discovery & Host & RA (J2000) & Dec (J2000) & $d$ & E(B-V) & Refe- \\
& & & & & rences \\
\hline
\end{tabular}

Note- ${ }^{*}$ Positive detection based on single-epoch imaging, see text for details. References: ${ }^{1}$ Filippenko et al. (1993); ${ }^{2}$ Van Dyk (2013); ${ }^{3}$ Papenkova et al. (2001); ${ }^{4}$ Bietenholz \& Bartel (2005); ${ }^{5}$ Matheson et al. (2001); ${ }^{6}$ Szczygiel et al. (2012); ${ }^{7}$ Monard \& Li (2002); ${ }^{8}$ Salvo \& Price (2002); ${ }^{9}$ Meikle et al. (2006); ${ }^{10}$ Fox \& Filippenko (2013); ${ }^{11}$ Sugerman et al. (2006); ${ }^{12}$ Meikle et al. (2005); ${ }^{13}$ Szalai \& Vinkó (2013); ${ }^{14}$ Nakano et al. (2004a); ${ }^{15}$ Moore et al. (2004); ${ }^{16}$ Nakano et al. (2004b); ${ }^{17} \mathrm{Li}$ et al. (2004); ${ }^{18}$ Szalai et al. (2011); ${ }^{19}$ Kotak et al. (2009); ${ }^{20}$ Graham \& Li (2005); ${ }^{21}$ Modjaz et al. (2005); ${ }^{22}$ Burket \& Li (2005); ${ }^{23}$ Jha et al. (2006); ${ }^{24}$ Nakano et al. (2005); ${ }^{25}$ Elias-Rosa et al. (2005); ${ }^{26}$ Kankare et al. (2014); ${ }^{27}$ Fox et al. (2011); ${ }^{28}$ Gerardy et al. (2007); ${ }^{29}$ Puckett et al. (2006); ${ }^{30}$ ?; ${ }^{31}$ Johansson et al. (2017); ${ }^{32}$ Ponticello et al. (2006); ${ }^{33}$ Blackman et al. (2006); ${ }^{34}$ Mattila et al. (2008); ${ }^{35}$ Tinyanont et al. (2016); ${ }^{36}$ Kochanek et al. (2011); ${ }^{37}$ Andrews et al. (2011b); ${ }^{38}$ Andrews et al. (2010); ${ }^{39}$ Lennarz et al. (2012); ${ }^{40}$ Thrasher et al. (2008); ${ }^{41}$ Quinn et al. (2008); ${ }^{42}$ Prieto et al. (2012); ${ }^{43}$ Boles (2009); ${ }^{44}$ Navasardyan et al. (2009); ${ }^{45} \mathrm{Li}$ et al. (2009a); ${ }^{46}$ Benetti et al. (2009); ${ }^{47}$ Cortini (2009); ${ }^{48}$ Ciroi et al. (2009); ${ }^{49}$ Nakano et al. (2009); ${ }^{50}$ Harutyunyan et al. (2009); ${ }^{51}$ Monard (2009); ${ }^{52}$ Navasardyan \& Benetti (2009); ${ }^{53}$ Marples et al. (2009); ${ }^{54}$ Foley (2009); ${ }^{55}$ Maza et al. (2009); ${ }^{56}$ Morrell \& Phillips (2009); ${ }^{57} \mathrm{Li}$ et al. (2009b); ${ }^{58}$ Kasliwal et al. (2009); ${ }^{59}$ Arbour \& Briggs (2009); ${ }^{60}$ Silverman et al. (2009); ${ }^{61}$ Gandhi et al. (2013); ${ }^{62}$ Marples \& Drescher (2009); ${ }^{63}$ Chornock \& Berger (2009); ${ }^{64}$ Nakano et al. (2010); ${ }^{65}$ Kasliwal (2010); ${ }^{66}$ Maza et al. (2010a); ${ }^{67}$ Foley (2010); ${ }^{68}$ Maza et al. (2010b); ${ }^{69}$ Folatelli et al. (2010); ${ }^{70}$ Fox et al. (2013); ${ }^{71}$ Howell \& Murray (2010); ${ }^{72}$ Ofek et al. (2013); ${ }^{73}$ Pignata et al. (2011); ${ }^{74}$ Stritzinger et al. (2011); ${ }^{75}$ Howerton et al. (2011); ${ }^{76} \mathrm{Sahu}$ et al. (2011a); ${ }^{77} \mathrm{Helou}$ et al. (2013); ${ }^{78}$ Monard et al. (2011b); ${ }^{79}$ Monard \& Prieto (2011); ${ }^{80}$ Prieto (2011a); ${ }^{81}$ Monard et al. (2011a); ${ }^{82}$ Prieto \& Seth (2011); ${ }^{83}$ Balanutsa et al. (2011); ${ }^{84}$ Prieto (2011b); ${ }^{85}$ Boles (2011); ${ }^{86}$ Prieto et al. (2011); ${ }^{87}$ Tomasella et al. (2011); ${ }^{88}$ Itagaki et al. (2011); ${ }^{89}$ Chen et al. (2011); ${ }^{90}$ Andrews et al. (2016); ${ }^{91}$ Graham et al. (2017); ${ }^{92}$ Corsi et al. (2014); ${ }^{93}$ Siviero et al. (2012); ${ }^{94}$ Drescher et al. (2012); ${ }^{95}$ Valenti et al. (2012); ${ }^{96}$ Shurpakov et al. (2012); ${ }^{97}$ Marion et al. (2012); ${ }^{98}$ Nakano et al. (2012); ${ }^{99}$ Morrell et al. (2006); ${ }^{100}$ Jin et al. (2013); ${ }^{101}$ Szalai et al. (2016); ${ }^{102}$ Drake et al. (2013); ${ }^{103}$ Casper et al. (2013); ${ }^{104}$ Cortini (2013); ${ }^{105}$ Mauerhan et al. (2017); ${ }^{106}$ Brimacombe et al. (2013); ${ }^{107}$ Ochner et al. (2014); ${ }^{108}$ Zheng et al. (2014); ${ }^{109}$ Holoien et al. (2014); ${ }^{110}$ Andrews et al. (2015); ${ }^{111}$ Fox et al. (2016). Additional notes: ${ }^{\dagger}$ Distance calculated from redshift. 
Table A2. Mid-IR fluxes of SNe with previously unreported Spitzer photometry

\begin{tabular}{|c|c|c|c|c|c|c|c|c|}
\hline Object & Type & $\begin{array}{l}\text { MJD - } \\
2450000\end{array}$ & $\begin{array}{l}\text { Epoch }^{\dagger} \\
\text { (days) }\end{array}$ & $\begin{array}{c}F_{\nu,[3.6]} \\
(\mu \mathrm{Jy})\end{array}$ & $\begin{array}{c}F_{\nu,[4.5]} \\
(\mu \mathrm{Jy})\end{array}$ & $\begin{array}{c}F_{\nu,[5.8]} \\
(\mu \mathrm{Jy})\end{array}$ & $\begin{array}{c}F_{\nu,[8.0]} \\
(\mu \mathrm{Jy})\end{array}$ & $\begin{array}{l}\text { SN-targeted } \\
\text { observation? }\end{array}$ \\
\hline${ }^{*} S N 2001 \mathrm{em}^{a}$ & $I b / c$ & 5330re & 1135 & $303(41)$ & $349(36)$ & $481(50)$ & $859(105)$ & Yes \\
\hline $\mathrm{SN} 2001 \mathrm{gd}^{b}$ & $\mathrm{IIb}$ & $53189^{f}$ & 952 & $11(8)$ & $12(7)$ & non ${ }^{\ddagger}$ & non $^{\ddagger}$ & No \\
\hline $\mathrm{SN} 2002 \mathrm{ed}^{c}$ & II-P & $53569^{g}$ & 1086 & non $\ddagger$ & $7(7)$ & non $\ddagger$ & non $^{\ddagger}$ & No \\
\hline${ }^{*} S N 2004 G^{a}$ & $I I$ & $53047^{h}$ & 24 & $249(28)$ & $395(33)$ & $265(30)$ & 196(29) & No \\
\hline $\mathrm{SN} 2004 \mathrm{~W}^{a, b}$ & Ia & $53166^{h}$ & 134 & non $^{\ddagger}$ & non $^{\ddagger}$ & non $^{\ddagger}$ & $212(29)$ & No \\
\hline $\mathrm{SN} 2004 \mathrm{bv}^{b}$ & Ia & $53308^{i}$ & 159 & $22(17)$ & non $^{\ddagger}$ & non $^{\ddagger}$ & non $^{\ddagger}$ & No \\
\hline $\mathrm{SN} 2005 \mathrm{~A}^{a, b}$ & Ia & $53387^{i}$ & 12 & $154(112)$ & $64(64)$ & non $\ddagger$ & non $^{\ddagger}$ & No \\
\hline $\mathrm{SN} 2005 \mathrm{P}^{b}$ & $\operatorname{Iax}$ & $53569^{g}$ & 178 & non $^{\ddagger}$ & non $^{\ddagger}$ & non $^{\ddagger}$ & $54(32)$ & Yes \\
\hline \multirow[t]{2}{*}{$\mathrm{SN} 2005 \mathrm{~W}^{a, b}$} & $\mathrm{Ia}$ & $53607^{g}$ & 205 & $8(8)$ & $5(5)$ & non $\ddagger$ & $177(28)$ & Yes \\
\hline & & $53770^{g}$ & 368 & non $^{\ddagger}$ & non $\ddagger$ & non $^{\ddagger}$ & $128(24)$ & Yes \\
\hline \multirow[t]{2}{*}{$\mathrm{SN} 2006 \mathrm{E}^{a, b}$} & Ia & $53955^{k}$ & 208 & $30(10)$ & $17(7)$ & $14(8)$ & $233(29)$ & Yes \\
\hline & & $54150^{k}$ & 403 & non $^{\ddagger}$ & non $^{\ddagger}$ & $21(8)$ & $54(14)$ & Yes \\
\hline${ }^{*} S N 2006 F^{a}$ & $I b$ & $53770^{l}$ & 24 & $400(43)$ & $301(34)$ & $n o n^{\ddagger}$ & $n o n^{\ddagger}$ & No \\
\hline \multirow[t]{2}{*}{$\mathrm{SN} 2006 \mathrm{ce}^{a, b}$} & Ia & $53955^{k}$ & 90 & $146(21)$ & $38(10)$ & $29(9)$ & $511(42)$ & No \\
\hline & & $54355^{m}$ & 490 & non ${ }^{\ddagger}$ & non $^{\ddagger}$ & non ${ }^{\ddagger}$ & $65(15)$ & No \\
\hline $\mathrm{SN} 2007 \mathrm{sq}^{b}$ & II-P & $54596^{n}$ & 154 & $224(35)$ & $\ldots$ & $330(45)$ & non $^{\ddagger}$ & No \\
\hline $\mathrm{SN} 2008 \mathrm{fq}^{c}$ & II & $55892^{\circ}$ & 1168 & $40(40)$ & $55(45)$ & $\ldots$ & $\ldots$ & No \\
\hline $\mathrm{SN} 2009 \mathrm{E}^{c}$ & II-P & $55381^{p}$ & 547 & $10(10)$ & $37(13)$ & $\ldots$ & $\ldots$ & No \\
\hline \multirow[t]{2}{*}{$S N 2009 H^{a}$} & $I I$ & $5507 r^{j}$ & 243 & 1312(104) & 1929(96) & $\ldots$ & $\ldots$ & No \\
\hline & & $55116^{j}$ & 282 & $1044(100)$ & $1672(88)$ & $\ldots$ & $\ldots$ & No \\
\hline \multirow[t]{2}{*}{$S N 2009 a f^{a}$} & $I I$ & $5507 r^{p}$ & 199 & 125(20) & 265(27) & $\ldots$ & $\ldots$ & No \\
\hline & & $55107 q$ & 229 & $79(15)$ & 188(23) & $\ldots$ & $\ldots$ & $N_{o}$ \\
\hline \multirow[t]{2}{*}{$S N 2009 a t^{a}$} & II & $55375^{r}$ & 474 & 161(34) & 172(28) & $\ldots$ & $\ldots$ & No \\
\hline & & $55406^{r}$ & 505 & $150(34)$ & 165(28) & $\ldots$ & $\ldots$ & No \\
\hline $\mathrm{SN} 2009 \mathrm{em}^{b}$ & Ic & $55073^{s}$ & 117 & $65(26)$ & $232(32)$ & $\ldots$ & $\ldots$ & No \\
\hline SN 2009gj ${ }^{a, b}$ & IIb & $55070^{s}$ & 68 & $244(26)$ & $450(35)$ & $\ldots$ & $\ldots$ & No \\
\hline${ }^{*} S N 2009 i u^{a}$ & $I b$ & $55142^{t}$ & 67 & $149(20)$ & $227(25)$ & $\ldots$ & $\ldots$ & No \\
\hline $\mathrm{SN} 2009 \mathrm{jf} \mathrm{f}^{c}$ & $\mathrm{Ib}$ & $55201^{u}$ & 100 & $180(25)$ & $307(31)$ & $\ldots$ & $\ldots$ & No \\
\hline \multirow[t]{3}{*}{$\mathrm{SN} 2009 \mathrm{jr}{ }^{b}$} & Ia & $55150^{v}$ & 38 & $54(54)$ & non $^{\ddagger}$ & $\ldots$ & $\ldots$ & Yes \\
\hline & & $55161^{v}$ & 49 & $45(45)$ & non $\ddagger$ & $\ldots$ & $\ldots$ & Yes \\
\hline & & $55171^{v}$ & 59 & $23(23)$ & non $^{\ddagger}$ & $\ldots$ & $\ldots$ & Yes \\
\hline $\mathrm{SN} 2009 \mathrm{mk}^{c}$ & $\mathrm{IIb}$ & $55405^{p}$ & 225 & $75(35)$ & $107(31)$ & $\ldots$ & $\ldots$ & No \\
\hline \multirow[t]{4}{*}{$S N 2010 B^{a}$} & $I a$ & $55246^{v}$ & 43 & 111(18) & $45(11)$ & $\ldots$ & $\ldots$ & Yes \\
\hline & & $55281^{v}$ & 78 & $73(15)$ & $31(9)$ & $\ldots$ & $\ldots$ & Yes \\
\hline & & $55311^{v}$ & 108 & $61(14)$ & $29(9)$ & $\ldots$ & $\ldots$ & Yes \\
\hline & & $55343^{i}$ & 140 & $59(13)$ & $33(10)$ & $\ldots$ & $\ldots$ & Yes \\
\hline \multirow[t]{3}{*}{$\mathrm{SN} 2010 \mathrm{~F}^{b}$} & II & $55416^{x}$ & 207 & $41(16)$ & $120(21)$ & $\ldots$ & $\ldots$ & Yes \\
\hline & & $55587^{x}$ & 378 & $10(10)$ & $25(12)$ & $\ldots$ & $\ldots$ & Yes \\
\hline & & $55740^{y}$ & 531 & non $^{\ddagger}$ & $5(5)$ & $\ldots$ & $\ldots$ & Yes \\
\hline $\mathrm{SN} 2010 \mathrm{gp}^{c}$ & $\mathrm{Ia}$ & $55498^{z}$ & 107 & $12(8)$ & $8(6)$ & $\ldots$ & $\ldots$ & No \\
\hline
\end{tabular}

Table A2 continued on next page 
Spitzer SNE

Table A2 (continued)

\begin{tabular}{|c|c|c|c|c|c|c|c|c|}
\hline Object & Type & $\begin{array}{c}\text { MJD - } \\
2450000\end{array}$ & $\begin{array}{l}\text { Epoch }^{\dagger} \\
\text { (days) }\end{array}$ & $\begin{array}{c}F_{\nu,[3.6]} \\
(\mu \mathrm{Jy})\end{array}$ & $\begin{array}{c}F_{\nu,[4.5]} \\
(\mu \mathrm{Jy})\end{array}$ & $\begin{array}{c}F_{\nu,[5.8]} \\
(\mu \mathrm{Jy})\end{array}$ & $\begin{array}{c}F_{\nu,[8.0]} \\
(\mu \mathrm{Jy})\end{array}$ & $\begin{array}{l}\text { SN-targeted } \\
\text { observation? }\end{array}$ \\
\hline \multirow[t]{2}{*}{$S N 2010 m c^{a}$} & IIn & $56814^{\varepsilon}$ & 1386 & $35(9)$ & $38(10)$ & $\ldots$ & $\ldots$ & Yes \\
\hline & & $57163^{w}$ & 1735 & $32(9)$ & $34(10)$ & $\ldots$ & $\ldots$ & Yes \\
\hline $\mathrm{SN} 2011 \mathrm{~A}^{a, c}$ & IIn & $55649^{\alpha}$ & 86 & $58(13)$ & $49(12)$ & $\ldots$ & $\ldots$ & No \\
\hline $\mathrm{SN} 2011 \mathrm{a} \mathrm{e}^{a, c}$ & Ia & $55616^{\beta}$ & 12 & $1037(55)$ & $724(46)$ & $\ldots$ & $\ldots$ & No \\
\hline SN $2011 \mathrm{dq}^{c}$ & II & $56955^{\gamma}$ & 1259 & $1230(61)$ & $963(55)$ & $\ldots$ & $\ldots$ & No \\
\hline SN $2011 d x^{c}$ & Ia-pec. & $55864^{\delta}$ & 125 & $7(6)$ & non $^{\ddagger}$ & $\ldots$ & $\ldots$ & No \\
\hline $\mathrm{SN} 2011 \mathrm{fh}^{a, c}$ & IIn & $56383^{\varepsilon}$ & 586 & $23(18)$ & $59(20)$ & $\cdots$ & $\cdots$ & Yes \\
\hline${ }^{*} S N 2011 f t^{a}$ & $I b$ & $56060^{j}$ & 257 & $81(15)$ & $\ldots$ & $\ldots$ & $\ldots$ & No \\
\hline $\mathrm{SN} 2011 \mathrm{ht}^{a, b}$ & IIn & $55833^{\zeta}$ & 638 & non $^{\ddagger}$ & $8(4)$ & $\ldots$ & $\ldots$ & Yes \\
\hline${ }^{*} S N 2011 i r^{a}$ & IIn & $55886^{\zeta}$ & 832 & $35(10)$ & $47(11)$ & $\ldots$ & $\ldots$ & Yes \\
\hline SN 2011iy ${ }^{c}$ & Ia & $56932^{\gamma}$ & 1028 & $\cdots$ & $5(5)$ & $\cdots$ & $\cdots$ & No \\
\hline \multirow[t]{3}{*}{$\mathrm{SN} 2012 \mathrm{aw}^{c, d}$} & II-P & $56360^{\varphi}$ & 358 & $135(14)$ & $521(35)$ & $\ldots$ & $\ldots$ & Yes \\
\hline & & $56489^{\varphi}$ & 487 & $38(10)$ & $166(20)$ & $\ldots$ & $\ldots$ & Yes \\
\hline & & $56700^{\iota}$ & 698 & $5(5)$ & $13(5)$ & $\ldots$ & $\ldots$ & Yes \\
\hline \multirow[t]{3}{*}{$S N$ 2012ca $a^{a, d}$} & $I a-C S M$ & $56446^{\zeta}$ & 404 & $812(86)$ & $963(74)$ & $\cdots$ & $\cdots$ & Yes \\
\hline & & $56619 \varsigma$ & 577 & $999(90)$ & 1362(80) & $\ldots$ & $\ldots$ & Yes \\
\hline & & $56820^{\zeta}$ & 778 & $328(77)$ & $716(67)$ & $\ldots$ & $\ldots$ & Yes \\
\hline${ }^{*} S N$ 2012cd ${ }^{a}$ & $I I b$ & $56090^{\eta}$ & 37 & $297(28)$ & $306(29)$ & $\ldots$ & $\ldots$ & No \\
\hline \multirow[t]{5}{*}{$\mathrm{SN} 2012 \mathrm{fh}^{a, c}$} & Ic & $56117^{\kappa}$ & 8 & $3324(96)$ & $2756(88)$ & $\cdots$ & $\cdots$ & No \\
\hline & & $56122^{\kappa}$ & 13 & $3640(102)$ & 2903(92) & $\cdots$ & $\cdots$ & No \\
\hline & & $56323^{\mu}$ & 214 & 1058(55) & 1916(74) & $\ldots$ & $\ldots$ & Yes \\
\hline & & $56356^{\mu}$ & 247 & $649(44)$ & $1292(61)$ & $\ldots$ & $\ldots$ & Yes \\
\hline & & $56692^{\iota}$ & 583 & non $^{\ddagger}$ & $16(5)$ & $\cdots$ & $\cdots$ & Yes \\
\hline${ }^{*} S N 2013 E^{a}$ & $I a$ & $56376^{j}$ & 80 & 111(18) & $\ldots$ & $\ldots$ & $\ldots$ & No \\
\hline \multirow[t]{2}{*}{$S N 2013 c j^{a}$} & IIn & $56815^{\varepsilon}$ & 394 & 998(52) & $916(51)$ & $\ldots$ & $\ldots$ & Yes \\
\hline & & $57168^{w}$ & 747 & $1097(56)$ & $1120(57)$ & $\cdots$ & $\cdots$ & Yes \\
\hline \multirow[t]{2}{*}{$S N 2013 d n^{a, d}$} & $I a-C S M$ & $56920^{\varepsilon}$ & 463 & $107(19)$ & 131(20) & $\ldots$ & $\ldots$ & Yes \\
\hline & & $57285^{w}$ & 828 & $38(11)$ & $47(12)$ & $\ldots$ & $\ldots$ & Yes \\
\hline \multirow[t]{2}{*}{ SN $2013 \mathrm{dy}^{b}$} & Ia & $56691^{\vartheta}$ & 208 & $34(19)$ & $7(7)$ & $\ldots$ & $\ldots$ & Yes \\
\hline & & $56731^{\iota}$ & 248 & $18(19)$ & $7(7)$ & $\ldots$ & $\ldots$ & Yes \\
\hline \multirow[t]{4}{*}{$\mathrm{SN} 2013 \mathrm{ee}^{a, b}$} & II & $56677^{\iota}$ & 191 & $120(40)$ & $417(35)$ & $\cdots$ & $\cdots$ & Yes \\
\hline & & $56706^{\iota}$ & 220 & $171(40)$ & $393(35)$ & $\ldots$ & $\ldots$ & Yes \\
\hline & & $56827^{\iota}$ & 341 & $106(40)$ & $178(35)$ & $\ldots$ & $\ldots$ & Yes \\
\hline & & $57057^{\nu}$ & 571 & $9(5)$ & $13(5)$ & $\ldots$ & $\ldots$ & Yes \\
\hline SN 2013ff ${ }^{c}$ & Ic & $56682^{\gamma}$ & 147 & 263(66) & $632(59)$ & $\ldots$ & $\ldots$ & No \\
\hline $\mathrm{SN} 2014 \mathrm{G}^{c}$ & II-L & $57232^{w}$ & 561 & $41(15)$ & $85(17)$ & $\ldots$ & $\ldots$ & Yes \\
\hline SN $2014 \mathrm{cx}^{c}$ & II-P & $56955^{\gamma}$ & 53 & $1312(60)$ & $992(53)$ & $\ldots$ & $\ldots$ & No \\
\hline
\end{tabular}

Table A2 continued on next page 
Table A2 (continued)

\begin{tabular}{|c|c|c|c|c|c|c|c|c|}
\hline Object & Type & $\begin{array}{l}\text { MJD - } \\
2450000\end{array}$ & $\begin{array}{l}\text { Epoch }^{\dagger} \\
\text { (days) }\end{array}$ & $\begin{array}{c}F_{\nu,[3.6]} \\
(\mu \mathrm{Jy})\end{array}$ & $\begin{array}{c}F_{\nu,[4.5]} \\
(\mu \mathrm{Jy})\end{array}$ & $\begin{array}{c}F_{\nu,[5.8]} \\
(\mu \mathrm{Jy})\end{array}$ & $\begin{array}{c}F_{\nu,[8.0]} \\
(\mu \mathrm{Jy})\end{array}$ & $\begin{array}{l}\text { SN-targeted } \\
\text { observation? }\end{array}$ \\
\hline
\end{tabular}

Note-Photometric methods: ${ }^{a}$ aperture photometry on the original images; ${ }^{b}$ ap. photometry after subtracting a very late-time image + flux differences; ${ }^{c}$ ap. photometry after subtracting a pre-explosion image + flux differences; $d$ "Fox+11 method" (see details in text). *Positive detection based on single-epoch imaging. Data marked with italic denote cases where image subtraction can not be applied (in all these cases, measured fluxes can be handled only as upper limits). Program IDs: ${ }^{e} 3641$ (PI van Dyk); ${ }^{f} 159$ (PI Kennicutt); ${ }^{g} 20256$ (PI Meikle); ${ }^{h} 69$ (PI Fazio); ${ }^{i} 3672$ (PI Mazzarella); ${ }^{j} 80072$ (PI Tully); ${ }^{k} 30292$ (PI Meikle); ${ }^{m} 40619$ (PI Kotak); ${ }^{n} 50111$ (PI McGaugh); ${ }^{o} 80089$ (PI Sanders); ${ }^{p} 61060$ (PI Sheth); ${ }^{q} 30496$ (PI Fisher); ${ }^{r} 80254$ (PI Stern); ${ }^{s} 61065$ (PI Sheth); ${ }^{t} 61003$ (PI Freedman); ${ }^{u} 61009$ (PI Freedman); ${ }^{v} 60166$ (PI Garnavich); ${ }^{w} 11053$ (PI Fox); ${ }^{x} 70008$ (PI Andrews); ${ }^{y} 80131$ (PI Andrews); ${ }^{z} 70038$ (PI Sanders); 10139 (PI Fox); ${ }^{\zeta} 90137$ (PI Fox); ${ }^{\alpha} 61066$ (PI Sheth); ${ }^{\beta} 61062$ (PI Sheth); ${ }^{\gamma} 10046$ (PI Sanders); ${ }^{\delta} 80023$ (PI Fox); ${ }^{\varphi} 90178$ (PI Andrews); ${ }^{\eta} 80069$ (PI Beygu); ${ }^{\vartheta} 10152$ (PI Kasliwal); ${ }^{\iota} 10136$ (PI Kasliwal); ${ }^{\kappa} 800025$ (PI van Zee); ${ }^{\mu} 80196$ (PI Kasliwal); ${ }^{\nu} 11063$ (PI Kasliwal). Additional notes: ${ }^{\dagger}$ days since discovery; ${ }^{\ddagger}$ no point source at the position of the SN. 
Table A3. 3.6 and $4.5 \mu \mathrm{m}$ absolute Vega magnitudes of all SNe positively detected by Spitzer/IRAC.

\begin{tabular}{|c|c|c|c|c|c|c|}
\hline \multirow[t]{2}{*}{ Object } & \multirow[t]{2}{*}{ Type } & \multirow{2}{*}{$\begin{array}{l}\text { MJD - } \\
2450000\end{array}$} & \multirow{2}{*}{$\begin{array}{l}\text { Epoch } \\
\text { (days) }\end{array}$} & \multicolumn{2}{|c|}{ Absolute mag. } & \multirow{2}{*}{$\begin{array}{c}\text { Source of } \\
\text { data }^{\dagger}\end{array}$} \\
\hline & & & & $3.6 \mu \mathrm{m}$ & $4.5 \mu \mathrm{m}$ & \\
\hline \multirow[t]{12}{*}{ SN 1993J } & \multirow[t]{12}{*}{ IIb } & 52948 & 3874 & $-11.67(0.10)$ & $-12.79(0.10)$ & 1 \\
\hline & & 52976 & 3902 & $-11.85(0.15)$ & $-12.93(0.10)$ & 1 \\
\hline & & 53125 & 4051 & $-11.48(0.10)$ & $-12.55(0.10)$ & 1 \\
\hline & & 53357 & 4283 & $-11.55(0.10)$ & $-12.62(0.10)$ & 1 \\
\hline & & 53497 & 4423 & $-11.71(0.10)$ & $-12.58(0.10)$ & 1 \\
\hline & & 53665 & 4591 & $-11.40(0.10)$ & $-12.47(0.10)$ & 1 \\
\hline & & 54419 & 5345 & $-11.15(0.10)$ & $-12.00(0.10)$ & 1 \\
\hline & & 55736 & 6662 & $-10.55(0.25)$ & $-10.93(0.25)$ & 1 \\
\hline & & 55936 & 6862 & $-10.88(0.25)$ & $-11.29(0.25)$ & 1 \\
\hline & & 56671 & 7597 & $-10.78(0.25)$ & $-11.15(0.25)$ & 1 \\
\hline & & 56699 & 7625 & $-10.45(0.25)$ & $-10.92(0.25)$ & 1 \\
\hline & & 56820 & 7746 & $-10.45(0.25)$ & $-10.71(0.25)$ & 1 \\
\hline$S N 1995 N$ & IIn & 54909 & 5067 & $-15.21(0.44)$ & $-16.51(0.39)$ & 2 \\
\hline$S N 2001 \mathrm{em}$ & $\mathrm{Ib} / \mathrm{c}$ & 53307 & 1135 & $-19.37(0.15)$ & $-20.00(0.11)$ & This work \\
\hline SN 2001gd & $\mathrm{IIb}$ & 53189 & 952 & $-12.59(1.00)$ & $-13.28(1.00)$ & This work \\
\hline SN 2002bu & IIn & 53120 & 759 & $-14.52(0.56)$ & $-15.47(0.56)$ & 3 \\
\hline SN 2002ed & $\mathrm{II}-\mathrm{P}$ & 53569 & 1086 & non & $-14.68(1.14)$ & This work \\
\hline \multirow[t]{3}{*}{$\mathrm{SN} 2002 \mathrm{hh}$} & \multirow[t]{3}{*}{$\mathrm{II}-\mathrm{P}$} & 53167 & 590 & non & $-14.13(0.51)$ & 4 \\
\hline & & 53261 & 684 & non & $-14.19(0.51)$ & 4 \\
\hline & & 53335 & 758 & non & $-13.12(0.98)$ & 4 \\
\hline \multirow[t]{3}{*}{ SN 2002ic } & \multirow[t]{3}{*}{$\mathrm{Ia}-\mathrm{CSM}$} & 53386 & 795 & $-20.63(0.18)$ & $-21.51(0.19)$ & 5 \\
\hline & & 53770 & 1179 & $-19.66(0.23)$ & $-20.66(0.20)$ & 5 \\
\hline & & 53961 & 1370 & $-19.26(0.24)$ & $-20.29(0.24)$ & 5 \\
\hline \multirow[t]{2}{*}{ SN 2003gd } & \multirow[t]{2}{*}{$\mathrm{II}-\mathrm{P}$} & 53211 & 409 & $-11.89(0.92)$ & $-13.74(0.81)$ & 6 \\
\hline & & 53385 & 583 & non & $-10.96(1.26)$ & 6 \\
\hline \multirow[t]{3}{*}{ SN 2003lo } & \multirow[t]{3}{*}{ IIn } & 53260 & 256 & $-17.63(0.34)$ & $-18.37(0.27)$ & 7 \\
\hline & & 53388 & 384 & $-16.76(0.58)$ & $-17.40(0.43)$ & 7 \\
\hline & & 53771 & 767 & non & $-15.49(1.09)$ & 7 \\
\hline \multirow[t]{3}{*}{ SN 2004A } & \multirow[t]{3}{*}{$\mathrm{II}-\mathrm{P}$} & 53258 & 247 & $-15.65(0.47)$ & $-16.50(0.46)$ & 8 \\
\hline & & 53456 & 445 & $-14.73(0.49)$ & $-15.05(0.49)$ & 8 \\
\hline & & 53574 & 563 & $-14.43(0.54)$ & $-14.67(0.56)$ & 8 \\
\hline$S N 2004 G$ & $I I$ & 53047 & 24 & $-17.03(0.57$ & $-18.01(0.56)$ & This work \\
\hline SN 2004bv & Ia & 53308 & 159 & $-14.80(0.48)$ & non & This work \\
\hline \multirow[t]{5}{*}{ SN 2004dj } & \multirow[t]{5}{*}{$\mathrm{II}-\mathrm{P}$} & 53285 & 98 & $-16.69(0.37)$ & $-17.02(0.37)$ & 9 \\
\hline & & 53286 & 99 & $-16.66(0.37)$ & $-17.03(0.37)$ & 9 \\
\hline & & 53290 & 103 & $-16.36(0.37)$ & $-17.00(0.37)$ & 9 \\
\hline & & 53310 & 123 & $-15.67(0.37)$ & $-16.75(0.37)$ & 9 \\
\hline & & 53454 & 267 & $-14.30(0.37)$ & $-15.89(0.37)$ & 9 \\
\hline
\end{tabular}

Table A3 continued on next page 
Szalai ET AL.

Table A3 (continued)

\begin{tabular}{|c|c|c|c|c|c|c|}
\hline \multirow[t]{2}{*}{ Object } & \multirow[t]{2}{*}{ Type } & \multirow{2}{*}{$\begin{array}{l}\text { MJD - } \\
2450000\end{array}$} & \multirow{2}{*}{$\begin{array}{l}\text { Epoch } \\
\text { (days) }\end{array}$} & \multicolumn{2}{|c|}{ Absolute mag. } & \multirow{2}{*}{$\begin{array}{c}\text { Source of } \\
\text { data }^{\dagger}\end{array}$} \\
\hline & & & & $3.6 \mu \mathrm{m}$ & $4.5 \mu \mathrm{m}$ & \\
\hline & & 53663 & 476 & $-14.74(0.37)$ & $-15.45(0.37)$ & 9 \\
\hline & & 53818 & 631 & $-14.39(0.37)$ & $-15.13(0.37)$ & 9 \\
\hline & & 54037 & 850 & $-13.88(0.37)$ & $-14.68(0.37)$ & 9 \\
\hline & & 54040 & 853 & $-13.87(0.37)$ & $-14.66(0.37)$ & 9 \\
\hline & & 54193 & 1006 & $-13.28(0.37)$ & $-14.07(0.37)$ & 9 \\
\hline & & 54424 & 1237 & $-12.93(0.37)$ & $-13.64(0.37)$ & 9 \\
\hline & & 54428 & 1241 & $-12.92(0.37)$ & $-13.63(0.37)$ & 9 \\
\hline & & 54429 & 1242 & $-12.94(0.37)$ & $-13.64(0.37)$ & 9 \\
\hline & & 54564 & 1377 & $-12.85(0.37)$ & $-13.45(0.37)$ & 9 \\
\hline & & 54569 & 1382 & $-12.84(0.37)$ & $-13.46(0.37)$ & 10 \\
\hline \multirow[t]{17}{*}{ SN 2004et } & $\mathrm{II}-\mathrm{P}$ & 53335 & 65 & $-18.38(0.15)$ & $-18.53(0.14)$ & 11 \\
\hline & & 53571 & 300 & $-14.93(0.15)$ & $-16.98(0.14)$ & 11 \\
\hline & & 53631 & 360 & $-14.36(0.14)$ & $-16.33(0.14)$ & 11 \\
\hline & & 53676 & 406 & $-14.02(0.15)$ & $-15.79(0.14)$ & 11 \\
\hline & & 53735 & 465 & $-13.37(0.16)$ & $-15.28(0.15)$ & 11 \\
\hline & & 53960 & 690 & non & $-12.39(0.38)$ & 11 \\
\hline & & 54006 & 736 & non & $-12.04(0.51)$ & 11 \\
\hline & & 54065 & 795 & non & $-11.59(0.20)$ & 11 \\
\hline & & 54098 & 828 & non & $-12.19(0.17)$ & 11 \\
\hline & & 54285 & 1015 & non & $-11.54(0.21)$ & 11 \\
\hline & & 54396 & 1125 & $-12.32(0.16)$ & $-13.94(0.15)$ & 11 \\
\hline & & 54461 & 1191 & $-12.09(0.17)$ & $-13.98(0.15)$ & 11 \\
\hline & & 54493 & 1222 & $-12.11(0.17)$ & $-14.09(0.15)$ & 11 \\
\hline & & 54665 & 1395 & $-12.47(0.34)$ & $-14.27(0.15)$ & 12 \\
\hline & & 55049 & 1779 & $-10.85(0.53)$ & $-13.37(0.16)$ & 12 \\
\hline & & 55201 & 1931 & $-10.64(0.56)$ & $-13.12(0.25)$ & 12 \\
\hline & & 55421 & 2151 & non & $-12.95(0.16)$ & 12 \\
\hline SN 2005A & Ia & 53387 & 12 & $-18.42(0.35)$ & $-17.95(0.35)$ & This work \\
\hline SN 2005W & Ia & 53607 & 205 & $-13.94(0.56)$ & $-14.01(0.77)$ & This work \\
\hline \multirow[t]{2}{*}{ SN 2005ad } & $\mathrm{II}-\mathrm{P}$ & 53605 & 198 & $-15.32(0.83)$ & $-15.96(0.83)$ & 8 \\
\hline & & 53771 & 364 & $-14.19(0.87)$ & $-15.30(0.85)$ & 8 \\
\hline \multirow[t]{5}{*}{ SN 2005af } & $\mathrm{II}-\mathrm{P}$ & 53573 & 194 & $-15.82(0.27)$ & $-17.97(0.27)$ & 8 \\
\hline & & 53779 & 399 & $-14.31(0.27)$ & $-16.14(0.27)$ & 8 \\
\hline & & 53955 & 576 & $-12.87(0.29)$ & $-14.06(0.29)$ & 8 \\
\hline & & 54151 & 772 & $-12.19(0.30)$ & $-12.45(0.31)$ & 8 \\
\hline & & 54320 & 940 & non & $-12.25(0.30)$ & 8 \\
\hline SN 2005at & Ic & 54003 & 569 & $-13.02(0.39)$ & $-13.85(0.30)$ & 13 \\
\hline \multirow[t]{3}{*}{$S N 2005 c p$} & IIn & 55065 & 1523 & $-20.53(0.19)$ & $-21.10(0.18)$ & 14 \\
\hline & & 55796 & 2254 & $-20.23(0.21)$ & $-20.51(0.20)$ & 15 \\
\hline & & 56542 & 3000 & $-20.14(0.21)$ & $-20.28(0.20)$ & This work \\
\hline \multirow[t]{2}{*}{ SN 2005df } & $\mathrm{Ia}$ & 53676 & 90 & $-15.99(0.53)$ & $-14.72(0.57)$ & 16 \\
\hline & & 53773 & 187 & $-14.34(0.57)$ & $-13.41(0.68)$ & 16 \\
\hline
\end{tabular}

Table A3 continued on next page 
Spitzer $\mathrm{SNE}$

Table A3 (continued)

\begin{tabular}{|c|c|c|c|c|c|c|}
\hline \multirow[t]{2}{*}{ Object } & \multirow[t]{2}{*}{ Type } & \multirow{2}{*}{$\begin{array}{l}\text { MJD - } \\
2450000\end{array}$} & \multirow{2}{*}{$\begin{array}{l}\text { Epoch } \\
\text { (days) }\end{array}$} & \multicolumn{2}{|c|}{ Absolute mag. } & \multirow{2}{*}{$\begin{array}{c}\text { Source of } \\
\text { data }^{\dagger}\end{array}$} \\
\hline & & & & $3.6 \mu \mathrm{m}$ & $4.5 \mu \mathrm{m}$ & \\
\hline & & 53955 & 369 & $-12.66(0.74)$ & $-12.35(0.90)$ & 16 \\
\hline \multirow[t]{3}{*}{ SN 2005gj } & Ia-CSM & 53778 & 139 & $-20.40(0.19)$ & $-20.99(0.20)$ & 5 \\
\hline & & 54004 & 365 & $-20.73(0.18)$ & $-21.18(0.19)$ & 5 \\
\hline & & 54149 & 510 & $-21.22(0.19)$ & $-21.83(0.18)$ & 5 \\
\hline SN 2005gn & IIn & 55065 & 1479 & $-20.08(0.42)$ & $-20.65(0.38)$ & 14 \\
\hline \multirow[t]{5}{*}{$S N 2005 i p$} & IIn & 54627 & 948 & $-20.70(0.52)$ & $-21.42(0.52)$ & 17 \\
\hline & & 55736 & 2057 & $-19.88(0.52)$ & $-20.75(0.52)$ & 15 \\
\hline & & $56475^{a}$ & 2796 & $-19.17(0.52)$ & $-20.02(0.52)$ & This work \\
\hline & & $56844^{b}$ & 3165 & $-18.93(0.52)$ & $-19.74(0.52)$ & This work \\
\hline & & $57228^{c}$ & 3549 & $-18.73(0.52)$ & $-19.47(0.52)$ & This work \\
\hline SN 2006E & Ia & 53955 & 208 & $-13.13(0.35)$ & $-12.99(0.45)$ & This work \\
\hline SN 2006X & Ia & 53922 & 152 & $-15.27(0.45)$ & $-14.13(0.54)$ & 18 \\
\hline SN 2006bc & $\mathrm{II}-\mathrm{P}$ & 54356 & 537 & $-13.55(0.78)$ & $-14.13(0.73)$ & 19 \\
\hline \multirow[t]{4}{*}{$S N 2006 b p$} & $I I-P$ & 54235 & 401 & $-15.60(0.44)$ & $-16.20(0.45)$ & 8 \\
\hline & & 54462 & 628 & $-15.40(0.44)$ & $-15.65(0.45)$ & 8 \\
\hline & & 54600 & 767 & $-15.36(0.45)$ & $-15.69(0.45)$ & 8 \\
\hline & & 55195 & 1361 & $-15.35(0.45)$ & $-15.54(0.47)$ & 8 \\
\hline SN 2006ce & Ia & 53955 & 90 & $-15.29(0.40)$ & $-14.32(0.47)$ & This work \\
\hline \multirow[t]{2}{*}{ SN 2006jc } & Ibn & 54227 & 210 & $-17.46(0.48)$ & $-18.23(0.47)$ & 20 \\
\hline & & 54429 & 412 & $-15.20(0.53)$ & $-16.23(0.50)$ & 20 \\
\hline \multirow[t]{4}{*}{$S N 2006 j d$} & IIn & 55169 & 1149 & $-21.40(0.14)$ & $-22.17(0.14)$ & 17 \\
\hline & & 55734 & 1714 & $-20.68(0.14)$ & $-21.62(0.14)$ & 21 \\
\hline & & $56453^{a}$ & 2433 & $-19.68(0.17)$ & $-20.74(0.15)$ & This work \\
\hline & & $56820^{b}$ & 2800 & $-19.30(0.18)$ & $-20.31(0.16)$ & This work \\
\hline SN 2006mq & Ia & 54226 & 196 & $-13.71(0.29)$ & $-11.94(0.79)$ & 1 \\
\hline \multirow[t]{3}{*}{ SN 2006my } & $\mathrm{II}-\mathrm{P}$ & 54149 & 205 & $-17.18(0.59)$ & $-17.49(0.59)$ & 8 \\
\hline & & 54285 & 342 & $-16.95(0.94)$ & $-17.02(0.58)$ & 8 \\
\hline & & 54503 & 559 & $-16.87(0.59)$ & $-16.91(0.59)$ & 8 \\
\hline \multirow[t]{2}{*}{ SN 2006ov } & $\mathrm{II}-\mathrm{P}$ & 54285 & 321 & $-15.43(0.55)$ & $-15.98(0.54)$ & 8 \\
\hline & & 54503 & 539 & $-15.36(0.55)$ & $-15.84(0.54)$ & 8 \\
\hline \multirow[t]{3}{*}{$S N 2006 q q$} & IIn & 55117 & 1048 & $-19.88(0.13)$ & $-20.70(0.11)$ & 14 \\
\hline & & 55874 & 1805 & $-19.07(0.23)$ & $-20.07(0.15)$ & 15 \\
\hline & & $56403^{a}$ & 2334 & $-18.95(0.23)$ & $-19.50(0.22)$ & This work \\
\hline SN 2007af & Ia & 54324 & 164 & $-15.13(0.59)$ & $-13.85(0.75)$ & 18 \\
\hline SN 2007gr & Ic & 54357 & 30 & $-18.00(0.96)$ & $-18.22(0.96)$ & 22 \\
\hline \multirow[t]{6}{*}{ SN 2007it } & $\mathrm{II}-\mathrm{P}$ & 54699 & 343 & $-15.20(0.30)$ & $-16.67(0.28)$ & 23 \\
\hline & & 54909 & 553 & $-16.04(0.28)$ & $-16.65(0.28)$ & 23 \\
\hline & & 55066 & 710 & $-15.05(0.30)$ & $-16.07(0.29)$ & 23 \\
\hline & & 55290 & 934 & $-13.13(0.50)$ & $-15.00(0.32)$ & This work \\
\hline & & 55441 & 1085 & non & $-14.50(0.35)$ & This work \\
\hline & & 55663 & 1307 & non & $-14.18(0.38)$ & This work \\
\hline SN 2007le & Ia & 54464 & 78 & $-16.19(0.33)$ & $-14.83(0.38)$ & 18 \\
\hline
\end{tabular}

Table A3 continued on next page 
Szalai ET AL.

Table A3 (continued)

\begin{tabular}{|c|c|c|c|c|c|c|}
\hline \multirow[t]{2}{*}{ Object } & \multirow[t]{2}{*}{ Type } & \multirow{2}{*}{$\begin{array}{l}\text { MJD - } \\
2450000\end{array}$} & \multirow{2}{*}{$\begin{array}{l}\text { Epoch } \\
\text { (days) }\end{array}$} & \multicolumn{2}{|c|}{ Absolute mag. } & \multirow{2}{*}{$\begin{array}{c}\text { Source of } \\
\text { data }^{\dagger}\end{array}$} \\
\hline & & & & $3.6 \mu \mathrm{m}$ & $4.5 \mu \mathrm{m}$ & \\
\hline \multirow[t]{4}{*}{ SN 2007oc } & \multirow[t]{4}{*}{$\mathrm{II}-\mathrm{P}$} & 54659 & 251 & $-16.91(0.40)$ & $-17.94(0.40)$ & 8 \\
\hline & & 54823 & 415 & $-16.35(0.40)$ & $-16.88(0.40)$ & 8 \\
\hline & & 55043 & 634 & $-15.57(0.42)$ & $-14.55(0.48)$ & 8 \\
\hline & & 55168 & 759 & $-15.81(0.42)$ & $-15.33(0.44)$ & 8 \\
\hline \multirow[t]{4}{*}{ SN 2007od } & \multirow[t]{4}{*}{$\mathrm{II}-\mathrm{P}$} & 54698 & 292 & $-16.13(0.31)$ & $-16.99(0.29)$ & 24 \\
\hline & & 54859 & 453 & $-14.98(0.39)$ & $-16.02(0.33)$ & 24 \\
\hline & & 55065 & 659 & $-14.48(0.43)$ & $-14.48(0.51)$ & 24 \\
\hline & & 55821 & 1415 & $-13.37(0.64)$ & $-13.04(0.90)$ & This work \\
\hline \multirow[t]{3}{*}{$S N 2007 r t$} & \multirow[t]{3}{*}{ IIn } & 55203 & 780 & $-21.74(0.19)$ & $-22.43(0.18)$ & 14 \\
\hline & & 55733 & 1304 & $-21.76(0.18)$ & $-22.16(0.18)$ & 15 \\
\hline & & $56465^{a}$ & 2042 & $-19.15(0.25)$ & $-19.94(0.23)$ & This work \\
\hline SN 2007sq & $\mathrm{II}-\mathrm{P}$ & 54596 & 154 & $-17.41(0.82)$ & $\ldots$ & This work \\
\hline SN 2007sr & Ia & 54528 & 76 & $-16.43(0.44)$ & $-15.08(0.50)$ & 18 \\
\hline SN 2008J & IIn & 55073 & 593 & $-21.25(0.17)$ & $-21.88(0.16)$ & 15 \\
\hline SN 2008Q & Ia & 54727 & 236 & $-13.70(0.65)$ & $-13.91(0.69)$ & 25 \\
\hline \multirow[t]{2}{*}{ SN $2008 \mathrm{cg}$} & \multirow[t]{2}{*}{ IIn } & 55065 & 474 & $-20.32(0.37)$ & $-21.01(0.37)$ & 14 \\
\hline & & 55817 & 1226 & $-16.70(0.70)$ & $-18.33(0.58)$ & 15 \\
\hline \multirow[t]{3}{*}{ SN 2008en } & \multirow[t]{3}{*}{ IIn } & 55067 & 386 & $-20.73(0.13)$ & $-21.38(0.13)$ & 14 \\
\hline & & 55812 & 1131 & $-19.31(0.23)$ & $-20.26(0.19)$ & 15 \\
\hline & & $56378^{a}$ & 1697 & $-18.61(0.31)$ & $-19.56(0.26)$ & This work \\
\hline SN 2008fq & II & 55892 & 1168 & $-15.44(0.68)$ & $-16.26(0.51)$ & This work \\
\hline \multirow[t]{3}{*}{ SN 2008gm } & \multirow[t]{3}{*}{ IIn } & 55062 & 301 & $-17.55(0.23)$ & $-17.76(0.24)$ & 14 \\
\hline & & 55796 & 1035 & $-17.58(0.22)$ & $-17.66(0.25)$ & 15 \\
\hline & & $56531^{a}$ & 1770 & $-17.53(0.23)$ & $-17.60(0.26)$ & This work \\
\hline SN 2008ip & IIn & 55088 & 438 & $-17.13(0.67)$ & $-17.97(0.49)$ & 14 \\
\hline \multirow[t]{2}{*}{ SN 2008jb } & \multirow[t]{2}{*}{ II } & 55076 & 287 & $-15.48(0.68)$ & $-16.63(0.68)$ & 26 \\
\hline & & 55113 & 324 & $-15.04(0.68)$ & $-16.18(0.68)$ & 26 \\
\hline SN $2009 \mathrm{E}$ & $\mathrm{II}-\mathrm{P}$ & 55381 & 547 & $-14.25(0.43)$ & $-16.15(0.11)$ & This work \\
\hline \multirow[t]{2}{*}{$S N 2009 H$} & \multirow[t]{2}{*}{$I I$} & 55077 & 243 & $-17.79(0.06)$ & $-18.68(0.06)$ & This work \\
\hline & & 55116 & 282 & $-17.54(0.07)$ & $-18.53(0.06)$ & This work \\
\hline \multirow[t]{2}{*}{ SN 2009af } & \multirow[t]{2}{*}{$I I$} & 55077 & 199 & $-16.85(0.40)$ & $-18.14(0.38)$ & This work \\
\hline & & 55107 & 229 & $-16.34(0.40)$ & $-17.76(0.38)$ & This work \\
\hline SN 2009at & $I I$ & 55375 & 474 & $16.01(0.42)$ & $-16.56(0.42)$ & This work \\
\hline & & 55406 & 505 & $-15.93(0.42)$ & $-16.51(0.42)$ & This work \\
\hline SN $2009 \mathrm{em}$ & Ic & 55073 & 117 & $-14.54(0.67)$ & $-16.40(0.65)$ & This work \\
\hline SN 2009gj & IIb & 55070 & 68 & $-16.01(0.42)$ & $-17.16(0.41)$ & This work \\
\hline SN 2009ig & Ia & 55076 & 13 & $-18.59(0.11)$ & $-18.79(0.12)$ & 18 \\
\hline & & 55115 & 52 & $-17.33(0.16)$ & $-16.45(0.27)$ & 18 \\
\hline$S N$ 2009ip $p^{\ddagger}$ & IIn & 56323 & 130 & $-15.72(0.26)$ & $-16.40(0.25)$ & 27 \\
\hline & & 56498 & 305 & $-14.08(0.39)$ & $-15.02(0.34)$ & 27 \\
\hline & & 56518 & 325 & $-14.05(0.40)$ & $-14.95(0.35)$ & 27 \\
\hline & & 56531 & 338 & $-13.94(0.41)$ & $-14.88(0.36)$ & 27 \\
\hline
\end{tabular}

Table A3 continued on next page 
Spitzer $\mathrm{SNE}$

Table A3 (continued)

\begin{tabular}{|c|c|c|c|c|c|c|}
\hline \multirow[t]{2}{*}{ Object } & \multirow[t]{2}{*}{ Type } & \multirow{2}{*}{$\begin{array}{l}\text { MJD - } \\
2450000\end{array}$} & \multirow{2}{*}{$\begin{array}{l}\text { Epoch } \\
\text { (days) }\end{array}$} & \multicolumn{2}{|c|}{ Absolute mag. } & \multirow{2}{*}{$\begin{array}{c}\text { Source of } \\
\text { data }^{\dagger}\end{array}$} \\
\hline & & & & $3.6 \mu \mathrm{m}$ & $4.5 \mu \mathrm{m}$ & \\
\hline & & $56905^{b}$ & 712 & $-12.43(0.75)$ & $-13.640 .56)$ & This work \\
\hline & & $57278^{c}$ & 1085 & $-12.03(0.90)$ & $-13.32(0.64)$ & This work \\
\hline & & $57617^{d}$ & 1424 & $-12.08(0.88)$ & $-13.06(0.71)$ & This work \\
\hline SN 2009iu & $I b$ & 55142 & 67 & $-17.55(0.31)$ & $-18.48(0.30)$ & This work \\
\hline SN 2009jf & $\mathrm{Ib}$ & 55201 & 100 & $-16.86(0.46)$ & $-17.92(0.46)$ & This work \\
\hline \multirow[t]{3}{*}{ SN 2009jr } & $\mathrm{Ia}$ & 55150 & 38 & $-17.45(0.18)$ & non & This work \\
\hline & & 55161 & 49 & $-17.25(0.18)$ & non & This work \\
\hline & & 55171 & 59 & $-16.52(0.18)$ & non & This work \\
\hline SN 2009js & II-P p. & 55117 & 2 & $-15.95(0.76)$ & $-16.20(0.76)$ & 28 \\
\hline $\mathrm{SN} 2009 \mathrm{mk}$ & $\mathrm{IIb}$ & 55405 & 225 & $-15.09(0.20)$ & $-15.94(0.20)$ & This work \\
\hline \multirow[t]{4}{*}{$S N 2010 B$} & $I a$ & 55246 & 43 & $-17.16(0.24)$ & $-16.68(0.32)$ & This work \\
\hline & & 55281 & 78 & $-16.71(0.27)$ & $-16.26(0.38)$ & This work \\
\hline & & 55311 & 108 & $-16.52(0.29)$ & $-16.19(0.39)$ & This work \\
\hline & & 55343 & 140 & $-16.47(0.29)$ & $-16.35(0.36)$ & This work \\
\hline \multirow[t]{3}{*}{ SN 2010F } & II & 55416 & 207 & $-15.46(0.66)$ & $-17.10(0.54)$ & This work \\
\hline & & 55587 & 378 & $-13.93(1.51)$ & $-15.40(0.74)$ & This work \\
\hline & & 55740 & 531 & non & $-13.65(1.20)$ & This work \\
\hline \multirow[t]{8}{*}{ SN 2010jl } & IIn & 55570 & 67 & $-21.40(0.15)$ & $-21.91(0.15)$ & 29 \\
\hline & & 55732 & 229 & $-21.27(0.15)$ & $-21.87(0.15)$ & 30 \\
\hline & & 55943 & 440 & $-22.14(0.15)$ & $-22.60(0.15)$ & 30 \\
\hline & & 56099 & 596 & $-22.16(0.15)$ & $-22.64(0.15)$ & 30 \\
\hline & & 56322 & 819 & $-22.04(0.15)$ & $-22.58(0.15)$ & 30 \\
\hline & & 56472 & 969 & $-22.23(0.15)$ & $-22.83(0.15)$ & This work \\
\hline & & 56846 & 1343 & $-21.53(0.15)$ & $-22.31(0.15)$ & This work \\
\hline & & $57228^{c}$ & 1725 & $-20.76(0.15)$ & $-21.75(0.15)$ & This work \\
\hline SN 2010gp & Ia & 55498 & 107 & $-16.60(0.38)$ & $-16.62(0.49)$ & This work \\
\hline \multirow[t]{2}{*}{$S N 2010 m c$} & IIn & 56814 & 1386 & $-18.78(0.33)$ & $-19.32(0.33)$ & This work \\
\hline & & 57163 & 1735 & $-18.68(0.35)$ & $-19.22(0.34)$ & This work \\
\hline SN 2011A & IIn & 55649 & 86 & $-16.04(0.39)$ & $-16.32(0.36)$ & This work \\
\hline SN 2011ae & Ia & 55616 & 12 & $-18.62(0.05)$ & $-18.71(0.06)$ & This work \\
\hline \multirow[t]{12}{*}{ SN 2011dh } & $\mathrm{IIb}$ & 55730 & 17 & $-17.71(0.11)$ & $-18.10(0.10)$ & 31 \\
\hline & & 55736 & 23 & $-17.90(0.11)$ & $-18.27(0.11)$ & 31 \\
\hline & & 55743 & 30 & $-17.89(0.11)$ & $-18.27(0.11)$ & 31 \\
\hline & & 55750 & 37 & $-17.86(0.11)$ & $-18.28(0.11)$ & 31 \\
\hline & & 55758 & 44 & $-17.76(0.11)$ & $-18.27(0.11)$ & 31 \\
\hline & & 55765 & 52 & $-17.60(0.11)$ & $-18.23(0.11)$ & 31 \\
\hline & & 55771 & 58 & $-17.44(0.11)$ & $-18.19(0.11)$ & 31 \\
\hline & & 55778 & 65 & $-17.26(0.11)$ & $-18.14(0.11)$ & 31 \\
\hline & & 55785 & 71 & $-17.07(0.11)$ & $-18.07(0.11)$ & 31 \\
\hline & & 55797 & 84 & $-16.72(0.11)$ & $-17.94(0.11)$ & 31 \\
\hline & & 55963 & 250 & $-15.18(0.11)$ & $-16.19(0.11)$ & 31 \\
\hline & & 55993 & 280 & $-15.01(0.11)$ & $-16.47(0.11)$ & 31 \\
\hline
\end{tabular}

Table A3 continued on next page 
SzALAI ET AL.

Table A3 (continued)

\begin{tabular}{|c|c|c|c|c|c|c|}
\hline \multirow[t]{2}{*}{ Object } & \multirow[t]{2}{*}{ Type } & \multirow{2}{*}{$\begin{array}{c}\text { MJD - } \\
2450000\end{array}$} & \multirow{2}{*}{$\begin{array}{l}\text { Epoch } \\
\text { (days) }\end{array}$} & \multicolumn{2}{|c|}{ Absolute mag. } & \multirow{2}{*}{$\begin{array}{c}\text { Source of } \\
\operatorname{data}^{\dagger}\end{array}$} \\
\hline & & & & $3.6 \mu \mathrm{m}$ & $4.5 \mu \mathrm{m}$ & \\
\hline & & 56026 & 312 & $-13.98(0.12)$ & $-15.27(0.11)$ & 31 \\
\hline & & 56103 & 390 & $-12.43(0.28)$ & $-14.00(0.14)$ & 31 \\
\hline & & 56135 & 422 & $-12.12(0.37)$ & $-13.55(0.18)$ & 31 \\
\hline & & 56168 & 454 & $-11.88(0.45)$ & $-13.29(0.21)$ & 31 \\
\hline & & 56337 & 623 & $-10.95(1.04)$ & $-12.01(0.61)$ & 32 \\
\hline SN 2011dq & II & 56955 & 1259 & $-18.20(0.28)$ & $-18.41(0.27)$ & This work \\
\hline SN 2011dx & $\mathrm{Ia}-$ pec. & 55864 & 125 & $-15.02(0.55)$ & non & This work \\
\hline \multirow[t]{6}{*}{ SN 2011fe } & Ia & 55961 & 145 & $-14.87(0.17)$ & $-13.73(0.18)$ & 25 \\
\hline & & 55981 & 165 & $-14.55(0.18)$ & $-13.44(0.18)$ & 25 \\
\hline & & 56048 & 233 & $-13.61(0.18)$ & $-13.02(0.19)$ & 25 \\
\hline & & 56165 & 349 & $-11.77(0.24)$ & $-11.75(0.30)$ & 25 \\
\hline & & 56337 & 522 & non & $-11.16(0.50)$ & 25 \\
\hline & & 56348 & 533 & non & $-11.27(0.39)$ & 25 \\
\hline SN 2011fh & IIn & 56383 & 586 & $-15.07(0.56)$ & $-16.74(0.45)$ & This work \\
\hline SN 2011ft & $I b$ & 56060 & 257 & $-18.68(0.21)$ & $\ldots$ & This work \\
\hline SN 2011ht & IIn & 55833 & 638 & non & $-12.90(0.74)$ & This work \\
\hline SN 2011ir & IIn & 55886 & 832 & $-16.98(0.48)$ & $-17.78(0.44)$ & This work \\
\hline SN 2011iy & Ia & 56932 & 1028 & $\ldots$ & $-12.79(0.36)$ & This work \\
\hline \multirow[t]{11}{*}{ SN 2011ja } & $\mathrm{II}-\mathrm{P}$ & 56012 & 106 & $-16.22(0.28)$ & $-16.99(0.28)$ & 33 \\
\hline & & 56393 & 488 & $-16.25(0.28)$ & $-17.07(0.28)$ & 33 \\
\hline & & 56544 & 639 & $-15.48(0.28)$ & $-16.47(0.28)$ & 33 \\
\hline & & 56764 & 859 & $-14.37(0.30)$ & $-15.70(0.29)$ & 33 \\
\hline & & 56787 & 882 & $-14.21(0.31)$ & $-15.56(0.29)$ & 1 \\
\hline & & 56800 & 895 & $-14.13(0.32)$ & $-15.44(0.29)$ & 1 \\
\hline & & 56912 & 1007 & $-13.36(0.35)$ & $-15.00(0.29)$ & 1 \\
\hline & & 57136 & 1231 & $\ldots$ & $-14.39(0.29)$ & 1 \\
\hline & & 57143 & 1238 & $\ldots$ & $-14.27(0.29)$ & 1 \\
\hline & & 57164 & 1259 & $\ldots$ & $-14.04(0.29)$ & 1 \\
\hline & & 57288 & 1382 & non & $-13.22(0.28)$ & 1 \\
\hline \multirow[t]{2}{*}{$P T F 11 k x$} & $I a-C S M$ & 56816 & 1237 & $-19.63(0.48)$ & $-20.31(0.58)$ & 34 \\
\hline & & 57397 & 1818 & $-18.64(1.11)$ & $-19.56(1.11)$ & 34 \\
\hline \multirow[t]{2}{*}{ PTF11qcj } & Ic & 56014 & 174 & $-17.53(0.61)$ & $-18.31(0.48)$ & 35 \\
\hline & & 56103 & 263 & $-16.42(0.84)$ & $-17.34(0.57)$ & 35 \\
\hline \multirow[t]{2}{*}{ SN 2012aw } & $\mathrm{II}-\mathrm{P}$ & 56360 & 358 & $-13.93(0.25)$ & $-15.88(0.25)$ & This work \\
\hline & & 56489 & 487 & $-12.80(0.33)$ & $-14.70(0.26)$ & This work \\
\hline \multirow[t]{3}{*}{$S N 2012 c a$} & $I a-C S M$ & 56446 & 404 & $-20.68(0.18)$ & $-21.34(0.17$ & This work \\
\hline & & 56619 & 577 & $-20.89(0.17)$ & $-21.71(0.17)$ & This work \\
\hline & & 56820 & 778 & $-19.69(0.19)$ & $-21.02(0.18)$ & This work \\
\hline$S N$ 2012cd & $I I b$ & 5609 & 37 & $-18.56(0.19)$ & $-19.07(0.19)$ & This work \\
\hline \multirow[t]{3}{*}{ SN 2012cg } & Ia & 56140 & 58 & $-16.44(0.24)$ & $-15.58(0.25)$ & 18 \\
\hline & & 56153 & 71 & $-16.30(0.24)$ & $-15.34(0.26)$ & 18 \\
\hline & & 56163 & 81 & $-16.04(0.24)$ & $-15.07(0.27)$ & 18 \\
\hline
\end{tabular}

Table A3 continued on next page 
Spitzer $\mathrm{SNE}$

Table A3 (continued)

\begin{tabular}{|c|c|c|c|c|c|c|}
\hline \multirow[t]{2}{*}{ Object } & \multirow[t]{2}{*}{ Type } & \multirow{2}{*}{$\begin{array}{l}\text { MJD - } \\
2450000\end{array}$} & \multirow{2}{*}{$\begin{array}{l}\text { Epoch } \\
\text { (days) }\end{array}$} & \multicolumn{2}{|c|}{ Absolute mag. } & \multirow{2}{*}{$\begin{array}{c}\text { Source of } \\
\text { data }^{\dagger}\end{array}$} \\
\hline & & & & $3.6 \mu \mathrm{m}$ & $4.5 \mu \mathrm{m}$ & \\
\hline & & 56175 & 93 & $-15.88(0.25)$ & $-14.92(0.28)$ & 18 \\
\hline \multirow[t]{5}{*}{ SN 2012fh } & Ic & 56117 & 8 & $-17.64(0.44)$ & $-17.92(0.44)$ & This work \\
\hline & & 56122 & 13 & $-17.74(0.44)$ & $-17.98(0.44)$ & This work \\
\hline & & 56323 & 214 & $-16.40(0.44)$ & $-17.52(0.44)$ & This work \\
\hline & & 56356 & 247 & $-15.87(0.45)$ & $-17.10(0.45)$ & This work \\
\hline & & 56692 & 583 & non & $-12.39(0.95)$ & This work \\
\hline$S N$ 2013E & $I a$ & 56376 & 80 & $-16.17(0.57)$ & $\ldots$ & This work \\
\hline \multirow[t]{2}{*}{$S N 2013 L$} & IIn & 56944 & 630 & $-20.44(0.15)$ & $-21.08(0.15)$ & 36 \\
\hline & & 57164 & 850 & $-20.34(0.16)$ & $-21.04(0.15)$ & 36 \\
\hline \multirow[t]{4}{*}{ SN 2013ai } & II & 56671 & 319 & $-14.64(1.35)$ & $\ldots$ & 1 \\
\hline & & 56790 & 448 & $\ldots$ & $-14.77(1.36)$ & 1 \\
\hline & & 56816 & 474 & non & $-14.59(1.37)$ & 1 \\
\hline & & 57057 & 705 & $-12.08(1.21)$ & $-14.20(1.14)$ & 1 \\
\hline \multirow[t]{3}{*}{ SN 2013am } & II & 56715 & 343 & $-13.70(0.50)$ & $-14.10(0.49)$ & 1 \\
\hline & & 56741 & 369 & $-13.44(0.55)$ & $-14.45(0.45)$ & 1 \\
\hline & & 56865 & 493 & $-11.28(1.15)$ & $-13.39(0.64)$ & 1 \\
\hline \multirow[t]{2}{*}{ SN 2013bu } & II & 56690 & 287 & $-14.86(0.47)$ & $-15.90(0.42)$ & 1 \\
\hline & & 56905 & 502 & non & $-14.22(0.41)$ & 1 \\
\hline \multirow[t]{2}{*}{$S N 2013 c j$} & IIn & 56815 & 394 & $-22.04(0.17)$ & $-22.42(0.17)$ & This work \\
\hline & & 57168 & 747 & $-22.14(0.17)$ & $-22.64(0.17)$ & This work \\
\hline \multirow[t]{5}{*}{ SN 2013df } & $\mathrm{IIb}$ & 56715 & 268 & $-14.19(0.34)$ & $-15.48(0.34)$ & 1 \\
\hline & & 56742 & 294 & $-13.57(0.44)$ & $-15.17(0.37)$ & 1 \\
\hline & & 56871 & 423 & $-12.77(0.49)$ & $-14.00(0.33)$ & 1 \\
\hline & & 56906 & 459 & $-12.69(0.52)$ & $-13.87(0.35)$ & 1 \\
\hline & & 57272 & 824 & $-12.59(0.55)$ & $-13.48(0.42)$ & 1 \\
\hline \multirow[t]{6}{*}{ SN 2013dk } & Ic & 56733 & 268 & $-16.05(0.40)$ & $-16.79(0.40)$ & 1 \\
\hline & & 56734 & 269 & $-15.89(0.40)$ & $-16.80(0.40)$ & 1 \\
\hline & & 56758 & 292 & $-15.62(0.41)$ & $-16.48(0.40)$ & 1 \\
\hline & & 56763 & 298 & $-15.71(0.41)$ & $-16.42(0.41)$ & 1 \\
\hline & & 56894 & 428 & $-14.85(0.41)$ & $-15.51(0.42)$ & 1 \\
\hline & & 57109 & 644 & non & $-13.83(0.44)$ & 1 \\
\hline \multirow[t]{2}{*}{$S N 2013 d n$} & $I a-C S M$ & 56920 & 463 & $-20.70(0.24)$ & $-21.46(0.24)$ & This work \\
\hline & & 57285 & 828 & $-19.66(0.32)$ & $-20.36(0.31)$ & This work \\
\hline \multirow[t]{2}{*}{ SN 2013dy } & Ia & 56691 & 208 & $-13.42(0.72)$ & $-12.25(0.75)$ & This work \\
\hline & & 56731 & 248 & $-12.72(0.73)$ & $-12.25(0.75)$ & This work \\
\hline \multirow[t]{3}{*}{ SN 2013ee } & II & 56677 & 191 & $-14.95(0.68)$ & $-16.78(0.61)$ & This work \\
\hline & & 56706 & 220 & $-15.33(0.63)$ & $-16.71(0.62)$ & This work \\
\hline & & 56827 & 341 & $-14.81(0.71)$ & $-15.85(0.63)$ & This work \\
\hline \multirow[t]{4}{*}{ SN 2013ej } & $\mathrm{II}-\mathrm{P} / \mathrm{L}$ & 56735 & 238 & $-15.87(0.18)$ & $-17.02(0.14)$ & 37 \\
\hline & & 56758 & 261 & $-15.58(0.18)$ & $-16.76(0.13)$ & 37 \\
\hline & & 56937 & 439 & $-12.62(0.22)$ & $-14.37(0.17)$ & 37 \\
\hline & & 56965 & 468 & $-12.11(0.41)$ & $-13.98(0.21)$ & 37 \\
\hline
\end{tabular}

Table A3 continued on next page 
Szalai ET AL.

Table A3 (continued)

\begin{tabular}{|c|c|c|c|c|c|c|}
\hline \multirow[t]{2}{*}{ Object } & \multirow[t]{2}{*}{ Type } & \multirow{2}{*}{$\begin{array}{l}\text { MJD - } \\
2450000\end{array}$} & \multirow{2}{*}{$\begin{array}{l}\text { Epoch } \\
\text { (days) }\end{array}$} & \multicolumn{2}{|c|}{ Absolute mag. } & \multirow{2}{*}{$\begin{array}{c}\text { Source of } \\
\text { data }^{\dagger}\end{array}$} \\
\hline & & & & $3.6 \mu \mathrm{m}$ & $4.5 \mu \mathrm{m}$ & \\
\hline & & 56970 & 473 & $-12.20(0.16)$ & $-13.81(0.21)$ & 37 \\
\hline & & 57321 & 823 & non & $-10.97(0.43)$ & 37 \\
\hline & & 57334 & 837 & non & $-10.88(0.47)$ & 37 \\
\hline & & 57475 & 978 & non & $-11.20(0.35)$ & 37 \\
\hline & & 57482 & 985 & non & $-11.81(0.24)$ & 37 \\
\hline & & 57504 & 1006 & non & $-11.89(0.21)$ & 37 \\
\hline & & $57680^{e}$ & 1182 & non & $-11.58(0.58)$ & This work \\
\hline & & $57695^{f}$ & 1197 & non & $-11.70(0.52)$ & This work \\
\hline & & $57855^{e}$ & 1357 & non & $-11.32(0.45)$ & This work \\
\hline SN 2013ff & Ic & 56682 & 147 & $-16.46(0.40)$ & $-17.89(0.31)$ & This work \\
\hline \multirow[t]{9}{*}{ SN 2014C } & $\mathrm{Ib}$ & 56707 & 45 & $-18.84(0.37)$ & $-19.66(0.37)$ & 1 \\
\hline & & 56905 & 243 & $-18.18(0.37)$ & $-18.91(0.37)$ & 1 \\
\hline & & 56937 & 274 & $-18.34(0.37)$ & $-19.04(0.37)$ & 1 \\
\hline & & 57284 & 622 & $-18.57(0.37)$ & $-19.44(0.37)$ & 1 \\
\hline & & 57290 & 628 & $-18.49(0.37)$ & $-19.30(0.37)$ & 1 \\
\hline & & 57304 & 642 & $-18.50(0.37)$ & $-19.47(0.37)$ & 1 \\
\hline & & 57435 & 772 & $-18.22(0.37)$ & $-19.23(0.37)$ & 1 \\
\hline & & 57442 & 780 & $-18.18(0.37)$ & $-19.22(0.37)$ & 1 \\
\hline & & 57464 & 801 & $-18.15(0.37)$ & $-19.22(0.37)$ & 1 \\
\hline SN 2014G & $\mathrm{II}-\mathrm{L}$ & 57232 & 561 & $-14.86(0.31)$ & $-16.13(0.26)$ & This work \\
\hline \multirow[t]{10}{*}{ SN 2014J } & Ia & 56685 & 8 & non & $-18.61(0.13)$ & 18 \\
\hline & & 56695 & 17 & $-18.00(0.13)$ & $-17.90(0.13)$ & 18 \\
\hline & & 56700 & 23 & $-17.58(0.13)$ & $-17.10(0.13)$ & 18 \\
\hline & & 56707 & 29 & $-17.27(0.13)$ & $-16.68(0.13)$ & 18 \\
\hline & & 56712 & 34 & $-17.15(0.13)$ & $-16.48(0.13)$ & 18 \\
\hline & & 56718 & 41 & $-17.00(0.13)$ & $-16.32(0.13)$ & 18 \\
\hline & & 56807 & 129 & $-15.56(0.14)$ & $-14.40(0.16)$ & 18 \\
\hline & & 56816 & 138 & $-15.40(0.14)$ & $-14.25(0.17)$ & 18 \\
\hline & & 56831 & 154 & $-15.03(0.14)$ & $-13.99(0.19)$ & 18 \\
\hline & & 56846 & 168 & $-14.82(0.15)$ & $-13.67(0.23)$ & 18 \\
\hline \multirow[t]{3}{*}{ SN 2014L } & Ic & 56723 & 40 & $-17.50(0.28)$ & $-18.05(0.27)$ & 1 \\
\hline & & 56750 & 67 & $-17.02(0.28)$ & $-17.93(0.28)$ & 1 \\
\hline & & 56890 & 206 & $-15.53(0.28)$ & $-16.68(0.28)$ & 1 \\
\hline \multirow[t]{7}{*}{ SN 2014bi } & $\mathrm{II}-\mathrm{P}$ & 56847 & 38 & $-16.11(0.46)$ & $-16.32(0.46)$ & 1 \\
\hline & & 57072 & 264 & $-11.77(0.51)$ & $-14.74(0.51)$ & 1 \\
\hline & & 57076 & 268 & $-11.98(0.49)$ & $-14.68(0.51)$ & 1 \\
\hline & & 57080 & 272 & $-11.99(0.49)$ & $-14.68(0.51)$ & 1 \\
\hline & & 57102 & 293 & $-12.08(0.49)$ & $-14.49(0.53)$ & 1 \\
\hline & & 57253 & 445 & $-12.51(0.49)$ & $-13.86(0.83)$ & 1 \\
\hline & & $57484^{e}$ & 676 & $-10.95(1.18)$ & $-12.47(0.62)$ & This work \\
\hline SN 2014cx & $\mathrm{II}-\mathrm{P}$ & 56955 & 53 & $-18.19(0.27)$ & $-18.36(0.28)$ & This work \\
\hline SN 2014df & $\mathrm{Ib}$ & 56929 & 118 & $-15.55(0.38)$ & non & 1 \\
\hline
\end{tabular}

Table A3 continued on next page 
Spitzer SNE

Table A3 (continued)

\begin{tabular}{ccccccc}
\hline \hline Object & Type & MJD - & Epoch & \multicolumn{2}{c}{ Absolute mag. } & Source of \\
& & 2450000 & (days) & $3.6 \mu \mathrm{m}$ & $4.5 \mu \mathrm{m}$ & data $^{\dagger}$ \\
\hline SN 2014dt & Iax & 57259 & 309 & $-14.46(0.92)$ & $-15.01(0.88)$ & 38 \\
& & 57267 & 317 & $-14.53(0.92)$ & $-15.13(0.88)$ & 38 \\
& & 57286 & 336 & $-14.93(0.88)$ & $-15.38(0.87)$ & 38 \\
\hline
\end{tabular}

NoтE - ${ }^{\dagger}$ Fluxes, distances, epochs and $E(B-V)$ values originate from our current work or are adopted from the papers listed here. $\ddagger$. In the case of SN 2009ip, epochs are given regarding the SN-like outburst observed in 2012. Data marked with italic denote cases where image subtraction can not be applied. References: ${ }^{1}$ Tinyanont et al. (2016); ${ }^{2}$ Van Dyk (2013); ${ }^{3}$ Szczygiel et al. (2012); ${ }^{4}$ Meikle et al. (2006); ${ }^{5}$ Fox \& Filippenko (2013); ${ }^{6}$ Meikle et al. (2007); ${ }^{7}$ Meikle et al. (2005); ${ }^{8}$ Szalai \& Vinkó (2013); ${ }^{9}$ Szalai et al. (2011); ${ }^{10}$ Meikle et al. (2011); ${ }^{11}$ Kotak et al. (2009); ${ }^{12}$ Fabbri et al. (2011); ${ }^{13}$ Kankare et al. (2014); ${ }^{14}$ Fox et al. (2011); ${ }^{15}$ Fox et al. (2013); ${ }^{16}$ Gerardy et al. (2007); ${ }^{17}$ Fox et al. (2010); ${ }^{18}$ Johansson et al. (2017); ${ }^{19}$ Gallagher et al. (2012); ${ }^{20}$ Mattila et al. (2008); ${ }^{21}$ Stritzinger et al. (2012); ${ }^{22}$ Kochanek et al. (2011); ${ }^{23}$ Andrews et al. (2011b); ${ }^{24}$ Andrews et al. (2010); ${ }^{25}$ McClelland et al. (2013); ${ }^{26}$ Prieto et al. (2012); ${ }^{27}$ Fraser et al. (2015); ${ }^{28}$ Gandhi et al. (2013); ${ }^{29}$ Andrews et al. (2011a); ${ }^{30}$ Fransson et al. (2014); ${ }^{31}$ Helou et al. (2013); ${ }^{32}$ Ergon et al. (2015); ${ }^{33}$ Andrews et al. (2016); ${ }^{34}$ Graham et al. (2017); ${ }^{35}$ Corsi et al. (2014); ${ }^{36}$ Andrews et al. (2017); ${ }^{37}$ Mauerhan et al. (2017); ${ }^{38}$ Fox et al. (2016). Additional notes. Sources of new data of previously known positive Spitzer targets (PID): ${ }^{a} 90174$ (PI Fox); ${ }^{b} 10139$ (PI Fox); ${ }^{c} 11053$ (PI Fox); ${ }^{d} 12099$ (PI Fraser); 13053 (PI Kasliwal); ${ }^{f} 11063$ (PI Kasliwal). 
Table A4. Dust parameters of the studied SNe (pure graphite dust with a grain size of $a=0.1 \mu \mathrm{m}$ )

\begin{tabular}{|c|c|c|c|c|c|c|c|c|}
\hline Object & Type & $\begin{array}{l}\text { Epoch } \\
\text { (days) }\end{array}$ & $\begin{array}{c}R_{B B} \\
\left(10^{16} \mathrm{~cm}\right)\end{array}$ & $\begin{array}{c}v_{B B} \\
\left(\mathrm{~km} \mathrm{~s}^{-1}\right)\end{array}$ & $\begin{array}{c}T_{\text {dust }} \\
(\mathrm{K})\end{array}$ & $\begin{array}{c}M_{d u s t} \\
\left(10^{-5} \mathrm{M}_{\odot}\right)\end{array}$ & $\begin{array}{c}L_{d u s t} \\
\left(10^{6} L_{\odot}\right)\end{array}$ & Notes \\
\hline SN $1995 N$ & IIn & 5067 & 2.4 & 550 & 350 & $<478$ & $<3.8$ & Possible Si dust $\left(M=1.4 \times 10^{-3} M_{\odot}, T=410 K\right)$ \\
\hline SN 2001em & $I b / c$ & 1135 & 15.4 & 15700 & 280 & $<22800$ & $<58.4$ & + hot comp. $\left(R=10^{16} \mathrm{~cm}, T=1400 \mathrm{~K}\right)$ \\
\hline SN 2001gd & $\mathrm{IIb}$ & 952 & 0.06 & 80 & 730 & 0.21 & 0.1 & \\
\hline SN 2002bu & IIn & 759 & 0.4 & 640 & 520 & 10.8 & 0.7 & \\
\hline \multirow[t]{3}{*}{ SN 2002ic } & Ia-CSM & 795 & 8.5 & 12370 & 590 & 1200 & 160 & \\
\hline & & 1179 & 8.8 & 8640 & 495 & 1600 & 87.0 & \\
\hline & & 1370 & 8.0 & 6760 & 480 & 1400 & 63.0 & \\
\hline SN 2003gd & $\mathrm{II}-\mathrm{P}$ & 409 & 0.4 & 1020 & 520 & 1.1 & 0.1 & \\
\hline \multirow[t]{2}{*}{ SN 2003lo } & IIn & 256 & 0.6 & 2490 & 640 & 14.1 & 2.9 & \\
\hline & & 384 & 0.3 & 840 & 690 & 4.0 & 1.2 & \\
\hline \multirow[t]{3}{*}{ SN 2004A } & $\mathrm{II}-\mathrm{P}$ & 247 & 3.4 & 15930 & 240 & 2438 & 2.9 & + hot comp. $\left(\mathrm{R}=0.05 \times 10^{16} \mathrm{~cm}, \mathrm{~T}=4800 \mathrm{~K}\right)$ \\
\hline & & 445 & 3.8 & 9885 & 270 & 852 & 1.8 & \\
\hline & & 563 & 2.2 & 4520 & 300 & 386 & 1.4 & \\
\hline \multirow[t]{4}{*}{ SN 20004dj } & $\mathrm{II}-\mathrm{P}$ & 267 & 0.2 & 870 & 890 & 0.3 & 0.4 & + cold comp. $\left(\mathrm{R}=1.5 \times 10^{16} \mathrm{~cm}, \mathrm{~T}=186 \mathrm{~K}\right)$ \\
\hline & & 850 & 0.4 & 540 & 630 & 1.1 & 0.3 & + cold comp. $\left(\mathrm{R}=4.3 \times 10^{16} \mathrm{~cm}, \mathrm{~T}=120 \mathrm{~K}\right)$ \\
\hline & & 1006 & 0.4 & 460 & 550 & 1.3 & 0.2 & + cold comp. $\left(\mathrm{R}=4.6 \times 10^{16} \mathrm{~cm}, \mathrm{~T}=110 \mathrm{~K}\right)$ \\
\hline & & 1237 & 0.4 & 370 & 490 & 1.4 & 0.1 & + cold comp. $\left(\mathrm{R}=6.5 \times 10^{16} \mathrm{~cm}, \mathrm{~T}=103 \mathrm{~K}\right)$ \\
\hline \multirow[t]{4}{*}{ SN 2004et } & $\mathrm{II}-\mathrm{P}$ & 300 & 0.4 & 1600 & 550 & 10.0 & 0.9 & + Possible Si dust $\left(\mathrm{M}=3.8 \times 10^{-5} \mathrm{M}_{\odot}, \mathrm{T}=900 \mathrm{~K}\right)$ \\
\hline & & 360 & 0.4 & 1300 & 520 & 11.0 & 0.7 & + Possible Si dust $\left(\mathrm{M}=5.6 \times 10^{-5} \mathrm{M}_{\odot}, \mathrm{T}=730 \mathrm{~K}\right)$ \\
\hline & & 465 & 0.4 & 1100 & 480 & 8.2 & 0.3 & + Possible Si dust $\left(\mathrm{M}=6.6 \times 10^{-5} \mathrm{M}_{\odot}, \mathrm{T}=650 \mathrm{~K}\right)$ \\
\hline & & 736 & 0.5 & 830 & 340 & 18.0 & 0.1 & + cold comp. $\left(\mathrm{R}=5.4 \times 10^{16} \mathrm{~cm}, \mathrm{~T}=110 \mathrm{~K}\right)$ \\
\hline
\end{tabular}


Table A4 (continued)

\begin{tabular}{|c|c|c|c|c|c|c|c|c|}
\hline Object & Type & $\begin{array}{l}\text { Epoch } \\
\text { (days) }\end{array}$ & $\begin{array}{c}R_{B B} \\
\left(10^{16} \mathrm{~cm}\right)\end{array}$ & $\begin{array}{c}v_{B B} \\
\left(\mathrm{~km} \mathrm{~s}^{-1}\right)\end{array}$ & $\begin{array}{c}T_{\text {dust }} \\
(\mathrm{K})\end{array}$ & $\begin{array}{c}M_{\text {dust }} \\
\left(10^{-5} \mathrm{M}_{\odot}\right)\end{array}$ & $\begin{array}{c}L_{\text {dust }} \\
\left(10^{6} L_{\odot}\right)\end{array}$ & Notes \\
\hline & & 1125 & 0.4 & 1250 & 420 & 10.0 & 0.2 & + hot comp. $\left(\mathrm{R}=0.01 \times 10^{16} \mathrm{~cm}, \mathrm{~T}=6300 \mathrm{~K}\right)$ \\
\hline & & 1222 & 0.5 & 650 & 420 & 11.0 & 0.2 & + cold comp. $\left(\mathrm{R}=4.5 \times 10^{16} \mathrm{~cm}, \mathrm{~T}=120 \mathrm{~K}\right)$ \\
\hline \multirow[t]{2}{*}{ SN 2005ad } & $\mathrm{II}-\mathrm{P}$ & 198 & 0.3 & 1755 & 630 & 6.0 & 1.1 & \\
\hline & & 364 & 0.2 & 635 & 580 & 3.8 & 0.4 & \\
\hline \multirow[t]{4}{*}{ SN 2005af } & $\mathrm{II}-\mathrm{P}$ & 194 & 0.8 & 4770 & 510 & 43.6 & 2.5 & \\
\hline & & 399 & 0.4 & 1160 & 510 & 10.9 & 0.6 & Possible Si dust $\left(M=1.6 \times 10^{-5} M_{\odot}, T=640 K\right)$ \\
\hline & & 576 & 0.6 & 1205 & 340 & 64.5 & 0.4 & Possible Si dust $\left(M=2.6 \times 10^{-5} M_{\odot}, T=460 K\right)$ \\
\hline & & 772 & 0.8 & 1200 & 290 & 58.8 & 0.2 & Possible Si dust $\left(M=0.7 \times 10^{-5} M_{\odot}, T=430 K\right)$ \\
\hline SN 2005at & Ic & 569 & 2.7 & 5490 & 270 & $<640.0$ & $<1.4$ & + hot comp. $\left(\mathrm{R}=7.0 \times 10^{13} \mathrm{~cm}, \mathrm{~T}=10500 \mathrm{~K}\right)$ \\
\hline \multirow[t]{2}{*}{$S N 2005 c p$} & IIn & 1523 & 3.6 & 2730 & 550 & $<680$ & $<56.0$ & \\
\hline & & 2254 & 2.0 & 1040 & 730 & $<52.0$ & $<22.0$ & Only a two-point mid-IR SED \\
\hline \multirow[t]{3}{*}{ SN 2005gj } & $\mathrm{Ia}-\mathrm{CSM}$ & 139 & 3.3 & 27480 & 845 & 100 & 100 & \\
\hline & & 365 & 4.0 & 12680 & 845 & 200 & 150 & \\
\hline & & 510 & 6.8 & 15430 & 725 & 600 & 230 & \\
\hline SN 2005gn & IIn & 1479 & 1.5 & 1180 & 740 & 190 & 84.0 & Only a two-point mid-IR SED \\
\hline \multirow[t]{2}{*}{$S N 2005 i p$} & IIn & 948 & 4.8 & 5860 & 470 & $<4200$ & $<140$ & \\
\hline & & 2057 & 8.5 & 4790 & 510 & $<1900$ & $<100$ & Only a two-point mid-IR SED \\
\hline \multirow[t]{3}{*}{$S N 2006 b p$} & $I I-P$ & 401 & 4.5 & 12990 & 370 & $<480$ & $<4.7$ & + cold comp. $\left(R=24 \times 10^{16} \mathrm{~cm}, T=110 \mathrm{~K}\right)$ \\
\hline & & 628 & 5.5 & 10140 & 330 & $<1000$ & $<4.4$ & \\
\hline & & 767 & 5.1 & 7695 & 350 & $<690$ & $<4.6$ & + cold comp. $\left(R=42 \times 10^{16} \mathrm{~cm}, T=80 \mathrm{~K}\right)$ \\
\hline \multirow[t]{2}{*}{ SN 2006jc } & Ibn & 210 & 1.3 & 7160 & 600 & 59.5 & 8.6 & \\
\hline & & 412 & 1.5 & 4210 & 440 & 71.6 & 1.9 & \\
\hline$S N 2006 j d$ & IIn & 1149 & 6.3 & 6380 & 580 & $<3000$ & $<320$ & Only a two-point mid-IR SED \\
\hline
\end{tabular}


Table A4 (continued)

\begin{tabular}{|c|c|c|c|c|c|c|c|c|}
\hline Object & Type & $\begin{array}{l}\text { Epoch } \\
\text { (days) }\end{array}$ & $\begin{array}{c}R_{B B} \\
\left(10^{16} \mathrm{~cm}\right)\end{array}$ & $\begin{array}{c}v_{B B} \\
\left(\mathrm{~km} \mathrm{~s}^{-1}\right)\end{array}$ & $\begin{array}{c}T_{d u s t} \\
(\mathrm{~K})\end{array}$ & $\begin{array}{c}M_{d u s t} \\
\left(10^{-5} \mathrm{M}_{\odot}\right)\end{array}$ & $\begin{array}{c}L_{d u s t} \\
\left(10^{6} L_{\odot}\right)\end{array}$ & Notes \\
\hline \multirow{4}{*}{ SN 2006my } & \multirow{4}{*}{$\mathrm{II}-\mathrm{P}$} & 1714 & 11.4 & 7670 & 530 & $<4000$ & $<230$ & Only a two-point mid-IR SED \\
\hline & & 205 & 3.8 & 21455 & 280 & 4920 & 12.6 & + hot comp. $\left(\mathrm{R}=0.1 \times 10^{16} \mathrm{~cm}, \mathrm{~T}=3700 \mathrm{~K}\right)$ \\
\hline & & 342 & 3.7 & 12520 & 300 & 3074 & 11.1 & + cold comp. $\left(\mathrm{R}=33.5 \times 10^{16} \mathrm{~cm}, \mathrm{~T}=120 \mathrm{~K}\right)$ \\
\hline & & 559 & 4.2 & 8695 & 290 & 3960 & 12.1 & + cold comp. $\left(\mathrm{R}=76 \times 10^{16} \mathrm{~cm}, \mathrm{~T}=90 \mathrm{~K}\right)$ \\
\hline \multirow[t]{2}{*}{ SN 2006ov } & \multirow[t]{2}{*}{$\mathrm{II}-\mathrm{P}$} & 321 & 5.1 & 18390 & 280 & 2500 & 6.4 & \\
\hline & & 539 & 3.4 & 7300 & 310 & 976 & 4.1 & \\
\hline \multirow[t]{2}{*}{$S N 2006 q q$} & \multirow[t]{2}{*}{ IIn } & 1048 & 4.5 & 5010 & 500 & $<1600$ & $<77.0$ & Only a two-point mid-IR SED \\
\hline & & 1805 & 3.3 & 2120 & 770 & $<130$ & $<75.0$ & Only a two-point mid-IR SED \\
\hline \multirow[t]{4}{*}{ SN 2007it } & \multirow[t]{4}{*}{$\mathrm{II}-\mathrm{P}$} & 343 & 0.3 & 910 & 640 & 5.0 & 1.0 & \\
\hline & & 553 & 0.4 & 900 & 640 & 10.6 & 2.0 & \\
\hline & & 710 & 0.6 & 960 & 530 & 15.9 & 1.1 & \\
\hline & & 934 & 1.8 & 2180 & 350 & 133 & 1.0 & \\
\hline \multirow[t]{2}{*}{ SN 2007oc } & \multirow[t]{2}{*}{$\mathrm{II}-\mathrm{P}$} & 251 & 5.7 & 26285 & 340 & 310 & 9.8 & \\
\hline & & 415 & 4.9 & 13665 & 340 & 370 & 6.1 & \\
\hline \multirow[t]{2}{*}{ SN $200 \% r t$} & \multirow[t]{2}{*}{ IIn } & 780 & 5.4 & 8000 & 625 & $<2200$ & $<370$ & Only a two-point mid-IR SED \\
\hline & & 1304 & 8.5 & 7560 & 555 & $<2300$ & $<190$ & Only a two-point mid-IR SED \\
\hline SN 2008J & IIn & 593 & 3.3 & 6460 & 700 & 870 & 290 & Only a two-point mid-IR SED \\
\hline \multirow[t]{2}{*}{$S N 2008 c g$} & \multirow[t]{2}{*}{ IIn } & 474 & 3.4 & 8320 & 600 & $<1300$ & $<180$ & Only a two-point mid-IR SED \\
\hline & & 1226 & 3.0 & 2860 & 575 & $<310$ & $<32.0$ & Only a two-point mid-IR SED \\
\hline \multirow[t]{2}{*}{ SN 2008en } & \multirow[t]{2}{*}{ IIn } & 386 & 2.9 & 8790 & 680 & $<670$ & $<180$ & Only a two-point mid-IR SED \\
\hline & & 1131 & 5.7 & 5810 & 560 & $<680$ & $<62.0$ & Only a two-point mid-IR SED \\
\hline SN $2008 f q$ & II & 1168 & 0.4 & 420 & 600 & 9.5 & 1.3 & Only a two-point mid-IR SED \\
\hline SN $2008 \mathrm{gm}$ & IIn & 301 & 0.4 & 1460 & 720 & $<6.5$ & $<2.5$ & Only a two-point mid-IR SED \\
\hline
\end{tabular}

Table A4 continued on next page 
Table A4 (continued)

\begin{tabular}{|c|c|c|c|c|c|c|c|c|}
\hline Object & Type & $\begin{array}{l}\text { Epoch } \\
\text { (days) }\end{array}$ & $\begin{array}{c}R_{B B} \\
\left(10^{16} \mathrm{~cm}\right)\end{array}$ & $\begin{array}{c}v_{B B} \\
\left(\mathrm{~km} \mathrm{~s}^{-1}\right)\end{array}$ & $\begin{array}{c}T_{d u s t} \\
(\mathrm{~K})\end{array}$ & $\begin{array}{c}M_{d u s t} \\
\left(10^{-5} \mathrm{M}_{\odot}\right)\end{array}$ & $\begin{array}{c}L_{d u s t} \\
\left(10^{6} L_{\odot}\right)\end{array}$ & Notes \\
\hline & & 1035 & 0.5 & 530 & 1070 & $<1.6$ & $<6.4$ & Only a two-point mid-IR SED \\
\hline SN 2008ip & IIn & 438 & 0.8 & 2000 & 610 & $<42.0$ & $<5.9$ & Only a two-point mid-IR SED \\
\hline SN 2009E & II-P & 547 & 3.0 & 6330 & 340 & 5080 & 3.5 & Only a two-point mid-IR SED \\
\hline \multirow[t]{2}{*}{$S N 2009 H$} & $I I$ & 243 & 1.6 & 7670 & 600 & $<93.2$ & $<13.2$ & Only a two-point mid-IR SED \\
\hline & & 282 & 1.8 & 7350 & 580 & $<95.3$ & $<11.2$ & Only a two-point mid-IR SED \\
\hline \multirow[t]{2}{*}{ SN 2009af } & $I I$ & 199 & 2.4 & 14130 & 460 & $<255$ & $<8.5$ & Only a two-point mid-IR SED \\
\hline & & 229 & 2.5 & 12790 & 430 & $<295$ & $<6.8$ & Only a two-point mid-IR SED \\
\hline \multirow[t]{2}{*}{ SN 2009at } & $I I$ & 474 & 0.3 & 780 & 750 & $<5.1$ & $<2.5$ & Only a two-point mid-IR SED \\
\hline & & 505 & 0.3 & 760 & 730 & $<5.5$ & $<2.3$ & Only a two-point mid-IR SED \\
\hline \multirow[t]{3}{*}{ SN 2009ip } & IIn & 712 & 0.3 & 420 & 470 & $<3.8$ & $<0.1$ & Only a two-point mid-IR SED \\
\hline & & 1085 & 0.3 & 290 & 450 & $<3.8$ & $<0.1$ & Only a two-point mid-IR SED \\
\hline & & 1424 & 0.1 & 110 & 540 & $<0.9$ & $<0.1$ & Only a two-point mid-IR SED \\
\hline SN 2009jf & $\mathrm{Ib}$ & 100 & 1.5 & 17360 & 520 & $<98.5$ & $<6.4$ & 2 mid-IR point + hot comp. $\left(\mathrm{R}=0.09 \times 10^{16} \mathrm{~cm}, \mathrm{~T}=5760 \mathrm{~K}\right)$ \\
\hline SN 2009mk & IIb & 225 & 0.3 & 1290 & 860 & 3.5 & 3.8 & Only a two-point mid-IR SED \\
\hline \multirow[t]{2}{*}{ SN $2010 \mathrm{~F}$} & II & 207 & 3.0 & 16770 & 380 & 452.9 & 5.5 & Only a two-point mid-IR SED \\
\hline & & 378 & 1.0 & 3060 & 420 & 41.7 & 0.9 & Only a two-point mid-IR SED \\
\hline \multirow[t]{7}{*}{ SN 2010jl } & IIn & 67 & 3.1 & 53550 & 780 & 490 & 306 & Only a two-point mid-IR SED \\
\hline & & 229 & 3.7 & 18700 & 720 & 668 & 265 & Only a two-point mid-IR SED \\
\hline & & 440 & 3.8 & 10000 & 810 & 805 & 621 & Only a two-point mid-IR SED \\
\hline & & 596 & 4.1 & 7960 & 800 & 872 & 629 & Only a two-point mid-IR SED \\
\hline & & 819 & 4.6 & 6500 & 750 & 1090 & 544 & Only a two-point mid-IR SED \\
\hline & & 969 & 5.7 & 6810 & 720 & 1620 & 641 & Only a two-point mid-IR SED \\
\hline & & 1343 & 6.6 & 5690 & 620 & 2094 & 356 & Only a two-point mid-IR SED \\
\hline
\end{tabular}


Table A4 (continued)

\begin{tabular}{|c|c|c|c|c|c|c|c|c|}
\hline Object & Type & $\begin{array}{l}\text { Epoch } \\
\text { (days) }\end{array}$ & $\begin{array}{c}R_{B B} \\
\left(10^{16} \mathrm{~cm}\right)\end{array}$ & $\begin{array}{c}v_{B B} \\
\left(\mathrm{~km} \mathrm{~s}^{-1}\right)\end{array}$ & $\begin{array}{c}T_{\text {dust }} \\
(\mathrm{K})\end{array}$ & $\begin{array}{c}M_{d u s t} \\
\left(10^{-5} \mathrm{M}_{\odot}\right)\end{array}$ & $\begin{array}{c}L_{\text {dust }} \\
\left(10^{6} L_{\odot}\right)\end{array}$ & Notes \\
\hline & & 1725 & 7.5 & 5030 & 540 & 2674 & 211 & Only a two-point mid-IR SED \\
\hline \multirow[t]{2}{*}{ SN $2010 m c$} & IIn & 1386 & 1.0 & 840 & 760 & $<50.4$ & $<27.1$ & Only a two-point mid-IR SED \\
\hline & & 1735 & 1.0 & 650 & 750 & $<49.0$ & $<24.4$ & Only a two-point mid-IR SED \\
\hline \multirow[t]{7}{*}{ SN 2011dh } & IIb & 250 & 0.7 & 3240 & 520 & 10.0 & 0.8 & Only a two-point mid-IR SED \\
\hline & & 280 & 1.6 & 6610 & 410 & 90.0 & 1.3 & Only a two-point mid-IR SED \\
\hline & & 312 & 0.7 & 2600 & 450 & 20.0 & 0.4 & Only a two-point mid-IR SED \\
\hline & & 390 & 0.6 & 1780 & 390 & 10.0 & 0.2 & Only a two-point mid-IR SED \\
\hline & & 422 & 0.4 & 1100 & 420 & 5.0 & 0.01 & Only a two-point mid-IR SED \\
\hline & & 454 & 0.3 & 870 & 420 & 4.0 & 0.01 & Only a two-point mid-IR SED \\
\hline & & 623 & 0.1 & 190 & 510 & 0.3 & 0.002 & Only a two-point mid-IR SED \\
\hline SN 2011dq & II & 1259 & 0.3 & 260 & 1080 & 6.0 & 25.0 & Only a two-point mid-IR SED \\
\hline SN 2011fh & IIn & 586 & 1.7 & 3280 & 360 & 439 & 4.0 & Only a two-point mid-IR SED \\
\hline SN 2011ir & IIn & 832 & 0.9 & 1180 & 610 & $<35.1$ & $<5.4$ & Only a two-point mid-IR SED \\
\hline \multirow[t]{6}{*}{ SN 2011ja } & $\mathrm{II}-\mathrm{P}$ & 106 & 1.0 & 10920 & 610 & 18.0 & 2.6 & Only a two-point mid-IR SED \\
\hline & & 488 & 1.1 & 2610 & 590 & 23.0 & 2.7 & Only a two-point mid-IR SED \\
\hline & & 639 & 1.1 & 1990 & 530 & 26.0 & 1.6 & Only a two-point mid-IR SED \\
\hline & & 859 & 1.2 & 1620 & 440 & 45.0 & 1.0 & Only a two-point mid-IR SED \\
\hline & & 881 & 1.2 & 1580 & 430 & 43.0 & 0.9 & Only a two-point mid-IR SED \\
\hline & & 1007 & 1.4 & 1610 & 380 & 76.0 & 0.7 & Only a two-point mid-IR SED \\
\hline \multirow[t]{2}{*}{$P T F 11 k x$} & $I a-C S M$ & 1237 & 5.0 & 4680 & 590 & $<400$ & $<57.0$ & Only a two-point mid-IR SED \\
\hline & & 1818 & 5.0 & 3180 & 580 & $<200$ & $<26.0$ & Only a two-point mid-IR SED \\
\hline \multirow[t]{2}{*}{ PTF11qcj } & Ic & 174 & 1.0 & 6650 & 620 & 52.4 & 8.9 & Only a two-point mid-IR SED \\
\hline & & 263 & 0.9 & 3960 & 560 & 37.5 & 3.6 & Only a two-point mid-IR SED \\
\hline
\end{tabular}

Table A4 continued on next page 
Table A4 (continued)

\begin{tabular}{|c|c|c|c|c|c|c|c|c|}
\hline Object & Type & $\begin{array}{l}\text { Epoch } \\
\text { (days) }\end{array}$ & $\begin{array}{c}R_{B B} \\
\left(10^{16} \mathrm{~cm}\right)\end{array}$ & $\begin{array}{c}v_{B B} \\
\left(\mathrm{~km} \mathrm{~s}^{-1}\right)\end{array}$ & $\begin{array}{c}T_{\text {dust }} \\
(\mathrm{K})\end{array}$ & $\begin{array}{c}M_{d u s t} \\
\left(10^{-5} \mathrm{M}_{\odot}\right)\end{array}$ & $\begin{array}{c}L_{d u s t} \\
\left(10^{6} L_{\odot}\right)\end{array}$ & Notes \\
\hline \multirow[t]{3}{*}{ SN 2012ca } & $I a-C S M$ & 404 & 3.3 & 9450 & 680 & $<537$ & $<154$ & Only a two-point mid-IR SED \\
\hline & & 577 & 5.3 & 10630 & 610 & $<1302$ & $<202$ & Only a two-point mid-IR SED \\
\hline & & 778 & 10.4 & 15470 & 450 & $<4445$ & $<131$ & Only a two-point mid-IR SED \\
\hline \multirow[t]{2}{*}{ SN $2012 f h$} & Ic & 214 & 1.4 & 7460 & 500 & 87.5 & 4.5 & Only a two-point mid-IR SED \\
\hline & & 247 & 1.4 & 6560 & 470 & 89.0 & 3.3 & \\
\hline \multirow[t]{2}{*}{$S N 2013 L$} & IIn & 630 & 2.6 & 4780 & 720 & $<341$ & $<124$ & Only a two-point mid-IR SED \\
\hline & & 850 & 3.4 & 4630 & 650 & $<573$ & $<127$ & Only a two-point mid-IR SED \\
\hline SN 2013ai & II & 705 & 0.6 & 820 & 400 & 13.0 & 0.2 & Only a two-point mid-IR SED \\
\hline \multirow[t]{3}{*}{ SN 2013am } & II & 343 & 0.4 & 1490 & 630 & 3.1 & 0.6 & Only a two-point mid-IR SED \\
\hline & & 369 & 0.9 & 2760 & 480 & 21.0 & 0.8 & Only a two-point mid-IR SED \\
\hline & & 493 & 0.5 & 1130 & 510 & 5.6 & 0.3 & Only a two-point mid-IR SED \\
\hline SN 2013bu & II & 287 & 0.7 & 2700 & 550 & 11.0 & 0.9 & Only a two-point mid-IR SED \\
\hline \multirow[t]{2}{*}{$S N 2013 c j$} & IIn & 394 & 2.9 & 8550 & 880 & $<490$ & $<614$ & Only a two-point mid-IR SED \\
\hline & & 747 & 4.2 & 6570 & 780 & $<967$ & $<606$ & Only a two-point mid-IR SED \\
\hline \multirow[t]{5}{*}{ SN 2013df } & IIb & 268 & 1.0 & 4320 & 480 & 28.0 & 1.0 & Only a two-point mid-IR SED \\
\hline & & 294 & 1.0 & 3940 & 450 & 32.0 & 0.8 & Only a two-point mid-IR SED \\
\hline & & 423 & 1.3 & 3560 & 340 & 78.0 & 0.4 & Only a two-point mid-IR SED \\
\hline & & 459 & 0.3 & 760 & 520 & 2.6 & 0.1 & Only a two-point mid-IR SED \\
\hline & & 824 & 0.1 & 140 & 740 & 0.1 & 0.06 & Only a two-point mid-IR SED \\
\hline \multirow[t]{5}{*}{ SN $2013 \mathrm{dk}$} & Ic & 268 & 0.8 & 3450 & 620 & 12.0 & 1.9 & Only a two-point mid-IR SED \\
\hline & & 269 & 1.0 & 4320 & 560 & 21.0 & 1.9 & Only a two-point mid-IR SED \\
\hline & & 292 & 0.8 & 3170 & 570 & 14.0 & 1.4 & Only a two-point mid-IR SED \\
\hline & & 298 & 0.7 & 2720 & 640 & 7.4 & 1.4 & Only a two-point mid-IR SED \\
\hline & & 428 & 0.4 & 1080 & 660 & 2.6 & 0.6 & Only a two-point mid-IR SED \\
\hline
\end{tabular}


Table A4 (continued)

\begin{tabular}{|c|c|c|c|c|c|c|c|c|}
\hline Object & Type & $\begin{array}{l}\text { Epoch } \\
\text { (days) }\end{array}$ & $\begin{array}{c}R_{B B} \\
\left(10^{16} \mathrm{~cm}\right)\end{array}$ & $\begin{array}{c}v_{B B} \\
\left(\mathrm{~km} \mathrm{~s}^{-1}\right)\end{array}$ & $\begin{array}{c}T_{d u s t} \\
(\mathrm{~K})\end{array}$ & $\begin{array}{c}M_{d u s t} \\
\left(10^{-5} \mathrm{M}_{\odot}\right)\end{array}$ & $\begin{array}{c}L_{d u s t} \\
\left(10^{6} L_{\odot}\right)\end{array}$ & Notes \\
\hline \multirow[t]{2}{*}{$S N 2013 d n$} & $I a-C S M$ & 463 & 3.7 & 9250 & 670 & $<645$ & $<170$ & Only a two-point mid-IR SED \\
\hline & & 828 & 2.3 & 3215 & 660 & $<253$ & $<61.0$ & Only a two-point mid-IR SED \\
\hline \multirow[t]{3}{*}{ SN2013ee } & II & 191 & 3.6 & 22050 & 350 & 690 & 5.4 & Only a two-point mid-IR SED \\
\hline & & 220 & 1.6 & 8470 & 430 & 118 & 2.7 & Only a two-point mid-IR SED \\
\hline & & 341 & 0.6 & 1870 & 520 & 14.8 & 1.0 & Only a two-point mid-IR SED \\
\hline \multirow[t]{3}{*}{ SN 2013ej } & $\mathrm{II}-\mathrm{P} / \mathrm{L}$ & 238 & 1.7 & 8300 & 490 & 62.0 & 2.9 & Only a two-point mid-IR SED \\
\hline & & 261 & 1.6 & 7100 & 465 & 72.0 & 2.2 & Only a two-point mid-IR SED \\
\hline & & 439 & 1.2 & 3160 & 360 & 64.0 & 0.4 & Only a two-point mid-IR SED \\
\hline SN 2013ff & Ic & 147 & 2.9 & 22830 & 420 & 419 & 8.6 & Only a two-point mid-IR SED \\
\hline \multirow[t]{8}{*}{ SN $2014 \mathrm{C}$} & $\mathrm{Ib}$ & 243 & 2.1 & 10000 & 620 & 75.0 & 13.0 & Only a two-point mid-IR SED \\
\hline & & 274 & 2.2 & 9290 & 640 & 74.0 & 14.0 & Only a two-point mid-IR SED \\
\hline & & 622 & 3.2 & 5950 & 570 & 200 & 20.0 & Only a two-point mid-IR SED \\
\hline & & 628 & 2.8 & 5160 & 590 & 150 & 18.0 & Only a two-point mid-IR SED \\
\hline & & 642 & 3.7 & 6670 & 540 & 290 & 20.0 & Only a two-point mid-IR SED \\
\hline & & 772 & 3.6 & 5400 & 520 & 290 & 17.0 & Only a two-point mid-IR SED \\
\hline & & 780 & 3.6 & 5340 & 510 & 320 & 17.0 & Only a two-point mid-IR SED \\
\hline & & 801 & 3.8 & 5490 & 500 & 360 & 17.0 & Only a two-point mid-IR SED \\
\hline SN $2014 \mathrm{G}$ & II-L & 561 & 1.0 & 2040 & 460 & 42.4 & 1.4 & Only a two-point mid-IR SED \\
\hline SN 2014L & Ic & 206 & 1.6 & 8990 & 480 & 64.0 & 2.4 & Only a two-point mid-IR SED \\
\hline \multirow[t]{5}{*}{ SN 2014bi } & $\mathrm{II}-\mathrm{P}$ & 264 & 8.9 & 39020 & 240 & 7200 & 4.9 & Only a two-point mid-IR SED \\
\hline & & 268 & 5.6 & 24180 & 260 & 2500 & 2.7 & Only a two-point mid-IR SED \\
\hline & & 272 & 5.5 & 23400 & 260 & 2600 & 2.6 & Only a two-point mid-IR SED \\
\hline & & 293 & 3.3 & 13040 & 285 & 730 & 1.3 & Only a two-point mid-IR SED \\
\hline & & 445 & 0.5 & 1300 & 430 & 8.3 & 0.2 & Only a two-point mid-IR SED \\
\hline
\end{tabular}

Table A4 continued on next page 
Table A4 (continued)

\begin{tabular}{cccccccc|c}
\hline \hline Object & Type & $\begin{array}{c}\text { Epoch } \\
\text { (days) }\end{array}$ & $\begin{array}{c}R_{B B} \\
\left(10^{16} \mathrm{~cm}\right)\end{array}$ & $\begin{array}{c}v_{B B} \\
\left(\mathrm{~km} \mathrm{~s}^{-1}\right)\end{array}$ & $\begin{array}{c}T_{\text {dust }} \\
(\mathrm{K})\end{array}$ & $\begin{array}{c}M_{\text {dust }} \\
\left(10^{-5} \mathrm{M}_{\odot}\right)\end{array}$ & $\begin{array}{c}L_{\text {dust }} \\
\left(10^{6} L_{\odot}\right)\end{array}$ \\
\hline SN 2014dt & Iax & 309 & 0.3 & 1120 & 710 & 1.3 & 0.5 & Only a two-point mid-IR SED \\
& & 317 & 0.3 & 1100 & 680 & 1.8 & 0.5 & Only a two-point mid-IR SED \\
& & 0.3 & 1030 & 770 & 1.3 & 0.8 & Only a two-point mid-IR SED \\
\hline
\end{tabular}


B. COMPARISON OF SINGLE-EPOCH SPITZER DETECTIONS WITH PRE-EXPLOSION 2MASS JHK DATA 


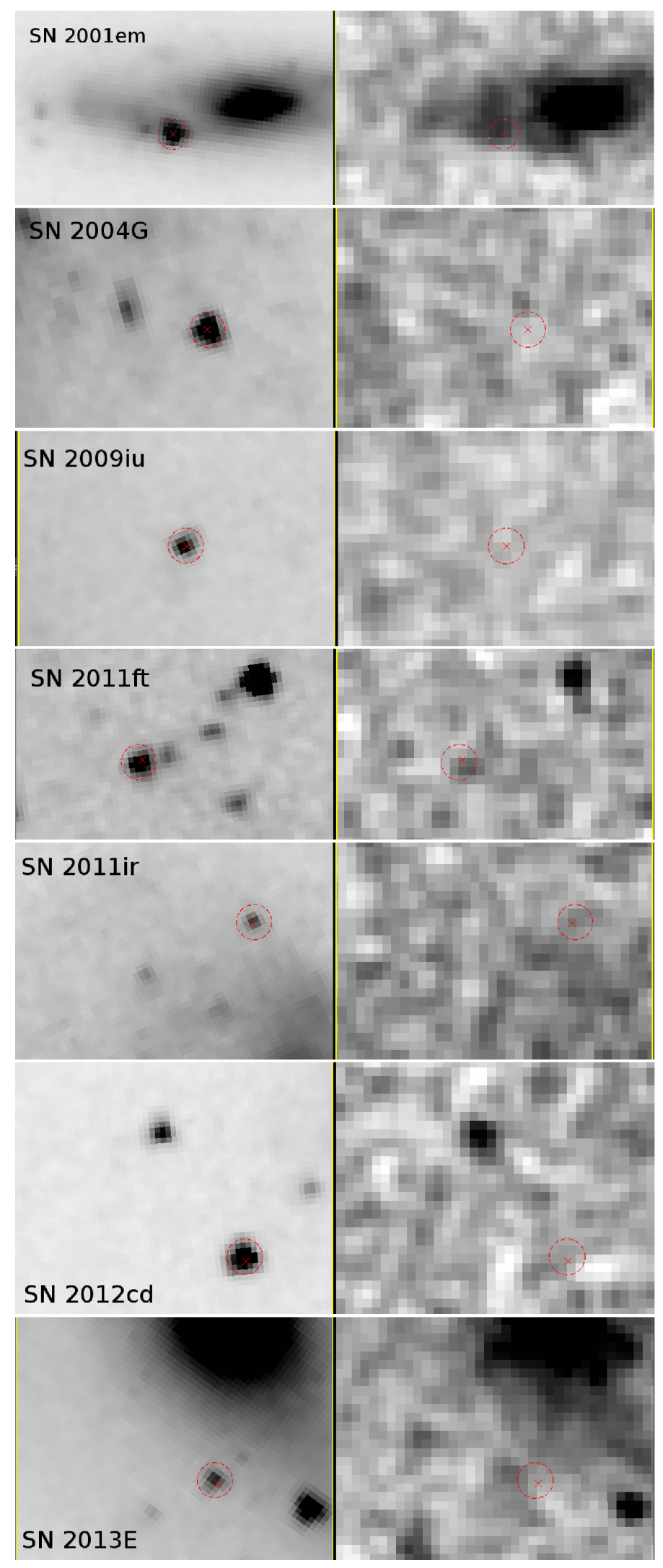

Figure B1. Comparison of single-epoch Spitzer/IRAC 3.6 micron images (left) of SNe (classified as positive detections) with pre-explosion 2MASS $\mathrm{K}_{s}$ images (right). Colors are inverted for better visibility. Red crosses denote the SN coordinates adopted from sources given in the Open Supernova Catalog, while red circles with radii of $2^{\prime \prime}$ show the typical PSF FWHM of point sources on IRAC images centered to the photometric centers of the mid-IR points sources. 
Table B1. Uncertainties of absolute coordinates ( $\sigma \mathrm{RA}, \sigma \mathrm{DEC})$, agreement of these coordinates and that of the photometric centers of mid-IR point sources $(\Delta \mathrm{XY})$, and pre-explosion 2 MASS $\mathrm{JHK}_{s}$ magnitudes (estimating a general \pm 0.4 magnitude error) regarding positively detected SNe based on single-epoch Spitzer/IRAC imaging.

\begin{tabular}{c|ccc|c|ccc}
\hline \hline Object & $\sigma R A$ & $\sigma$ DEC & Ref. & $\Delta$ XY & J (pre-exp.) & H (pre-exp.) & $\mathrm{K}_{s}$ (pre-exp.) \\
\hline SN 2001em & $<0.001^{\prime \prime}$ & $<0.001^{\prime \prime}$ & 1 & $<0.6^{\prime \prime}$ & $18.38(0.40)$ & $17.16(0.40)$ & $16.58(0.40)$ \\
SN 2004G & unknown & unknown & - & $<0.6^{\prime \prime}$ & $17.05(0.40)$ & $16.66(0.40)$ & $16.15(0.40)$ \\
SN 2009iu & $<0.1^{\prime \prime}$ & $<0.1^{\prime \prime}$ & 2 & $<0.6^{\prime \prime}$ & $18.15(0.40)$ & $18.50(0.40)$ & $19.54(0.40)$ \\
SN 2011ft & unknown & unknown & - & $<1.2^{\prime \prime}$ & $20.60(0.40)$ & $16.95(0.40)$ & $17.33(0.40)$ \\
SN 2011ir & unknown & unknown & - & $<1.2^{\prime \prime}$ & $19.25(0.40)$ & $20.03(0.40)$ & $17.78(0.40)$ \\
SN 2012cd & unknown & unknown & - & $<1.2^{\prime \prime}$ & $20.03(0.40)$ & $20.29(0.40)$ & $19.33(0.40)$ \\
SN 2013E & unknown & unknown & - & $<1.2^{\prime \prime}$ & $20.06(0.40)$ & $20.03(0.40)$ & $20.23(0.40)$ \\
\hline
\end{tabular}

Note-References: (1) Bietenholz \& Bartel (2005); (2) Maza et al. (2009) 

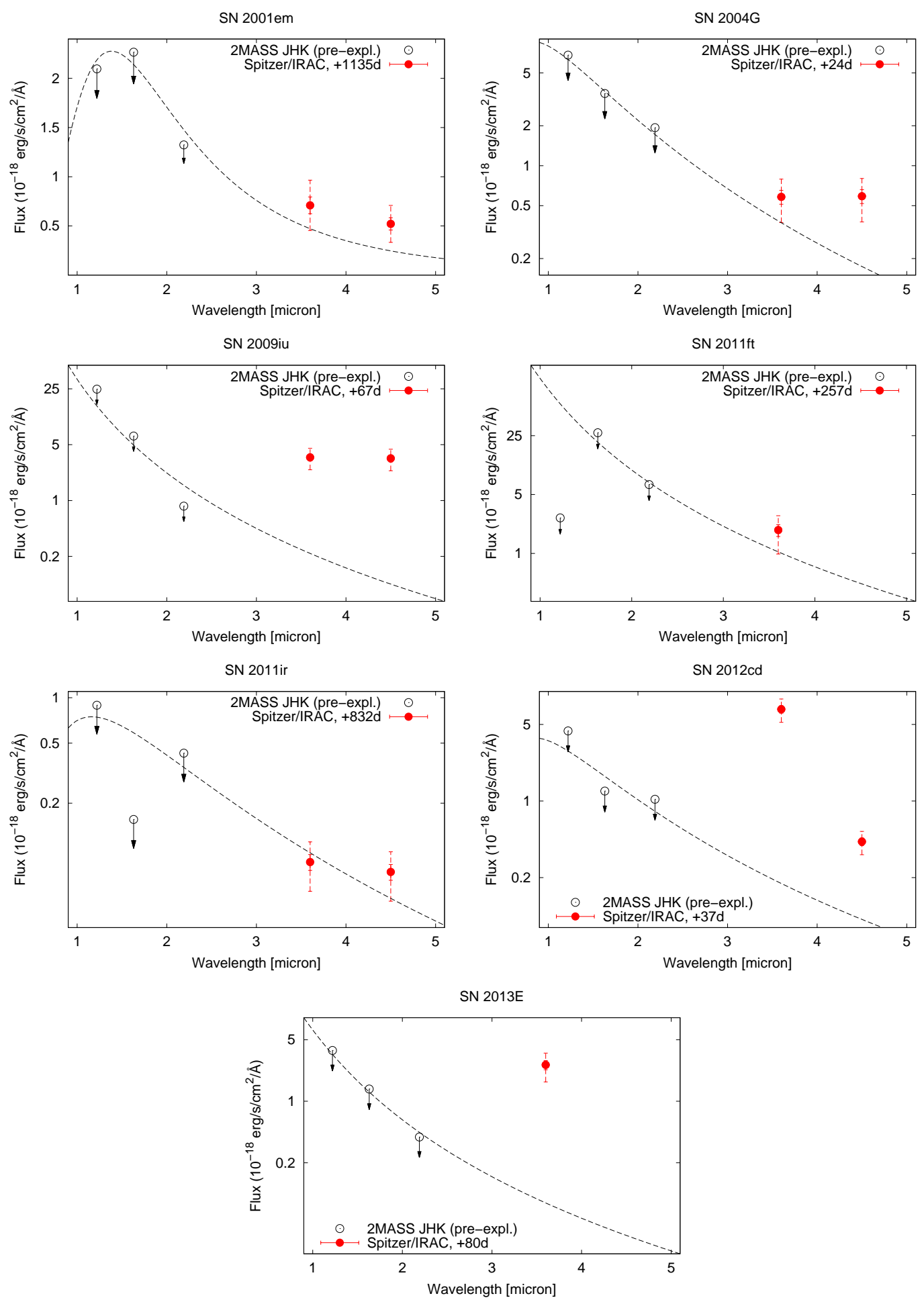

Figure B2. Comparison of pre-explosion 2MASS $\mathrm{JHK}_{s}$ (black open circles) and postexplosion mid-IR fluxes (red filled circles) in the cases of single-epoch Spitzer/IRAC SN observations classified as positive detections. Simple blackbodies are fitted to upper limits of $\mathrm{JHK}_{s}$ fluxes in order to see whether there can be any 'real' mid-IR excess at post-explosion Spitzer images or not (see text for details). In the cases of IRAC fluxes, solid and dashed errorbars denote $1-\sigma$ and $3-\sigma$ photometric errors, respectively. 

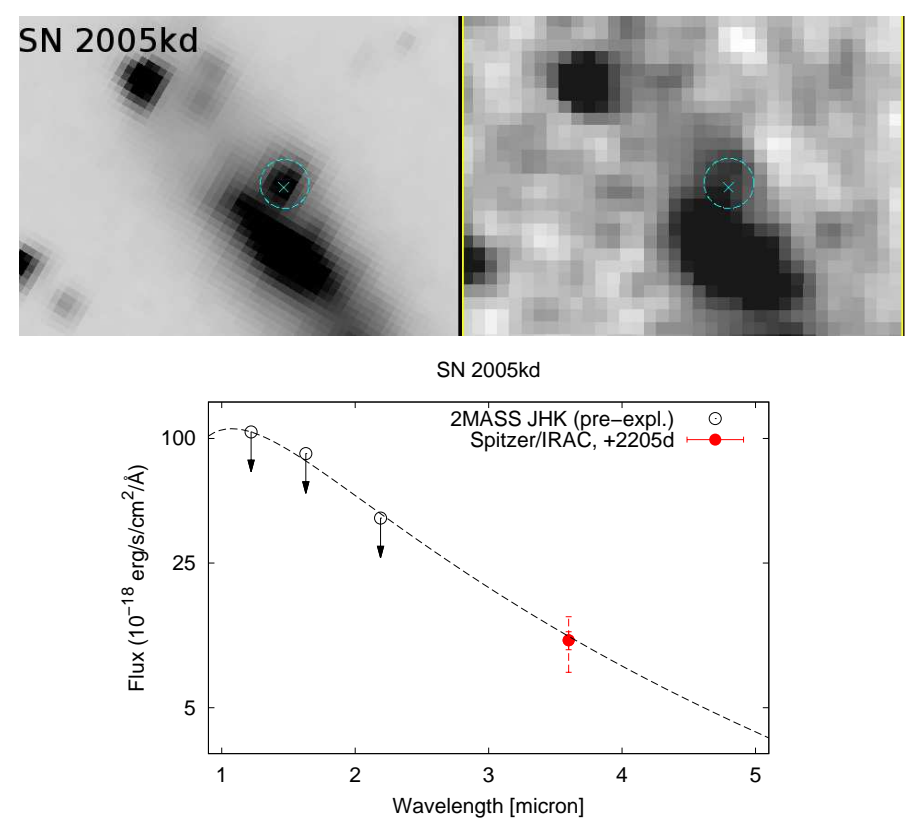

Figure B3. SN 2005kd: an example of single-epoch Spitzer/IRAC supernova observations classified as negative detections. While one can see a mid-IR source at the position of the $\mathrm{SN}$, some flux can be also detect on the pre-explosion 2MASS $\mathrm{K}_{s}$ image. Doing the final step of checking, no significant excess could be revealed at the $3.6 \mu \mathrm{m}$ channel (the field was not observed in any other IRAC channels). 